Aus der Klinik für Präventive Zahnmedizin, Parodontologie und Kariologie (Univ.-Prof. Dr. med. dent. Annette Wiegand) im Zentrum Zahn-, Mund- und Kieferheilkunde der Medizinischen Fakultät der Universität Göttingen

\title{
Vergleich der Effektivität aktivierter endodontischer Spültechniken in der Entfernung von Debris und smear layer von Wurzelkanalwänden
}

\author{
INAUGURAL-DISSERTATION \\ zur Erlangung des Doktorgrades \\ für Zahnheilkunde \\ der Medizinischen Fakultät der \\ Georg-August-Universität zu Göttingen
}

vorgelegt von

Michael Johannes Meinel

aus

Kassel

Göttingen 2019 
Dekan: $\quad$ Prof. Dr. rer. nat. H. K. Kroemer

Referent/in: Prof. Dr. M. Hülsmann

Ko-Referent/in:

Drittreferent/in:

Datum der mündlichen Prüfung: 
Hiermit erkläre ich, die Dissertation mit dem Titel "Vergleich der Effektivität aktivierter endodontischer Spültechniken in der Entfernung von Debris und smear layer von Wurzelkanalwänden" eigenständig angefertigt und keine anderen als die von mir angegebenen Quellen und Hilfsmittel verwendet zu haben.

Göttingen, den 


\section{Inhaltsverzeichnis}

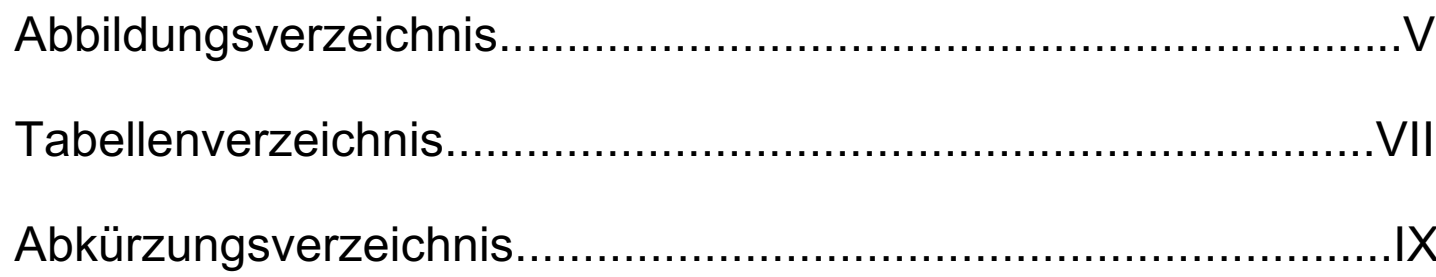

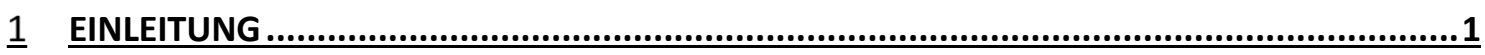

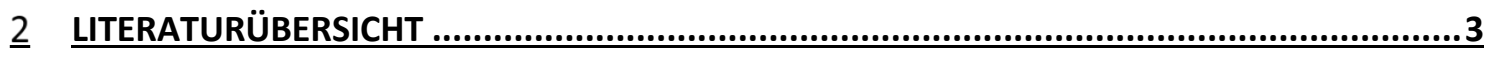

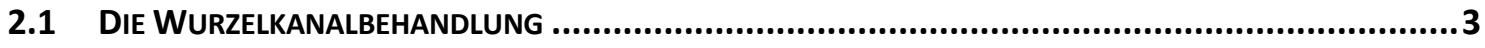

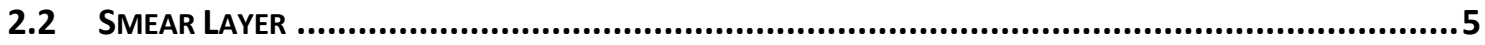

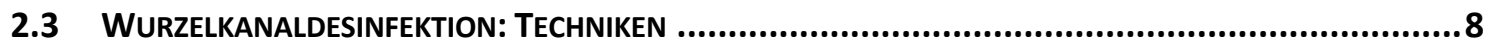

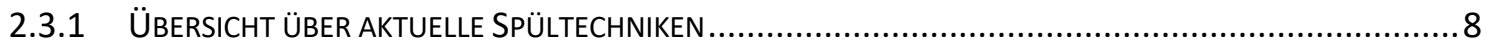

2.3.2 IN DIESER STUDIE VERWENDETE SPÜLTECHNIKEN .....................................................

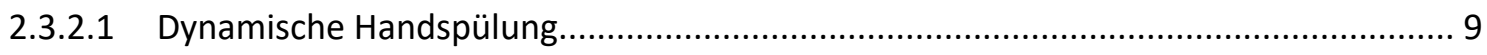

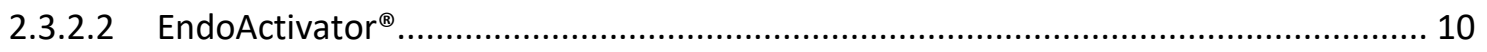

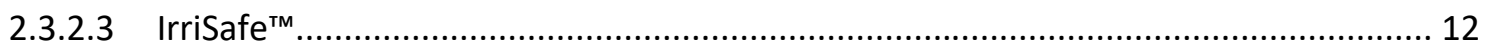

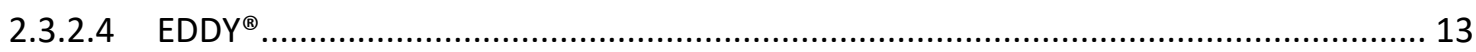

2.4 WURZELKANALDESINFEKTION: SPÜLLÖSUNGEN ...................................................... 15

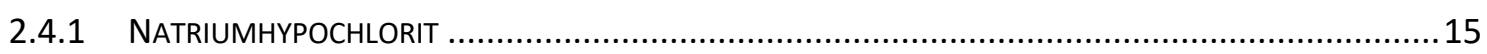

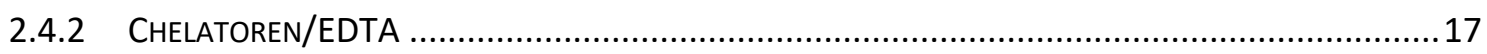

$\underline{3}$ ZIELE DER STUDIE.................................................................................20

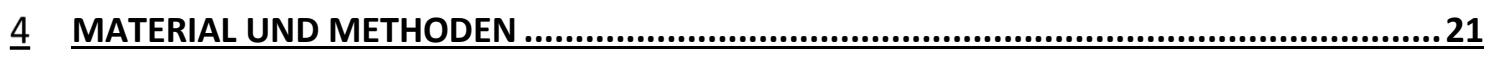

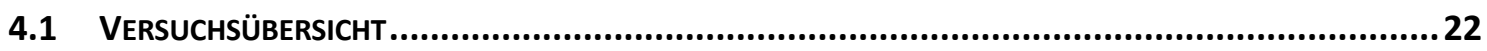

4.2 AUSWAHL DER ZÄHNE UND VORBEREITENDE MAßNAHMEN.............................................. 23

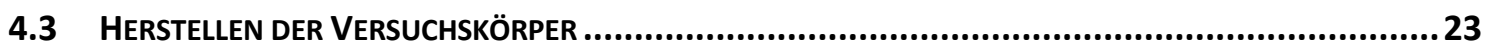

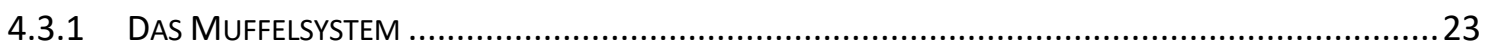




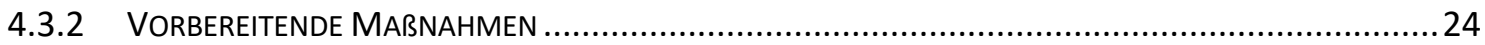

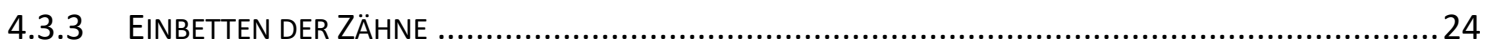

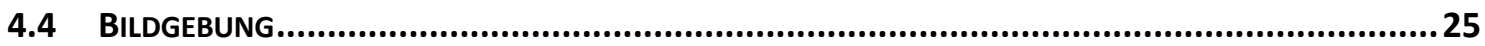

4.4.1 BERECHNUNG VON KRÜMMUNGSWINKEL UND -RADIUS DER WURZELKANÄLE ...............................25

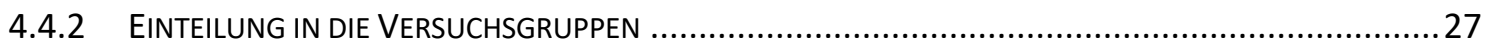

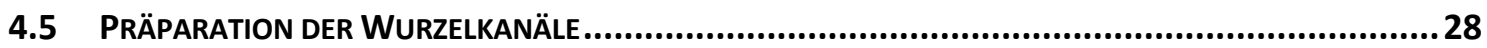

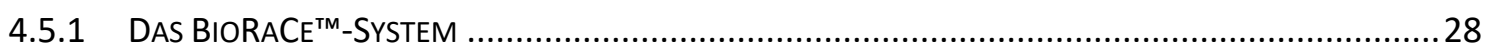

4.5.2 Die STANDARDISIERTE PRÄPARATION MIT DEM BIORACE ${ }^{\text {TM }}$ SYSTEM .............................................29

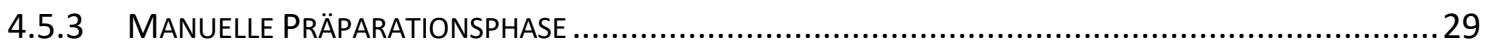

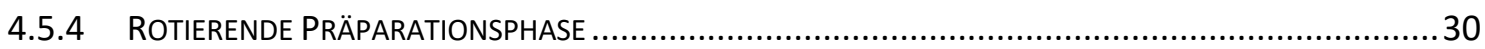

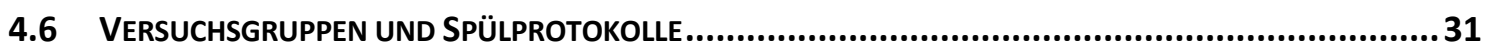

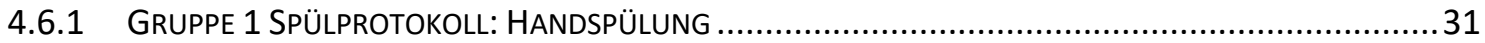

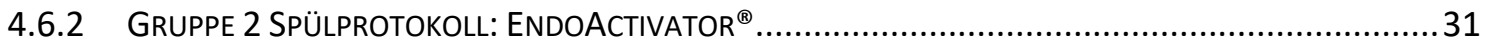

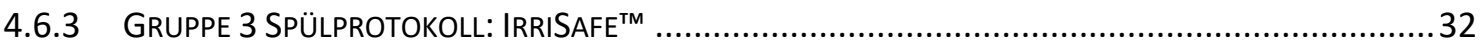

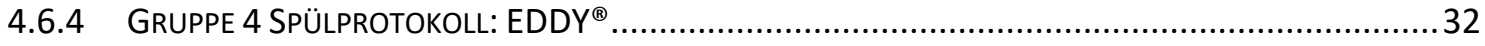

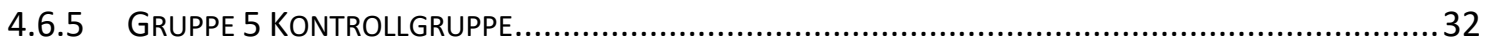

4.7 HeRSTELLUNG DER PROBEN für dAS RASTERELEKTRONENMIKROSKOP......................................33

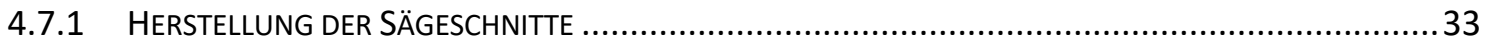

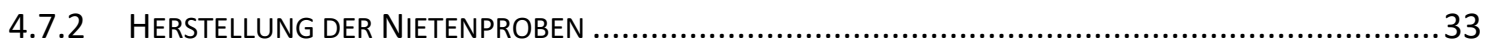

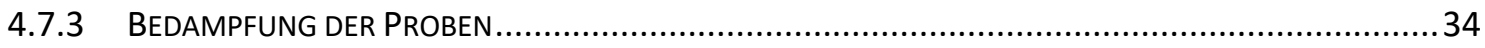

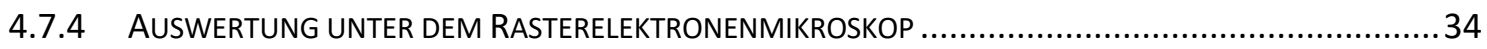

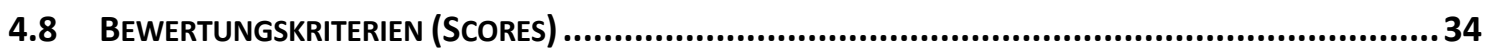

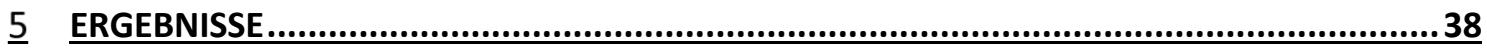

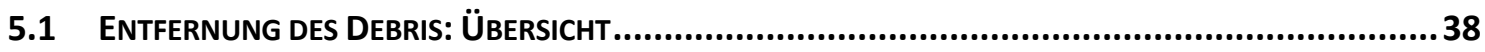

5.1.1 ENTFERNUNG DES DEBRIS IM KORONALEN WURZELKANALABSCHNITT ..........................................

5.1.2 ENTFERNUNG DES DEBRIS IM APIKALEN WURZELKANALABSCHNITT ............................................ 41

5.1.3 VERGLEICH DER KORONALEN UND APIKALEN REINIGUNGSWIRKUNG FÜR DEBRIS .............................42

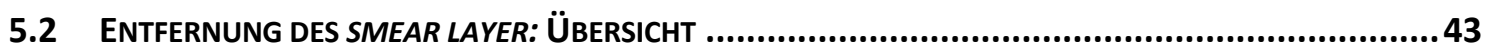

5.2.1 ENTFERNUNG DES SMEAR LAYER IM KORONALEN WURZELKANALABSCHNITT ..................................45

5.2.2 ENTFERNUNG DES SMEAR LAYER IM APIKALEN WURZELKANALABSCHNITT.....................................

5.2.3 ENTFERNUNG DES SMEAR LAYER IM KORONALEN UND APIKALEN KANALANTEIL ...............................48

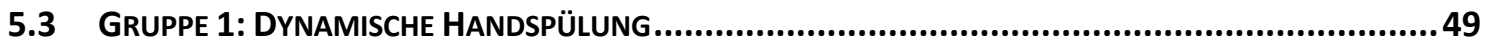

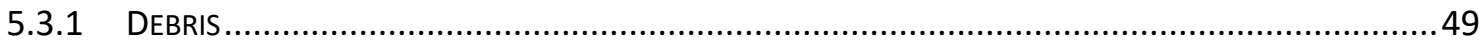

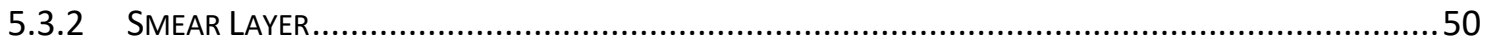




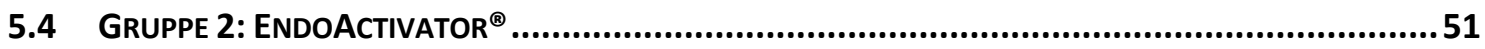

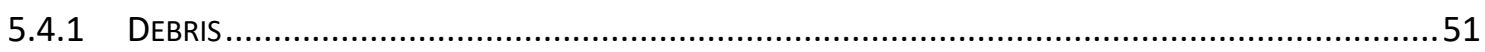

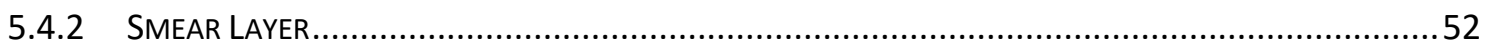

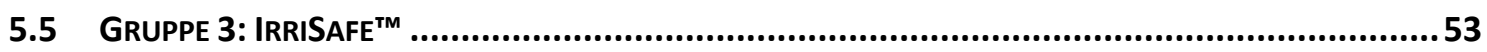

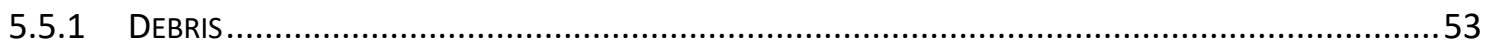

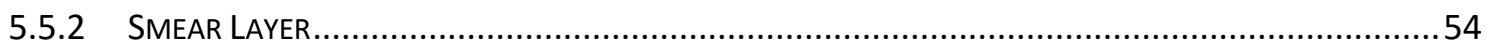

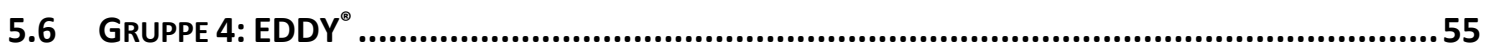

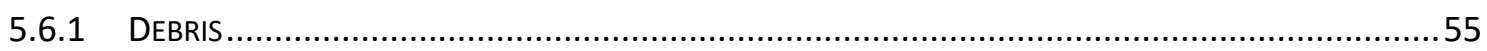

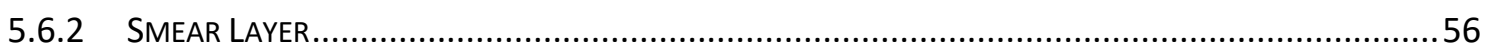

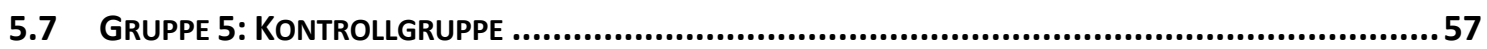

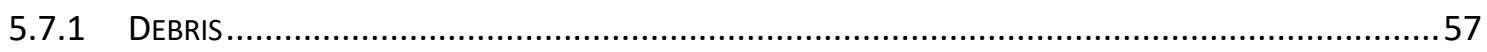

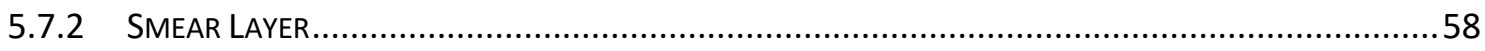

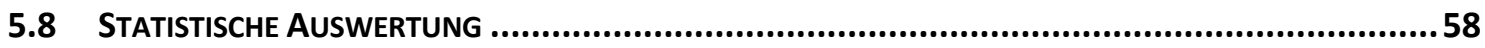

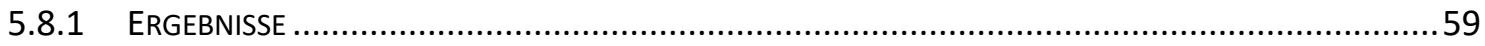

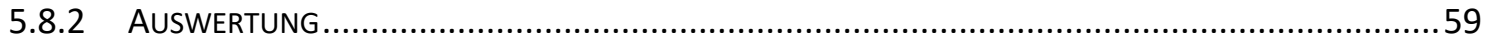

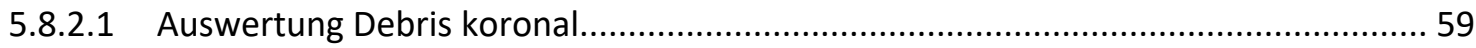

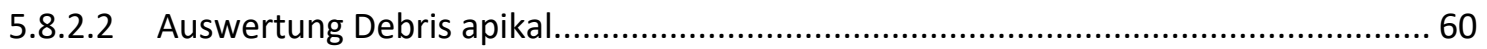

5.8.2.3 Auswertung smear layer koronal............................................................ 62

5.8.2.4 Auswertung smear layer apikal.............................................................. 63

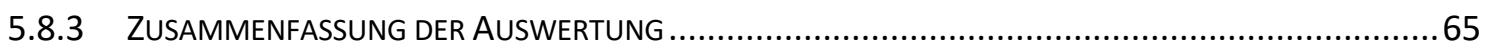

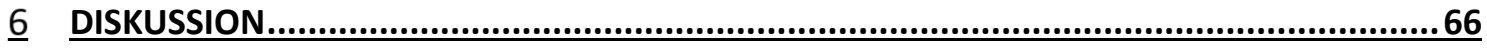

6.1 DisKUSSION VON MATERIAL UND METHOdEN...........................................................66

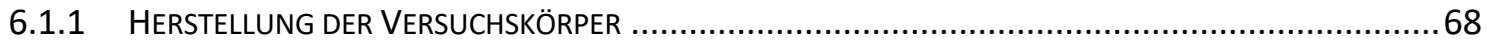

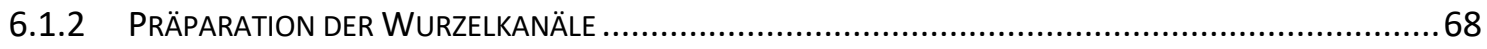

6.1.3 HERSTELLUNG DER PROBEN FÜR DAS RASTERELEKTRONENMIKROSKOP .................................69

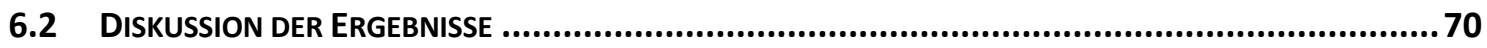

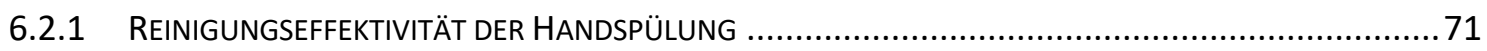

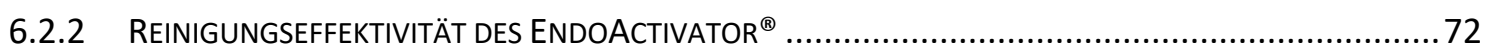

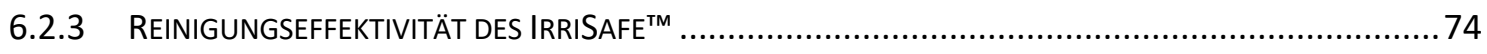

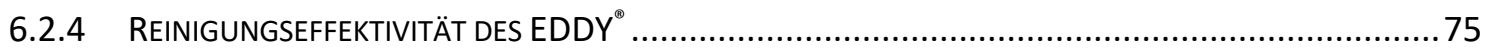

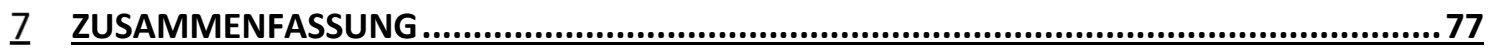

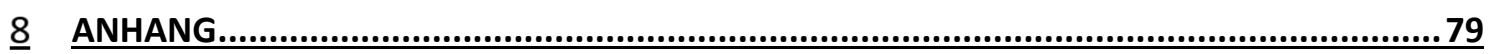

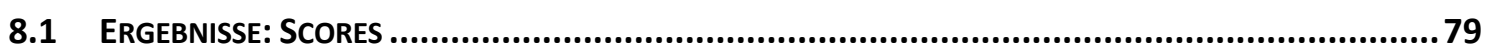




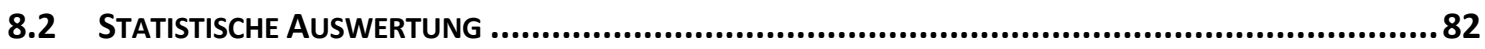

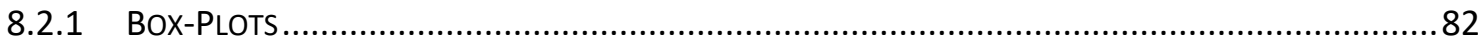

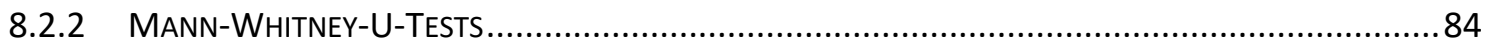

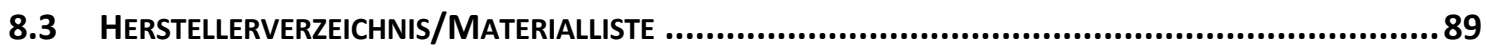

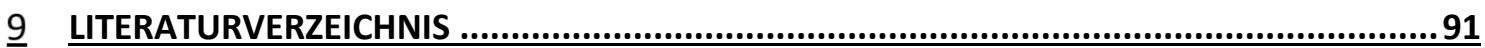


Abbildungsverzeichnis

ABBILDUNG 1: VERSUCHSÜBERSICHT 22

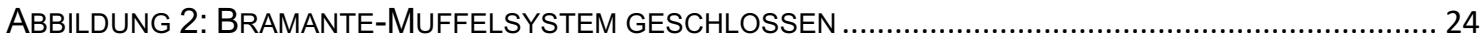

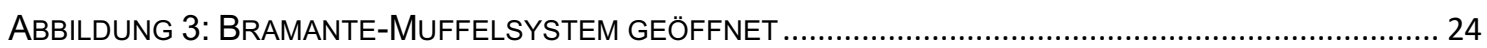

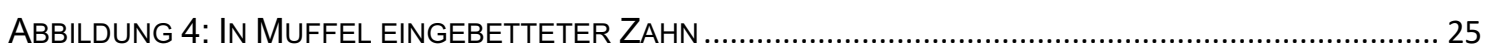

ABBILDUNG 5: BESTIMMUNG DES KRÜMMUNGSWINKELS NACH SCHNEIDER ........................................... 26

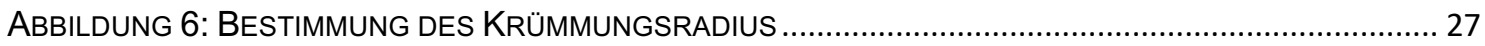

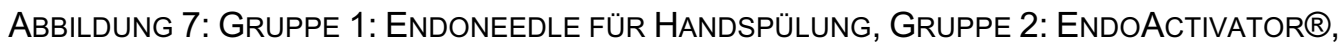

GRUPPE 3: IRRISAFE ${ }^{\text {TM }}$, GRUPPE 4: EDDY® ............................................................. 31

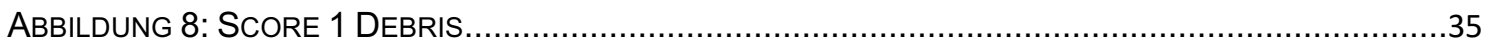

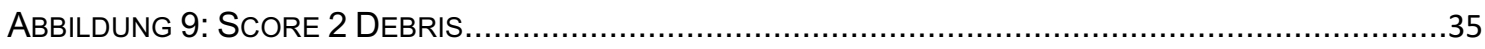

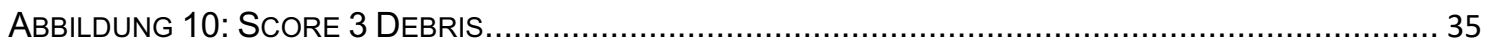

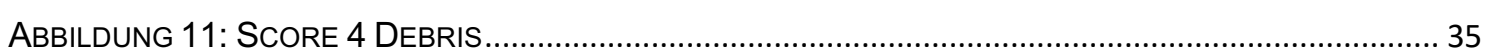

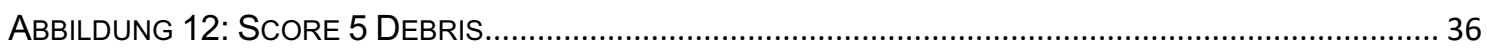

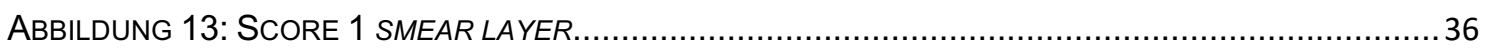

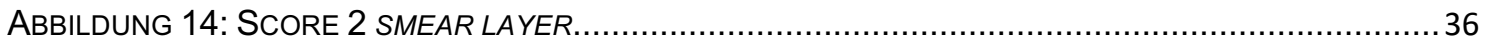

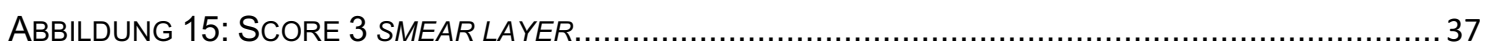

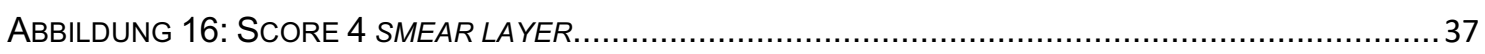

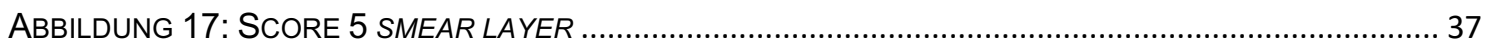

ABbildung 18: VeRTEILUNG deR SCORES FÜR DEBRIS NACH GRUPPEN (1 HANDSPÜLUNG, 2

ENDOACTIVATOR®, 3 IRRISAFE ${ }^{\mathrm{TM}}$, 4 EDDY ${ }^{\circledR}, 5$ KONTROLLGRUPPE) …..................................... 38

ABBILDUNG 19: VERTEILUNG DER SCORES FÜR DEBRIS IM KORONALEN WURZELKANALANTEIL (BOX-

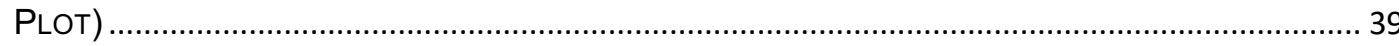

ABBILDUNG 20: VERTEILUNG DER SCORES FÜR DEBRIS IM KORONALEN WURZELKANALANTEIL

(DIAGRAMM).

ABBILDUNG 21: VERTEILUNG DER SCORES FÜR DEBRIS FÜR DEN APIKALEN WURZELKANALANTEIL

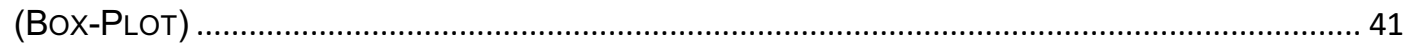

ABBILDUNG 22: VERTEILUNG DER SCORES FÜR DEBRIS IM APIKALEN WURZELKANALANTEIL

(DIAGRAMM).

ABbildung 23: VeRTEILUNG der SCORES FÜR SMEAR LAYER NACH GRUPPEN. (1 HANDSPÜLUNG, 2

ENDOACTIVATOR®, 3 IRRISAFE ${ }^{\text {TM }}$, 4 EDDY ${ }^{\circledR}, 5$ KONTROLLGRUPPE)......

ABBILDUNG 24: VERTEILUNG DER SCORES FÜR SMEAR LAYER FÜR DEN KORONALEN

WURZELKANALANTEIL (BOX-PLOT).

ABBILDUNG 25: VERTEILUNG DER SCORES FÜR SMEAR LAYER IN DEN KORONALEN

WURZELKANALANTEILEN (DIAGRAMM).

ABBILDUNG 26: VERTEILUNG DER SCORES FÜR SMEAR LAYER IM APIKALEN WURZELKANALANTEIL.

(BOX-PLOT) 
ABBILDUNG 27: VERTEILUNG DER SCORES FÜR SMEAR LAYER IM APIKALEN WURZELKANALANTEIL

(DIAGRAMM). 48

ABbildung 28: VeRTEILUNG der SCORES FÜR DEBRIS IN GRUPPE 1 (DyNAMISCHE HANDSPÜLUNG) 49 ABBILDUNG 29: VERTEILUNG DER SCORES FÜR SMEAR LAYER IN GRUPPE 1 (DYNAMISCHE

HANDSPÜLUNG). 50

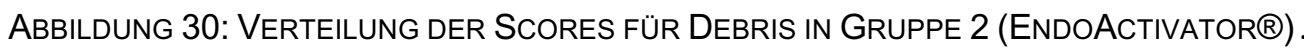
51

ABBILDUNG 31: VERTEILUNG DER SCORES FÜR SMEAR LAYER IN GRUPPE 2 (ENDOACTIVATORß)......52

AbBILdUNG 32: VeRTEILUNG deR SCORES FÜR Debris IN GRUPPE 3 (IRRISAFE ${ }^{\text {TM }}$ ).......................... 53

ABBILDUNG 33: VERTEILUNG DER SCORES FÜR SMEAR LAYER IN GRUPPE 3 (IRRISAFE TM).................. 54

ABBILDUNG 34: VERTEILUNG DER SCORES FÜR DEBRIS IN GRUPPE 4 (EDDY $\left.{ }^{\circledR}\right)$.................................. 55

ABBILDUNG 35: VERTEILUNG DER SCORES FÜR SMEAR LAYER IN GRUPPE 4 (EDDY $\left.{ }^{\circledR}\right)$.......................... 56

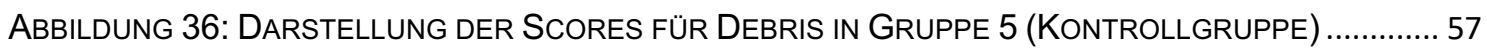

ABBILDUNG 37: DARSTELLUNG DER SCORES FÜR SMEAR LAYER IN GRUPPE 5 (KONTROLLGRUPPE) ... 58

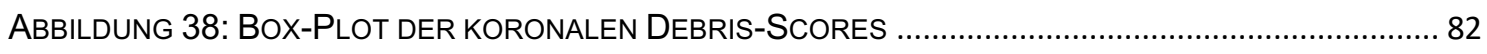

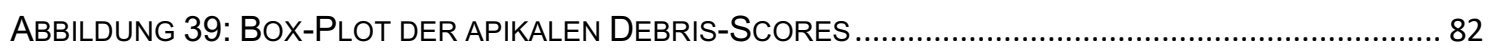

ABBILDUNG 40: BOX-PLOT DER KORONALEN SMEAR LAYER-SCORES ……………………………....... 83

ABBILDUNG 41: BOX-PLOT DER APIKALEN SMEAR LAYER-SCORES........................................................ 83 
Tabellenverzeichnis

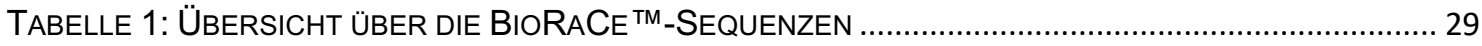

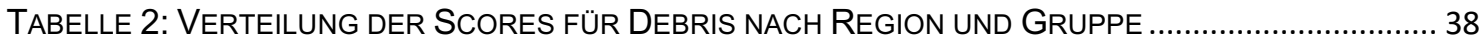

TABELLE 3: VERTEILUNG DER SCORES FÜR SMEAR LAYER NACH REGION UND GRUPPE ...................... 43

TABELLE 4: ERgEBNISSE dES KRUSKAL-WALLIS-TESTS FÜR DEBRIS KORONAL (STATISTICA) .............. 59

TABELLE 5: ERgEBNISSE DES KRUSKAL-WALliS-TESTS FÜR DEBRIS APIKAL (STATISTICA) ..................60

TABELLE 6: Zusammenfassung DER ERGEBNISSE DER MANN-WHITNEY-U-TESTS FÜR DEBRIS APIKAL

TABELLE 7: ZUSAMMENFASSUNG DER NACH BONFERRONI KORRIGIERTEN P-WERTE FÜR DEBRIS APIKAL

TABELLE 8: ERGEBNISSE DES KRUSKAL-WALLIS-TESTS FÜR SMEAR LAYER KORONAL (STATISTICA) ... 62

TABELLE 9: ERGEBNISSE DES KRUSKAL-WALLIS-TESTS FÜR SMEAR LAYER APIKAL (STATISTICA)......... 63

TABELLE 10: ZUSAMMENFASSUNG DER ERGEBNISSE DER MANN-WHITNEY-U-TESTS FÜR SMEAR LAYER APIKAL 64

TABELLE 11: NACH BONFERRONI KORRIGIERTE P-WERTE FÜR DIE VARIABLE SMEAR LAYER APIKAL .....64

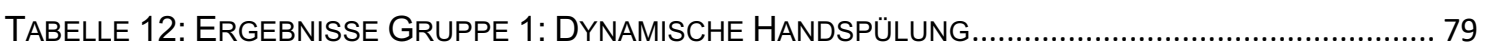

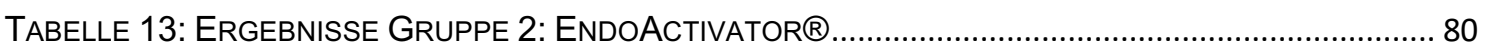

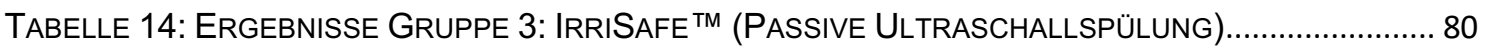

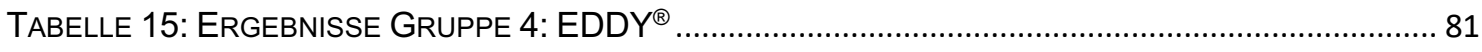

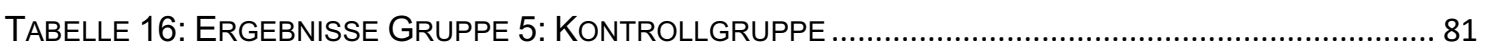

TABELLE 17: ERgEBNISSE DES MANN-WHITNEY-U-TESTS FÜR DEBRIS APIKAL (GRUPPE 1 GEgEN

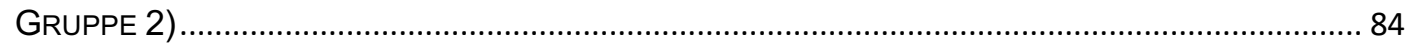

TABelle 18: ERgebnisse des MANN-Whitney-U-Tests FÜr DEBRIS APIKAL (GRUPPE 1 Gegen

GRUPPE 3). 84

TABELLE 19: ERgEBNISSE DES MANN-WhItNEY-U-TESTS FÜR DEBRIS APIKAL (GRUPPE 1 GEgEN GRUPPE 4)

TABelle 20: ERgebnisse des MANN-Whitney-U-Tests für Debris APIKAL (GRUPPe 1 Gegen GRUPPE 5).

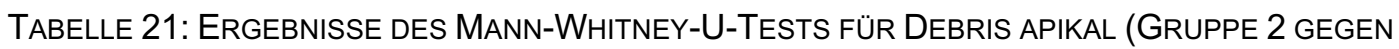

GRUPPE 3).

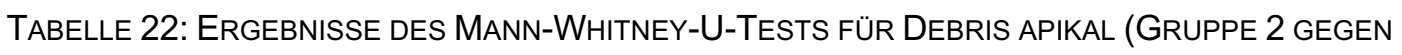
GRUPPE 4).

TABelle 23: ERgebnisse des MANN-Whitney-U-Tests Für DEBRIS APIKAL (GRUPPE 2 GEgEN

GRUPPE 5)

TABelle 24: ERgebNisSe des MAnN-Whitney-U-Tests für Debris APIKAL (GRUPPe 3 gegen GRUPPE 4) 85

TABelle 25: ERgebNisse des MANN-Whitney-U-Tests für Debris APIKAL (GRUPPE 3 gegen GRUPPE 5) 
TABelle 26: ERgeBnisse des MANN-Whitney-U-Tests für DEBRIS APIKAL (GRUPPE 4 GEgEN GRUPPE 5)

TABELLE 27: ERGEBNISSE DES MANN-WHITNEY-U-TESTS FÜR SMEAR LAYER APIKAL (GRUPPE 1 GEGEN GRUPPE 2)..... 86

TABelle 28: ERgebnisse des ManN-Whitney-U-Tests FÜr SMEAR LAYER APIKAL (GRUPPE 1 GEGEN GRUPPE 3)

TABELle 29: ERgeBnisse DES MANN-WhitNEY-U-TestS FÜr SMEAR LAYER APIKAL (GRUPPE 1 GEGEN GRUPPE 4).

TABELle 30: ERgeBnisse des MANN-Whitney-U-TESTS FÜR SMEAR LAYER APIKAL (GRUPPE 1 GEGEN GRUPPE 5).....

TABELle 31: ERgeBNisSe des MANN-Whitney-U-TESTS FÜR SMEAR LAYER APIKAL (GRUPPE 2 GEGEN GRUPPE 3).

TABELle 32: ERgeBNisSE DES MANN-Whitney-U-TESTS FÜR SMEAR LAYER APIKAL (GRUPPE 2 GEGEN GRUPPE 4).

TABELLE 33: ERGEBNISSE DES MANN-WHITNEY-U-TESTS FÜR SMEAR LAYER APIKAL (GRUPPE 2 GEGEN GRUPPE 4)...

TABelle 34: ERgebnisse des ManN-Whitney-U-Tests Für SMEAR LAYER APIKAL (GRUPPE 3 GEGEN GRUPPE 4)..... 88

TABELle 35: ERgeBNisSe des MANN-Whitney-U-TESTS FÜR SMEAR LAYER APIKAL (GRUPPE 3 GEGEN GRUPPE 5).

TABELle 36: ERgeBnisse des MANN-Whitney-U-TESTS FÜR SMEAR LAYER APIKAL (GRUPPE 4 GEGEN GRUPPE 5).

TABELLE 37: HERSTELLERVERZEICHNIS UND MATERIALLISTE 
Abkürzungsverzeichnis

$\begin{array}{ll}\text { CHX } & \text { Chlorhexidin } \\ \text { CPM } & \text { Counts per Minute } \\ \text { DGZMK } & \begin{array}{l}\text { Deutsche Gesellschaft für Zahn-, Mund- und } \\ \text { Kieferheilkunde }\end{array} \\ \text { E. faecalis } & \text { Enterococcus faecalis } \\ \text { EDTA } & \text { Ethylendiamintetraacetat } \\ \text { EDTANa4 } & \text { Tetranatriumethylendiamintetraacetat } \\ \text { NaOCI } & \text { Natriumhypochlorit } \\ \text { NiTi } & \text { Nickel-Titan } \\ \text { PUI } & \text { Passive Ultrasonic Irrigation }\end{array}$




\section{$1 \quad$ Einleitung}

Der Erhalt des Zahnes sollte für jeden Zahnarzt stets das höchste Ziel seiner Tätigkeit darstellen. Bei größeren Defekten wie einer caries profunda, die definitionsgemäß bis in Pulpanähe reicht, kann es zu einer initial reversiblen oder im späteren Verlauf irreversiblen Schädigung der zunächst sterilen Pulpa kommen. Ist die Pulpa durch Bakterieninvasion irreversibel entzündet, stellt die Wurzelkanalbehandlung den Versuch dar, den infizierten Zahn zu retten. Ziel der Wurzelkanalbehandlung ist zunächst die Schmerzfreiheit des Patienten. Dabei ist allerdings zu beachten, dass Pulpitiden auch zunächst völlig asymptomatisch verlaufen können. Mit Hilfe steriler Instrumente wird das erkrankte pulpale Gewebe mechanisch aus dem Zahn entfernt. Es schließt sich eine chemische Reinigung an, bei der das Wurzelkanalsystem mit antibakteriellen Lösungen desinfiziert wird, wodurch die bakterielle Besiedlung minimiert werden soll. Bei der Spülung des Wurzelkanalsystems lässt es sich aufgrund seiner dreidimensionalen Komplexität nicht vermeiden, dass Bakterien und nekrotisches Gewebe, der sogenannte Debris, im System verbleiben. Zusätzlich hinterlässt die mechanische Bearbeitung eine Schmierschicht auf der Wurzelkanalwand, den smear layer, der zum einen bakteriell kontaminiert ist und zum anderen die Dichtigkeit der späteren Wurzekanalfüllung kompromittiert. Sowohl Debris als auch Schmierschicht sollen durch eine effiziente Desinfektion möglichst vollständig entfernt werden. Um die Wirkung der Spüllösungen zu maximieren, wurden in den letzten Jahrzehnten verschiedene Möglichkeiten der Aktivierung der Lösungen im Wurzelkanalsystem entwickelt. Eine kleine Auswahl solcher Systeme ist Bestandteil der vorliegenden Studie. Nach der mechanischen sowie chemischen Reinigung des Wurzelkanalsystems erfolgt dessen dichte Füllung. Als Abschluss der endodontischen Behandlung steht die postendodontische Versorgung des Zahnes im koronalen Bereich, welche den Erfolg der endodontischen Behandlung langfristig sicherstellen soll. Als entscheidend für den Erfolg der Behandlung wird die strikte Einhaltung einer 
Kette von aseptischen bzw. antiseptischen Arbeitsschritten angesehen, in deren Mittelpunkt die chemische Desinfektion steht. 


\section{Literaturübersicht}

\subsection{Die Wurzelkanalbehandlung}

Die Endodontologie stellt einen nicht unerheblichen Teilbereich der konservierenden Zahnheilkunde dar. Die Europäische Gesellschaft für Endodontologie (European Society of Endodontology, ESE) definiert die Endodontie als Lehre von der Form, Funktion und Gesundheit der Pulpa und der periradikulären Gewebe. In das Feld der Endodontie gehören ebenso die Verletzungen und Erkrankungen der Pulpa und der periradikulären Gewebe sowie deren Prävention und Therapie (Europäische Gesellschaft für Endodontologie: Qualitätsrichtlinien Endodontischer Behandlungen 2006). Die primären Ziele einer endodontischen Behandlung sind dabei der Zahnerhalt und die Schmerzfreiheit des Patienten. Die Hauptursache für eine Schädigung der Pulpa liegt zumeist in einer kariösen Läsion, aber auch Traumata, Parodontopathien oder restaurative Maßnahmen können zur Pulpaschädigung führen. Torabinejad et al. (2002) erläuterten, dass die Zielsetzung der endodontischen Behandlung in der Entfernung von erkranktem Gewebe, der Eliminierung von Bakterien im Wurzelkanal sowie in den Dentintubuli und der Prävention einer Rekontamination nach der Behandlung bestehe. Diese Zielsetzung ließe sich durch Präparation, Säuberung und Desinfektion des Kanalsystems erreichen. Abschließend sei es nötig, die Wurzelkanäle mit einer dreidimensionalen Füllung zu versehen und für einen dichten koronalen Verschluss zu sorgen (Torabinejad et al. 2002). Durch diese Arbeitsschritte soll eine weitestgehende Bakterienreduktion erreicht werden, so dass vorhandene Entzündungen abklingen und keine neuen entstehen können. Die Präparation kann dabei mit Handinstrumenten oder maschinellen Geräten durchgeführt werden. Nach Hülsmann et al. (2005) ist bei der Präparation auf folgende Punkte zu achten:

- Entfernung vitalen und nekrotischen Gewebes aus den Hauptkanälen

- Schaffung von genügend Platz zur Spülung und Medikamentenapplikation 
- Erhalt der ursprünglichen Anatomie des Wurzelkanals, vor allem im apikalen Bereich

- Vermeidung iatrogener Schädigungen am Wurzelkanalsystem und der Wurzelstruktur

- Das Füllen des Wurzelkanals soll durch die Präparation erleichtert werden

- Vermeidung weiterer Irritation oder Schädigung der periradikulären Gewebe

- Erhalt von gesundem Wurzeldentin.

Während der Präparation der Wurzelkanäle kommt es zur Entstehung des sog. smear layer (Schmierschicht). Eine Übersicht über dessen Beschaffenheit und seinen Einfluss auf die Wurzelkanalbehandlung findet sich im Kapitel 2.2. Bei der rein mechanischen Präparation der Wurzelkanäle bleiben aufgrund der Komplexität des endodontischen Systems bis zu $35 \%$ der Kanaloberflächen unbearbeitet (Peters et al. 2001). In ovalen Wurzeln kann dieser Wert noch deutlich höher liegen. So können bei entsprechend komplexer Wurzelkanalanatomie bis zu $79 \%$ der Wurzelkanalwände uninstrumentiert bleiben (Paqué et al. 2010). Auch Ørstavik et al. (1991) zeigten, dass eine rein mechanische Präparation nicht in der Lage ist, eine ausreichende Bakterienfreiheit zu gewährleisten. Um auch in kleinen Nebenkanälen eine ausreichende Bakterienreduktion zu erreichen und gleichzeitig die Schmierschicht zu entfernen, ist es daher unerlässlich, neben der mechanischen Präparation eine chemische Desinfektion des Systems vorzunehmen. Die Spülung selbst wird auch als „Chemisches Debridement“ bezeichnet, in Verbindung mit der vorangehenden mechanischen Komponente spricht man auch von der „chemo-mechanischen“ Präparation des Kanalsystems (Hülsmann 2008). Dabei stehen dem Zahnarzt heute eine Vielzahl unterschiedlicher Lösungen und Techniken zur Verfügung, auf die in den Kapiteln 2.3 und 2.4 näher eingegangen wird. Ein eindeutiger Konsens über die geeignetste Spülung des Wurzelkanals besteht dabei nicht. Der Verschluss des Wurzelkanals erfolgt in der Regel mit Guttapercha in Verbindung mit einem Sealer. Die Guttapercha bildet dabei ein volumenstabiles Kernmaterial, der erhärtende Sealer dient dazu, Inkongruenzen zwischen der Kanalwand und der Guttapercha auszugleichen (Rödig et al. 2009). Ziel ist ein bakteriendichter Verschluss des 
Wurzelkanalsystems, um eine Rekontamination mit Bakterien zu vermeiden. Häufig wird vor der definitiven Füllung der Wurzelkanäle eine medikamentöse Einlage auf Kalziumhydroxid- oder Chlorhexidinbasis in das Wurzelkanalsystem eingebracht. Diese soll bewirken, dass die Bakterienreduktion verbessert wird. Die optimale Liegedauer einer solchen Einlage beträgt zwischen 8 und 14 Tagen, eine längere Dauer zeigt keine nennenswerte weitere Keimreduktion (Hülsmann 2008). Während dieser Zeit wird der Zahn koronal temporär verschlossen. Nach Entfernung des Medikamentes und erneuter Spülung des Wurzelkanalsystems können die definitive Füllung und Restauration des Zahnes erfolgen.

\subsection{Smear Layer}

Der smear layer - zu deutsch „Schmierschicht“ - entsteht bei jeder Präparation an der Zahnhartsubstanz und legt sich unregelmäßig als inhomogene Schicht auf die bearbeiteten Oberflächen. In der Endodontie spielt der smear layer eine für den Erfolg der Behandlung wichtige Rolle. Nach der Wurzelkanalpräparation bedeckt dieser die Wurzelkanaloberflächen. Dabei werden die offenliegenden Dentintubuli infiltriert und somit zu einem großen Teil verschlossen. Dies hat zur Folge, dass die Desinfektion des Wurzelkanalsystems deutlich erschwert wird, da die Lösungen nicht ausreichend in die Tiefe des Dentins einwirken können, um dort befindliche Mikroorganismen abzutöten. Des Weiteren wird die spätere Dichtigkeit der Wurzelkanalfüllung negativ beeinflusst, da der Sealer bzw. die Guttaperchafüllung nicht den optimalen Verbund zur Wurzelkanalwand herstellen können.

Bestandteile des smear layer sind unter anderem Dentin, nekrotisches und vitales Gewebe, Reste von Odontoblastenfortsätzen, Pulpagewebe und Bakterien (McComb und Smith 1975). Zusätzlich kann die Schmierschicht Dentinliquor und Spülflüssigkeit enthalten und besitzt somit organische und anorganische Anteile. Mit der genaueren morphologischen Struktur der Schmierschicht beschäftigten sich Eick et al. (1970). Sie fanden, dass die Schicht vorwiegend Zahnhartsubstanzpartikel in einer Größe zwischen 0,5 und $15 \mu \mathrm{m}$ enthält, die in eine organische Matrix mit einer Stärke von ca. 0,5 
$\mu \mathrm{m}$ eingebettet sind. Brannström et al. (1980) sprechen von einem amorphen Erscheinungsbild des smear layer.

Der smear layer legt sich als inhomogene Schicht auf die präparierte Wurzelkanalwand. Dabei werden die offenliegenden Dentintubuli abgedeckt. Mader et al. (1984) zeigten, dass die Schmierschicht nicht nur oberflächlich auf den Dentintubuli aufliegt, sondern sie in eine Tiefe von bis zu $40 \mu \mathrm{m}$ penetriert. Dieses Phänomen wird auch als „smear plugs“ (Pashley 1992) bezeichnet. Es führt zu einer schlechteren Penetration von medikamentösen Einlagen in die Dentintubuli und beeinflusst die spätere Adaptation des Füllungsmaterials an die Wurzelkanalwand (Violich und Chandler 2010). Weiterhin sind antibakterielle Spülungen in den aufgrund der Blockade durch die smear plugs schlecht oder nicht zugänglichen Bereichen des Wurzelkanalsystems weniger wirksam (Baumgartner und Mader 1987; Yamada et al. 1983). Ørstavik und Haapasalo (1990) sprechen in diesem Zusammenhang von einer Verzögerung der Wirksamkeit der Spülungen, nicht aber von einer Aufhebung deren Wirkung.

Ein weiterer entscheidender Punkt in Bezug auf den smear layer ist die Frage, inwieweit er Bakterien beherbergt, die eine erfolgreiche endodontische Behandlung gefährden können. Dazu stellten Baumgartner und Mader (1987) fest, dass die Schmierschicht neben eigenen Mikroorganismen auch andere Mikroorganismen, die bereits im Wurzelkanal vorhanden sind, bedeckt und sie somit einschließt. Diese Bakterien können auch nach erfolgter chemischer Desinfektion zurückbleiben (Shovelton 1964) und sich sogar wieder vermehren (Meryon und Brook 1990). Sjögren et al. (1997) sehen das Verbleiben von Bakterien der Gattung Actinomyces im Wurzelkanal als Ursache für ein Scheitern der endodontischen Behandlung und empfehlen die Einlage eines antibakteriellen Medikaments vor der Füllung des Wurzelkanals. Heute ist die Frage, ob der smear layer bei der Wurzelkanalbehandlung entfernt werden oder aber belassen werden sollte, nicht abschließend beantwortet. Timpawat et al. (2001) beschrieben, dass durch ein Belassen des smear layer die Gefahr eines microleakage (Mikroundichtigkeit) reduziert werden kann. Dies ist eine Bestätigung der Ergebnisse von Groves Cooke et al. (1976), die ebenfalls eine Zunahme von 
microleakages an Zähnen beobachteten, an denen die Schmierschicht entfernt wurde. Chailertvanitkul et al. (1996) fanden keinen signifikanten Unterschied bei Belassen der Schmierschicht in Bezug auf die Entstehung eines microleakage.

Andere Studien untersuchten, welche Auswirkung der smear layer auf die Permeabilität des Dentins hat. Ein Belassen des smear layer kann dazu beitragen, die Permeabilität des Dentins herabzusetzen und somit eine Penetration von Mikroorganismen zu erschweren (Pashley et al. 1981). Im Gegensatz dazu steht die Studie von Meryon und Brook (1990), die die Penetration von drei oralen Bakteriengattungen in das Wurzelkanaldentin untersuchten. Das Vorhandensein einer Schmierschicht hatte keinen Einfluss auf die Permeabilität (Meryon und Brook 1990).

Thema weiterer Studien war die Frage, inwieweit der smear layer die Adaptation des Wurzelfüllungsmaterials an die Kanalwand beeinflussen kann. Gençoğlu et al. (1993) untersuchten dies in zwei Studien. Dabei stellten sie fest, dass die Adaptation der Guttapercha nach der Entfernung des smear layer deutlich verbessert war (Gençoğlu et al. 1993a). Diese Ergebnisse bestätigten sich in der zweiten Studie. Auch hier verhalf die Entfernung des smear layer in allen Gruppen zu einer dichteren Füllung des Wurzelkanals (Gençoğlu et al. 1993b). In einer Metaanalyse untersuchten Shahravan et al. (2007) 26 Studien aus den Jahren 1975 bis 2002, die sich mit der Frage der Auswirkung des smear layer auf die Dichtigkeit der Füllung beschäftigten. In $53,8 \%$ der Fälle fanden sie dabei keinen signifikanten Unterschied, 41,5\% der Studien favorisierten eine Entfernung der Schmierschicht und lediglich 4,7\% sprachen sich für einen Erhalt der Schicht aus. Aufgrund dieser Ergebnisse schlussfolgerten sie, dass die Entfernung der Schmierschicht einen flüssigkeitsdichten Verschluss des Wurzelkanalsystems verbessert. Auch Violich und Chandler (2010) sprachen sich für eine Entfernung der Schmierschicht aus, da dies die Desinfektion des Wurzelkanalsystems verbessere und eine dichtere Füllung ermögliche. Die Methode der Wahl sei dabei der Gebrauch von NaOCI und EDTA. Auch die Deutsche Gesellschaft für Zahn-, Mund- und Kieferheilkunde (DGZMK) und die Deutsche Gesellschaft für Zahnerhaltung (DGZ) empfehlen in ihrer gemeinsamen 
wissenschaftlichen Leitlinie zur Wurzelkanalspülung die Entfernung des smear layer (Leitlinie Wurzelkanalspülung 2006).

\subsection{Wurzelkanaldesinfektion: Techniken}

\subsection{1 Übersicht über aktuelle Spültechniken}

Heute stehen dem Zahnarzt eine Vielzahl unterschiedlicher Spültechniken zur Verfügung, auf die im Folgenden kurz eingegangen wird. Die in dieser Studie verwendeten Spültechniken werden unter 2.3.2 näher erläutert. Ausgehend von der Handspülung wurden im Laufe der Zeit neue Möglichkeiten gesucht, die Desinfektion im Kanalsystem zu verbessern. Vor allem in nicht mechanisch bearbeiteten Anteilen des endodontischen Systems, also in kleinen Seitenkanälen, Dentintubuli und im Wurzelspitzenbereich, versprachen neue Möglichkeiten der Spülung eine bessere Keimreduktion. Dabei lag das Augenmerk weniger auf der Suche nach neuen Spüllösungen, sondern es stand die Frage im Fokus, wie geeignete Flüssigkeiten besser in größere Teile des verzweigten Systems vordringen können, um eine bessere Reduktion von Mikroorganismen erzielen zu können. Neben der Möglichkeit, mit Bürsten, z. B. der Canalbrush (Coltène/Whaledent, Langenau), eine zusätzliche mechanische Komponente einzusetzen, werden Spülkanülen angeboten, die mit einer Bürste kombiniert sind, z. B. NaviTip Fx (Ultradent, Köln). Es wurden verschiedene Möglichkeiten der Aktivierung von Spülflüssigkeiten entwickelt. Bevorzugt werden heute Systeme eingesetzt, die per Schall oder Ultraschall aktiviert werden. $\mathrm{Zu}$ den schallaktivierten Systemen gehört z. B. der EndoActivator® (Dentsply Sirona, York, Pennsylvania, USA). Schallaktivierte Systeme arbeiten im Bereich von ca. 1$6 \mathrm{KHz}$. Einen Vertreter der ultraschallaktivierten Systeme stellt zum Beispiel IrriSafe $^{\mathrm{TM}}$ dar (Acteon Group, Norwich, England). Bei der Aktivierung der Spülflüssigkeiten per Schall oder Ultraschall kommt es zu einer effektiveren Reinigung des Kanalsystems, bedingt durch spezielle hydrodynamische Effekte, die in der Spüllösung entstehen. Diese werden in 2.3.2.2 und 2.3.2.3 näher erläutert. Die Ultraschallaktivierung sollte stets passiv erfolgen, um einen unkontrollierten Dentinabtrag zu vermeiden. Weitere Ansätze für eine Desinfektion des Kanalsystems stellen die Photodynamische Therapie (PDT) 
und der Einsatz von Lasern dar. Zu allen genannten Techniken stellt die Literatur bereits viele Studien zur Verfügung, die jedoch zu unterschiedlichen Ergebnissen kommen. Daher herrscht kein eindeutiger Konsens über die geeignetste Technik für eine effektive Desinfektion des Endodonts.

\subsubsection{In dieser Studie verwendete Spültechniken}

\subsubsection{Dynamische Handspülung}

Die Desinfektion des Kanalsystems per Handspülung stellt an das benötigte Material die geringsten Anforderungen. In der Regel werden konventionelle Kunststoffspritzen mit einem Volumen von $5 \mathrm{ml}$ verwendet. Als Spülkanülen kommen stumpfe Kanülen mit geringem Durchmesser von normalerweise ISO 40 oder kleiner zum Einsatz. Die Kanülen werden entsprechend vorgebogen, um ein einfaches Einführen in den Wurzelkanal zu gewährleisten. Entscheidend für die Effektivität der Handspülung ist die Eindringtiefe der Kanülenspitze. Die Reinigungswirkung der Spülung endet wenige Millimeter nach Austritt aus der Kanüle (Schäfer 2007). Um auch im apikalen Bereich eine ausreichende Reinigungsleistung zu erzielen, muss die Kanüle demzufolge bis auf 2-3 mm vor Arbeitslänge eingeführt werden können. Die Spülnadel sollte bis zu einem spürbaren Widerstand in den Kanal eingebracht und dann 1-2 mm zurückgezogen werden (Schäfer 2007). Auf diese Weise lässt sich ein Überpressen der Spülflüssigkeit in periapikales Gewebe vermeiden und zusätzlich kann die Spülflüssigkeit ungehindert nach koronal abfließen (Chow 1983; Schäfer 2007; Walters et al. 2002). Dies setzt eine ausreichend weite Präparation des Wurzelkanals mindestens bis zur ISO-Größe 35 voraus. Diese Präparationsgröße empfiehlt Schäfer (2007), ebenso wie einen Kanülendurchmesser von 0,3 mm (ISO 30). Der Nachteil kleinerer Kanülen liegt im erhöhten Kraftaufwand während der Spülung und in einer erhöhten Verstopfungsgefahr der Kanüle. Neben den konventionellen Spülkanülen gibt es spezielle Kanülen, die über seitliche Öffnungen in der Kanülenwand verfügen (Kahn et al. 1995). Diese Kanülen müssen weiter in den Kanal eingebracht werden als konventionelle Kanülen, um im apikalen Bereich eine effektive Reinigungswirkung zu erzielen (Šnjarić et al. 2012). Bei der Handspülung muss stets passiv, d. h. langsam und ohne Druck gearbeitet 
werden. Die Spülflüssigkeit muss ungehindert nach koronal abfließen können und darf nicht versehentlich über das Foramen apicale hinaus appliziert werden, um Schädigungen der periapikalen Gewebe zu vermeiden. Die koronal abfließende Lösung sollte direkt abgesaugt werden, um einen Übertritt in die Mundhöhle auszuschließen. Wie bei anderen Spülmethoden auch, sollten während der Applikation leicht pumpende Vertikalbewegungen ausgeführt werden (dynamische Handspülung) (Park et al. 2013). Für eine maximale Effizienz wird eine Flussrate von $4 \mathrm{ml} / \mathrm{min}$ empfohlen (Park et al. 2013). Wie bei allen Spülmethoden ist auf eine ausreichende Einwirkzeit der Spülflüssigkeiten zu achten. Die Handspülung kann mit allen gängigen Spüllösungen durchgeführt werden. Initial nach der Kavitätenpräparation sollte die Pulpakammer mit Natriumhypochlorit gefüllt werden, da dies einerseits die Einwirkzeit der Spüllösung verlängert und andererseits als Gleitmittel für die Präparationsinstrumente dient (Schäfer 2007). Es sollen flexible Spülkanülen mit Sicherheitsspitze verwendet und diese der Kanalkrümmung entsprechend vorgebogen werden, um eine ausreichende apikale Reinigung zu gewährleisten (Schäfer 2007).

\subsubsection{EndoActivator}

Der EndoActivator® der Firma Dentsply ist ein batteriebetriebenes Gerät zur schallgestützten Aktivierung von Spülflüssigkeiten. Das System wurde von Dr. Cliff Ruddle, Dr. Robert H. Sharp und Dr. Pierre Machtou entwickelt und besteht aus einem kabellosen Handstück, das mit zwei AA-Batterien betrieben wird, und dazu passenden Spülspitzen aus Polymer. Die aus Medical-grade-Polymer gefertigten Spitzen sind in den Größen klein (15/.02), mittel (25/.04) und groß (35/.04) erhältlich. Die Länge der Spitzen beträgt einheitlich $22 \mathrm{~mm}$ und sie tragen Längenmarkierungen bei 18, 19 und $20 \mathrm{~mm}$. Das Handstück erlaubt einen Betrieb mit einer Frequenz von 2000, 6000 oder 10.000 cpm (Counts per Minute). Die für die Aktivierung der Spülflüssigkeit empfohlene Frequenz beträgt 10.000 cpm. Die Schallaktivierung durch den EndoActivator® erzeugt eine Flüssigkeitshydrodynamik, welche die Desinfektion des Kanalsystems verbessern soll. Bei der Anwendung wird die entsprechende Spülspitze auf das Handstück aufgesteckt. Nachdem das Kanalsystem mit Spülflüssigkeit gefüllt wurde, wird die Spülspitze bis auf 2 
$\mathrm{mm}$ vor Arbeitslänge eingebracht. Die Spitze muss dabei ausreichend Platz für Schwingung haben. Während der Aktivierung über 30-60 Sekunden werden vertikale Pumpbewegungen mit einer Hubhöhe von 2-3 mm ausgeführt. Ruddle (2002) bestätigt, dass durch die Zusatzbewegungen ein die Reinigung verbesserndes, hydrodynamisches Phänomen entsteht. Die Dauer der Aktivierung muss mindestens 30 Sekunden betragen bei einer Gesamteinwirkzeit der Spüllösung von 60 Sekunden, wobei es keine Rolle spielt, ob die Aktivierung zu Beginn der Einwirkzeit oder gegen Ende ausgeführt wird (Niu et al. 2014).

Die Spülung mit dem EndoActivator® zeigt eine deutlich bessere Entfernung des Smear-Layers besonders in der mittleren Kanalregion im Vergleich zur Spülung per Hand oder mit der Canal-Brush (Singh et al. 2014). Andere Studien bescheinigen der schallaktivierten Spülung mit dem EndoActivator® eine der passiven Ultraschallspülung überlegene Reinigungswirkung. So beschreiben Khalap et al. (2016) vor allem in Verbindung mit dem ProTaper Next-System (Dentsply, York, Pennsylvania, USA) zur Präparation eine bessere Reinigung als durch die ultraschallaktivierte Spülung. Auch mit auf $60^{\circ} \mathrm{C}$ erwärmtem Natriumhypochlorit erweist sich die Reinigungsleistung durch den EndoActivator $\circledast$ gegenüber der Ultraschallspülung als effektiver (Guo et al. 2014). Die Wurzelkanaldesinfektion mit dem EndoActivator® erlaubt über die gesamte Kanallänge eine bessere Entfernung des smear layer im Vergleich zur passiven Ultraschallspülung, jedoch zeigt sie sich der Kanalreinigung durch das EndoVac System (Discus Dental, Culver City, USA) unterlegen (Mancini et al. 2013). Eine auf die Entfernung des smear layer im koronalen Bereich beschränkte bessere Wirksamkeit gegenüber der passiven Ultraschallspülung wurde von Rödig et al. (2010) festgestellt. Zu diesem Ergebnis kamen auch Caron et al. (2010). Keine Unterschiede in der Reinigungsleistung des EndoActivators ${ }^{\circledR}$ gegenüber Handspülung und ultraschallaktivierter Spülung hingegen beschrieben Klyn et al. (2010) sowie Uroz-Torres et al. (2010). In Hinblick auf die Entfernung des endodontischen Problemkeims Enterococcus faecalis war der EndoActivator ${ }^{\circledR}$ der passiven Ultraschallspülung unterlegen (Rico-Romano et al. 2016). Die Gefahr der apikalen Extrusion von Spülflüssigkeit ist bei der Benutzung des EndoActivators $\AA$ als gering einzustufen, unabhängig von der 
Präparationsgröße des Kanals (Yost et al. 2015). Die Verwendung des EndoActivators $₫$ kann effektiv dazu beitragen, die postoperativen Schmerzen nach der Wurzelkanalbehandlung im Gegensatz zur Spülung per Hand zu reduzieren und somit die Notwendigkeit einer unterstützenden Schmerzmedikation zu minimieren (Ramamoorthi et al. 2015).

\subsubsection{IrriSafe ${ }^{\mathrm{TM}}$}

Die IrriSafe ${ }^{\mathrm{TM}}$ Spülspitzen wurden von der Firma Satelec in Zusammenarbeit mit der holländischen ACTA Universität in Amsterdam entwickelt. IrriSafe ${ }^{\mathrm{TM}}$ Spülspitzen können bei Spülprotokollen mit passiver Ultraschallspülung (PUI: Passive Ultrasonic Irrigation) und Natriumhypochlorit als Spülflüssigkeit eingesetzt werden. Der Betrieb erfolgt dabei mit einem kompatiblen, piezoelektrischen Ultraschallgenerator wie z. B. dem VDW Ultra, der auch in dieser Studie Anwendung fand. Die Spülspitze wird dabei in Schwingungen im Ultraschallbereich $(20 \mathrm{KHz}-40 \mathrm{KHz})$ versetzt. Die Spitzen des IrriSafe ${ }^{\mathrm{TM}}$ Systems bestehen aus medizinischem Stahl und sind in den Größen 20/21, 20/25, 25/21 und 25/25 erhältlich. Dabei gibt die erste Zahl jeweils den Durchmesser an (20 entspricht hierbei einem Durchmesser von 0,2 mm), die zweite Zahl bezeichnet die Länge der Spitze in Millimetern. Neben den geringen Durchmessern der Spitzen weisen diese parallel verlaufende, nicht schneidende Seiten sowie eine stumpfe Spitze auf. IrriSafe ${ }^{\mathrm{TM}}$ sollte bei der Aktivierung einen Abstand von $1 \mathrm{~mm}$ zur Wurzelkanalwand aufweisen und ungehindert schwingen können. Empfohlen wird dabei ein kontinuierlicher Austausch der Spülflüssigkeit über drei Minuten oder ein Austausch in drei Intervallen und Spülung für je eine Minute.

Die Effektivität der passiven Ultraschallspülung mit IrriSafe ${ }^{\mathrm{TM}}$ soll durch Effekte wie Mikroströmungen, Mikrokavitationseffekte und den Thermoeffekt verbessert werden. Durch die Schwingung im Ultraschallbereich kommt es innerhalb der Spülflüssigkeit zum sogenannten acoustic streaming (Ahmad et al. 1987). Dabei wird die die Feile umgebende Flüssigkeit in eine derart starke Schwingung versetzt, dass die erzeugte Energie die Kohäsionskräfte der Flüssigkeitsmoleküle übersteigt. Dabei entstehen Luftblasen, die sogleich wieder kollabieren. Durch dieses Phänomen kommt es zur Energieübertragung auf die Wurzelkanalwand, ohne dass die Feile selbst die 
Wand berührt. Die erzeugte Energiemenge ist dabei größer als bei der Verwendung von aktiven Ultraschallfeilen (Plotino et al. 2007). Wenn die Feile frei schwingen kann, ist das Phänomen des acoustic streaming besser wirksam als bei Kontakt mit der Wurzelkanalwand (Roy et al. 1994).

Viele Studien bestätigen der Reinigung mit Hilfe der PUI eine verbesserte Entfernung des smear layer. Unter anderem zeigten Ordinola-Zapata et al. (2014), dass die PUI neben einer laseraktivierten Spülung die effektivste Auflösung der Schmierschicht erreicht. Ähnliches wurde von Jiang et al. (2010) beschrieben, die erörterten, dass eine ultraschallaktivierte Spülung durch die höhere Frequenz das Phänomen des acoustic microstreaming innerhalb der Spülflüssigkeit erzeugt und somit der Schallaktivierung überlegen ist. Duque et al. (2016) wiesen für die PUI eine der Handspülung überlegene Reinigungsleistung beschränkt auf den Isthmus zweier verbundener Wurzelkanäle nach. Die Entfernung von Debris über die gesamte Länge des Wurzelkanals und dadurch die Freilegung von Dentintubuli gelang mit der PUI deutlich besser als mit der Handspülung, obwohl keine vollständige Eliminierung des Debris erreicht wird (Mozo et al. 2014). Andere Studien hingegen zeigten keine Unterschiede zwischen ultraschallaktivierter und schallaktivierter Spülung (Conde et al. 2016). De Castro et al. (2016) bestätigten der PUI eine ähnliche Effektivität wie der Handspülung. Llena et al. (2015) empfahlen zur effektiven Reinigung des Wurzelkanalsystems eine Kombination aus Handspülung während der initialen Präparationsphase und einer passiven ultraschallaktivierten Spülung als Abschlussspülung. Probleme der ultraschallaktivierten Spülung können ein erhöhter Dentinabtrag entlang der Kanalwände sein (Jiang et al. 2010) oder aber die Gefahr der Stufenbildung in gekrümmten Kanälen (Lumley et al. 1992).

\subsubsection{EDDY®}

Der Name EDDY® leitet sich vom englischen eddy ab, was mit Wirbel oder Strudel übersetzt werden kann. Entwickelt wurde EDDY® von Dr. Winfried Zeppenfeld aus Flensburg, der Vertrieb in Deutschland erfolgt durch die Firma VDW aus München. EDDY® ist eine schallaktivierbare, oszillierende Spülspitze aus Polyamid. Die Spitze ist als Einmalprodukt konzipiert und liegt 
in einer Einheitsgröße vor. Sie besitzt ein $3 \mathrm{~mm}$ langes Gewinde, über das sie einfach an vorhandene kompatible Airscaler angeschlossen werden kann. Die Gesamtlänge beträgt $28 \mathrm{~mm}$, der Durchmesser an der Spitze 0,2 mm. Zusätzliche Längenmarkierungen zwischen 18 und $28 \mathrm{~mm}$ erleichtern die Kontrolle der Eindringtiefe. EDDY® besitzt aufgrund der Fertigung aus Polyamid ein sehr geringes Bruchrisiko, erlaubt eine dentinschonende Arbeitsweise und lässt sich auch in stark gekrümmten Wurzelkanälen ohne die Gefahr einer Stufenbildung anwenden. Der Einsatz von EDDY® erfolgt mit Hilfe von Airscalern im Frequenzbereich zwischen 5000 und 6000 Hz. Die dabei entstehenden Schwingungen werden über das Gewinde auf die Spitze übertragen, welche dadurch in oszillierende Schwingungen versetzt wird. Wie bei der PUI kommt es auch bei der Aktivierung durch EDDY® zu einem Kavitationseffekt, bei dem kleinste Gasbläschen entstehen und sich implosionsartig wieder auflösen, wodurch Energie frei wird, die die Reinigungseffektivität verbessern soll. Zusätzlich tritt der Effekt des „acoustic streaming“ auf, dem Entstehen von Wirbelströmen innerhalb der Spülflüssigkeit, die mechanisch eine bessere Reinigung der Wurzelkanalwände gewährleisten sollen.

Die Studienlage zu EDDY® ist aufgrund der kurzen Marktverfügbarkeit noch sehr schlecht. Neuhaus et al. (2016) bestätigten, dass die schallaktivierte Spülung mit EDDY® eine wirksame Methode zur Entfernung von Bakterien aus dem Wurzelkanalsystem darstellt. Wird EDDY® mit $6000 \mathrm{~Hz}$ aktiviert, so ist bei Verwendung einer nicht desinfizierenden Spüllösung die Reinigungsleistung wirksamer als bei der PUI (Neuhaus et al. 2016). Im

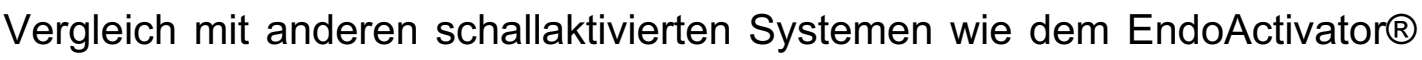
ermittelten Neuhaus et al. eine deutlich bessere Reduktion von Mikroorganismen, was sie mit der viel höheren Frequenz (EDDY®: $6000 \mathrm{~Hz}$, EndoActivator®: max. $170 \mathrm{~Hz}$ ) begründeten. Weiterhin erläuterten sie, dass die bei der Aktivierung der Spitze entstehenden Effekte anderer Natur seien als bei der Ultraschallaktivierung, was durch eine größere Amplitude während der Schwingung erklärt wird. Die Gefahr des unkontrollierten Dentinabtrags bei passiver Ultraschallaktivierung (Retsas et al. 2016) ist bei EDDY® aufgrund der Fertigung aus Polyamid wahrscheinlich geringer. Conde et al. (2016) untersuchten die Effektivität der Reinigung mit EDDY® im Vergleich 
mit PUI, EndoActivator $®$ und Handspülung. Dabei fanden sie keine signifikanten Unterschiede zwischen den aktivierten Gruppen.

\subsection{Wurzelkanaldesinfektion: Spüllösungen}

\subsubsection{Natriumhypochlorit}

Natriumhypochlorit $(\mathrm{NaOCl})$ ist die am gründlichsten untersuchte und am häufigsten eingesetzte Spüllösung zur Desinfektion des Wurzelkanalsystems und gilt heute als Mittel der Wahl. Auch unter dem Trivialnamen „Eau de Labarraque“ bekannt, hat Natriumhypochlorit bereits eine über 200jährige Geschichte hinter sich, die ihre Ursprünge bei dem französischen Chemiker und Apotheker Antoine Germain Labarraque (1777-1850) findet. Dieser entdeckte die Verbindung und deren Eigenschaften im Jahr 1820. Zur damaligen Zeit wurde das Mittel vorwiegend zur Desinfektion und als Bleichmittel verwendet, fand aber auch Anwendung zur Desodorierung von Leichenhallen und Kanalisationen und wurde häufig in Krankenhäusern oder Lazaretten eingesetzt. Chemisch ist $\mathrm{NaOCl}$ ist das Salz der hypochlorigen Säure und besteht aus einer Verbindung aus einem Natriumkation $\left(\mathrm{Na}^{+}\right)$und einem Hypochloritanion $\left(\mathrm{OCl}^{-}\right)$. Es liegt meist in einer leicht gelblichen Lösung vor und riecht deutlich nach Chlor. Der $\mathrm{pH}$-Wert liegt im stark basischen Bereich bei 11-12. Die Anwendung in der Endodontie gründet auf den Eigenschaften von $\mathrm{NaOCl}$, die es bei der Desinfektion des Wurzelkanalsystems zeigt. Bei einer geringen Toxizität ermöglicht es eine effektive Auflösung sowohl von vitalem als auch nekrotischem Gewebe. Es löst dabei organische Bestandteile sowohl des Dentins als auch die organischen Komponenten des smear layers (Gutiérrez et al. 1990; Naenni et al. 2004). Es besitzt eine ausgezeichnete antibakterielle Wirkung gegen die meisten endodontisch relevanten Mikroorganismen und ist zusätzlich in der Lage, Zerfallsprodukte dieser Organismen zu inaktivieren, jedoch nicht vollständig (da Silva et al. 2004). Die DGZMK (Deutsche Gesellschaft für Zahn-, Mund- und Kieferheilkunde) empfiehlt in ihrer Leitlinie zur Wurzelkanaldesinfektion die Anwendung von $\mathrm{NaOCl}$ in einer Konzentration zwischen 0,5 und $5 \%$ als Spüllösung der ersten Wahl. In den meisten Fällen findet $\mathrm{NaOCl}$ Anwendung in einer Konzentration zwischen 1 und $3 \%$. 
Unterschiedliche Faktoren beeinflussen die Wirksamkeit von $\mathrm{NaOCI}$ während der Wurzelkanaldesinfektion. Neben der Konzentration der verwendeten Lösung spielen das Volumen, die Einwirkzeit, die Temperatur und nicht zuletzt die Aktivierung der Lösung eine wichtige Rolle. Dabei gelten einerseits das Volumen und andererseits die Einwirkzeit als entscheidende Faktoren zur effektiven Desinfektion (del Carpio-Perochena et al. 2015). So empfehlen Yamada et al. (1983) ein Volumen von mindestens $10-20 \mathrm{ml}$ pro Kanal. Schäfer (2007) greift dies in seiner Empfehlung für sein Spülprotokoll auf. Ein hohes Spülvolumen sei nötig, da bereits nach etwa zwei Minuten Kontakt mit organischen Geweben die gewebeauflösende Wirkung des $\mathrm{NaOCl}$ deutlich nachlässt (Moorer und Wesselink 1982; Clarkson et al. 2006). Die empfohlene Einwirkzeit sollte mindestens 30-60 Minuten betragen, um eine ausreichende Entfernung des Biofilms zu gewährleisten (Gomes et al. 2001; Sen et al. 1999; Spratt et al. 2001). Dabei ist die Einwirkzeit auch abhängig von der Zusammensetzung des endodontischen Biofilms und der Anatomie des Wurzelkanalsystems (Spratt et al. 2001). Über die zu verwendende Konzentration des $\mathrm{NaOCl}$ besteht kein Konsens. Generell werden Konzentrationen zwischen 0,5 und 5,25 \% diskutiert. Haapasalo et al. (2005) empfehlen eine Konzentration von maximal 0,5 - 1 \%. Im Gegensatz dazu beschrieben Frough-Reyhani et al. (2016), dass eine Konzentration von 1 \% nicht in der Lage sei, eine ausreichende Entfernung des endodontischen Biofilms zu gewährleisten. Sie empfehlen eine Konzentration von 2,5 \%, da diese Konzentration sowohl eine ausreichend geringe Toxizität als auch eine effektive Auflösung des Biofilms gewährleisten kann. Ebenfalls eine Konzentration von 2,5 \% empfehlen Gulsahi et al. (2014), Tanomaru-Filho et al. (2015) und Del Carpio-Perochena et al. (2015), da diese Konzentration die höchste Reduzierung von Biofilmmasse und den effektivsten antibakteriellen Effekt erzielt. Die Verwendung einer Konzentration von 5,25 \% bietet keinen Vorteil gegenüber der 2,5 \% Konzentration (Frough-Reyhani et al. 2016). Dem widersprechen Wang et al. (2015), die einer Konzentration von 5,25 \% einen signifikant höheren antibakteriellen Effekt zusprechen als niedrigeren Konzentrationen. Zu dem gleichen Ergebnis kommen Retamozo et al. (2010), die als effektivste Spülung eine Konzentration von 5,25 \% bei einer Einwirkzeit von 40 Minuten empfehlen. Studien, die noch höhere 
Konzentrationen untersuchten, finden sich seltener. Unter anderem zeigten Stojicic et al. (2010), dass die effektivste Desinfektion mit einer Konzentration von $5,8 \%$ gelingt. Studien von Pashley et al. (1985) und Chang et al. (2001) zeigten, dass eine steigende Konzentration von $\mathrm{NaOCl}$ mit einer steigenden Zytotoxizität einhergeht. Die Temperaturerhöhung einer $\mathrm{NaOCl}$-Lösung kann die Eigenschaften der Lösung beeinflussen. Eine Erwärmung der Lösung auf $37^{\circ} \mathrm{C}$ verbessert die Wirkung gegen Pilze, wie z. B. Candida albicans, jedoch ist die Wirkung bei einer Temperatur von $25^{\circ} \mathrm{C}$ effektiver gegen $E$. faecalis (Gulsahi et al. 2014). Lösungen, die auf $45^{\circ} \mathrm{C}$ erwärmt werden, bieten einen besseren antibakteriellen Effekt, als Lösungen bei $20^{\circ} \mathrm{C}$ (Giardino et al. 2016). Auch Sirtes et al. (2005) kamen zu dem Ergebnis, dass eine Erwärmung auf bis zu $60^{\circ} \mathrm{C}$ eine verbesserte Desinfektion gewährleistet. Im Widerspruch dazu stehen die Ergebnisse von Del Carpio-Perochena et al. (2015), die keinen Unterschied in der Wirksamkeit von $\mathrm{NaOCl}$ bei einer Erwärmung auf $37{ }^{\circ} \mathrm{C}$ feststellten, sondern einen erhöhten antibakteriellen Effekt einer erhöhten Konzentration und einer verlängerten Einwirkzeit zusprachen. Gegenstand weiterer Studien war die Diskussion, welche Auswirkungen die Spülung des Wurzelkanals mit $\mathrm{NaOCl}$ auf die mechanischen Eigenschaften des Dentins hat. So verglichen Ari und Erdemir (2005) die Wirkung von 5,25 $\% \mathrm{NaOCl}$ mit der anderer Lösungen wie EDTA, $\mathrm{CHX}$ und $\mathrm{H}_{2} \mathrm{O}_{2}$ und stellten fest, dass $\mathrm{NaOCl}$ die einzige Spülflüssigkeit ist, die keine signifikanten Veränderungen der mechanischen Eigenschaften des Wurzelkanaldentins verursacht. Im Gegensatz dazu steht eine frühere Studie, welche $\mathrm{NaOCl}$ eine deutliche Reduzierung der Mikrohärte des Dentins bescheinigte (Ari et al. 2004). Andere Studien bestätigten dieses Ergebnis (Aslantas et al. 2014; Garcia et al. 2013; Oliveira et al. 2007; Zaparolli et al. 2012).

\subsubsection{Chelatoren/EDTA}

Der am häufigsten in der Endodontie eingesetzte Chelator ist EDTA (Ethylendiamintetraessigsäure). EDTA ist ein sechszähniger Komplexbildner und kann somit sechsfach an ein Kation binden. Diese Eigenschaft befähigt es dazu, stabile Verbindungen mit Kalziumionen aus dem Dentin einzugehen (Hülsmann et al. 2003), die selbst nur eine geringe Tendenz zur Bindung aufweisen. EDTA ergänzt somit die Spülung mit $\mathrm{NaOCl}$ sinnvoll, da es die 
Löslichkeit der im Wurzelkanalsystem vorliegenden Bestandteile um eine anorganische Komponente erweitert. Durch die Bindung an die anorganischen Bestandteile des Dentins ermöglicht EDTA eine effektive Entfernung der Schmierschicht (Haapasalo et al. 2005; Zehnder et al. 2005). EDTA besitzt selbst keine gewebeauflösenden und nur geringe antimikrobielle Eigenschaften (Haapasalo et al. 2005; Yamaguchi et al. 1996) und sollte daher nur ergänzend eingesetzt werden. In der Endodontie kommt es in der Regel in Konzentrationen zwischen 15 und 20 \% zum Einsatz. Im Zusammenspiel mit $\mathrm{NaOCl}$ ist darauf zu achten, dass EDTA die gewebeauflösende Wirkung von $\mathrm{NaOCl}$ nahezu vollständig inhibiert. So weisen Grawehr et al. (2003) nach, dass bei einer Mischung beider Lösungen EDTA seine Eigenschaften beibehält, $\mathrm{NaOCl}$ jedoch seine gewebeauflösenden Eigenschaften verliert. Diese Ergebnisse bestätigen Zehnder et al. (2005). Sie empfehlen daher, entweder beide Lösungen separat einzusetzen oder bei alternierender Spülung eine höhere Menge an $\mathrm{NaOCl} z u$ verwenden. Tartari et al. (2017) zeigten hingegen, dass eine Mischung beider Substanzen funktionieren kann. Dazu verwendeten sie eine Mischung aus $\mathrm{NaOCl}$ und EDTANa . Bei dieser Mischung beobachteten sie zwar einen langsamen und konzentrationsabhängigen Rückgang von freien Chlormolekülen in der Lösung, aber die Gewebeauflösung und die Entfernung der Schmierschicht wurden positiv beeinflusst. Die Mischung der Substanzen sollte unmittelbar vor deren Anwendung stattfinden (Tartari et al. 2017). Wurzelkanalspülungen, die beide Lösungen einsetzen, gelten heute als vielversprechende Methode zur Desinfektion des Wurzelkanalsystems. Diese Methode beschreibt Schäfer (2007) in seiner Empfehlung eines Spülprotokolls, bei dem EDTA in der Abschlussspülung eingesetzt wird, gefolgt von einer erneuten Spülung mit $\mathrm{NaOCl}$. Auch andere Autoren empfehlen eine kombinierte Spülung (Baumgartner und Mader 1987, Yamada et al. 1983), da diese eine effektivere Desinfektion erlaubt als eine Spülung alleine mit $\mathrm{NaOCl}$. Die erhöhte Effektivität kommt dadurch zu Stande, dass durch die Anwendung von EDTA nachfolgenden desinfizierenden Lösungen wie $\mathrm{NaOCl}$ ein besserer Zugang zu tiefer liegenden Dentinschichten ermöglicht wird (Ørstavik und Haapasalo 1990). Goldberg et al. (1986) zeigten, dass eine Anwendung von EDTA mehr gefüllte Seitenkanäle zur 
Folge hatte. Auch die Dichtigkeit von Wurzelkanalfüllungen wird durch die Anwendung von EDTA verbessert (Behrend et al. 1996, Wennberg und Ørstavik 1990). Wie bei anderen Spüllösungen wird die ideale Einwirkzeit auch bei EDTA diskutiert. Goldberg und Spielberg (1982) zeigten, dass die beste Wirkung bei einer Einwirkzeit von 15 Minuten eintritt. Eine Verlängerung der Einwirkzeit auf 30 Minuten bringt dabei keine Vorteile. Die Studie von Teixeira et al. (2005) wies nach, dass bereits Einwirkzeiten von 1 bis 5 Minuten für eine effektive Entfernung des smear layer ausreichen. Zu diesem Ergebnis kamen auch Scelza et al. (2004), die bei einer Einwirkzeit von 3 Minuten die höchste Anzahl an freien geöffneten Dentinkanälchen nachwiesen. Eine Verlängerung der Einwirkzeit auf 15 Minuten zeigte auch hier keine Vorteile. Eine sehr kurze Einwirkzeit von 1 Minute empfehlen Calt und Serper (2002) und raten von einer Verlängerung der Einwirkzeit ab, da bereits ab 10 Minuten deutliche Dentinerosionen zu beobachten seien. Generelle Zusammenhänge zwischen der Einwirkzeit und der Entmineralisierung des Wurzelkanaldentins beschrieben Hülsmann et al. (2002). Zahlreiche Studien belegen, dass EDTA die Mikrohärte des Wurzelkanaldentins herabsetzt (Ari et al. 2004; Aslantas et al. 2014; CruzFilho et al. 2011; Sen et al. 2009; Turk et al. 2015; Zaparolli et al. 2012). Dabei werden die Verringerung der mechanischen Eigenschaften zum einen der Konzentration der Lösung und zum anderen der Einwirkzeit zugeschrieben. Wie auch bei $\mathrm{NaOCl}$ kann die Effektivität der Schmierschichtentfernung mit EDTA durch die Aktivierung der Lösung verbessert werden. 


\section{Ziele der Studie}

Ziel der Studie ist es, die Effektivität des neuen Spülsystems EDDY® in der Entfernung von Debris und smear layer mit der anderer bereits länger erhältlicher Spülsysteme zu vergleichen. Der Vergleich findet zwischen EDDY®, dem EndoActivator ${ }^{\circledR}$, der passiven Ultraschallspülung mit IrriSafe ${ }^{\mathrm{TM}}$ Feilen und der konventionellen dynamischen Handspülung statt. Die Untersuchung soll unter möglichst realistischen Versuchsbedingungen an standardisiert präparierten mesiobukkalen Wurzelkanälen extrahierter humaner Unterkiefermolaren durchgeführt werden. Die Bewertung des verbliebenen Debris und smear layers soll unter dem Rasterelektronenmikroskop mit Hilfe eines bewährten Score-Systems erfolgen.

Die Null-Hypothese lautet, dass zwischen den untersuchten Gruppen kein signifikanter Unterschied bezüglich des an der Wurzelkanalwand verbliebenen Debris und smear layers besteht. 


\section{Material und Methoden}

Die Studie wurde mit Datum vom 8.1.2015 unter der Antragsnummer 9/12/14 von der Ethikkommission der Universitätsmedizin Göttingen (UMG) genehmigt. Die Extraktion der verwendeten Zähne erfolgte alio loco aus Gründen, die in keinem Zusammenhang zur vorliegenden Studie stehen, und die Indikation zur Extraktion wurde durch nicht an der Studie beteiligte Zahnärzte gestellt. Für alle verwendeten Zähne lag eine schriftliche Einwilligung der Patienten zur Verwendung in anonymisierter Form für wissenschaftliche Zwecke vor. 


\subsection{Versuchsübersicht}

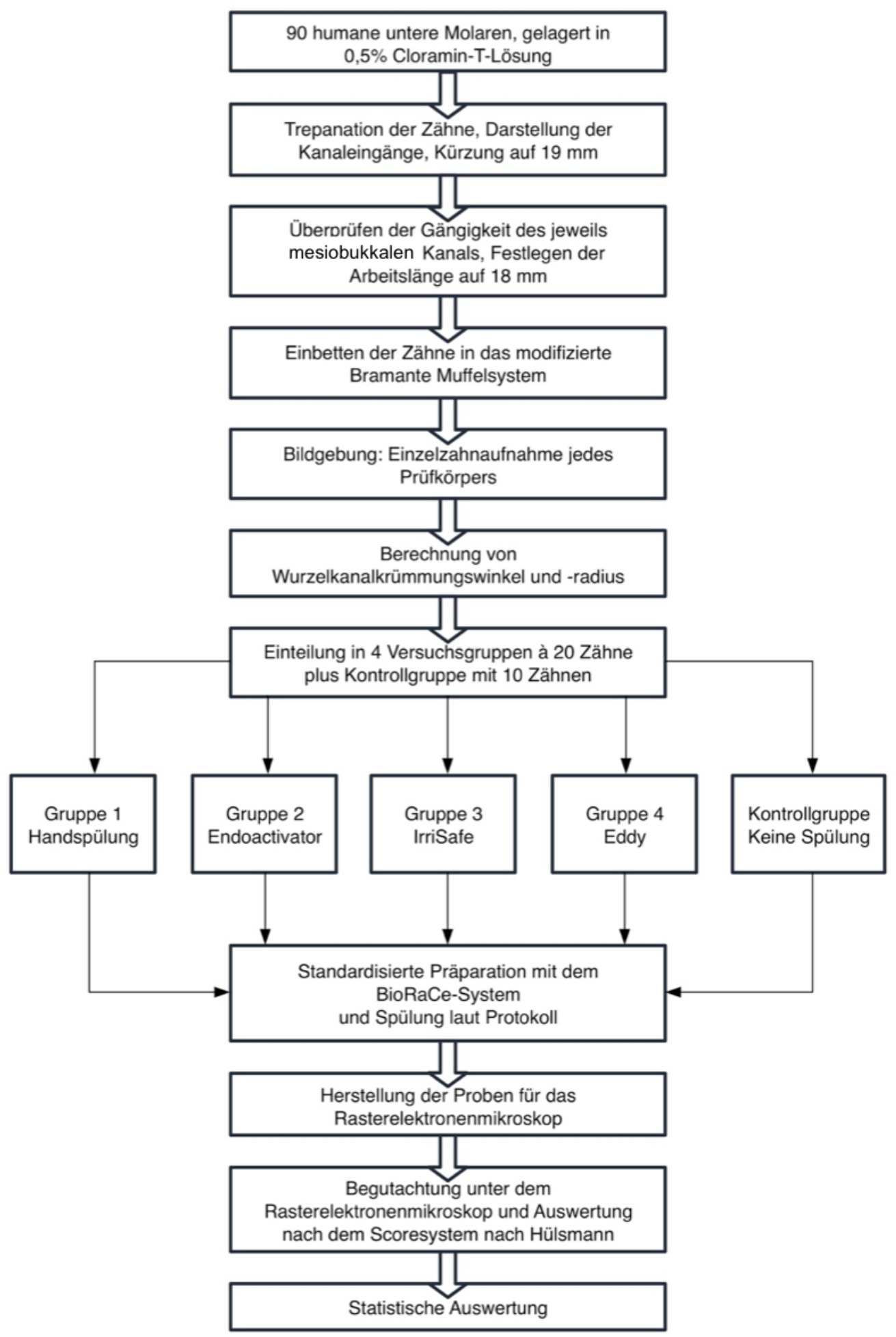

Abbildung 1: Versuchsübersicht 


\subsection{Auswahl der Zähne und vorbereitende Maßnahmen}

Für diese Studie wurden 90 humane Unterkiefermolaren mit intakter Wurzelspitze und einer Krümmung der mesialen Wurzel zwischen 20 und $40^{\circ}$ verwendet. Die Länge der Zähne musste mindestens $19 \mathrm{~mm}$ betragen, um eine Arbeitslänge von $18 \mathrm{~mm}$ zu ermöglichen. Die Wurzelkanäle mussten unbehandelt sein und durften keinen s-förmigen Verlauf aufweisen. Die Zähne sollten keine größeren resorptiven Veränderungen oder kariöse Zerstörungen zeigen. Alle Zähne waren alio loco aus unbekannten Gründen extrahiert worden. Sie wurden von Geweberesten gesäubert und in Chloramin-T-Lösung 0,5 \% gelagert. Zu Beginn erfolgte die Präparation der koronalen Zugangskavität mit einem grob diamantierten zylindrischen Schleifer (Komet Dental, Lemgo, Deutschland) bei 40.000 Umin $^{-1}$. Dabei erfolgten der Abtrag des gesamten Pulpadaches und die Darstellung aller Kanaleingänge. Eventuell vorhandene Dentikel im Pulpakavum wurden ebenfalls entfernt. Im Anschluss wurde die Gängigkeit des mesiobukkalen Wurzelkanals mit Hilfe eines Reamers der ISO-Größe 10 (Orbis Dental, Münster, Deutschland) überprüft. Die Spitze des Reamers sollte dabei gerade am Foramen apicale der Wurzel zu erkennen sein. Um eine einheitliche Arbeitslänge von $18 \mathrm{~mm}$ zu erreichen, wurden die Zähne mit Zirkel und einem Geodreieck (Pelikan AG, Berlin, Deutschland) vermessen und die Zahnlänge auf $19 \mathrm{~mm}$ vereinheitlicht. Zähne, die länger als $19 \mathrm{~mm}$ waren, wurden im koronalen Bereich mit Hilfe einer diamantierten Trennscheibe (Komet Dental, Lemgo, Deutschland) waagerecht zur Zahnachse gekürzt.

\subsection{Herstellen der Versuchskörper}

\subsubsection{Das Muffelsystem}

Für die Studie wurde das Bramante-Muffelsystem verwendet. Das System wurde erstmals 1987 von Bramante et al. beschrieben (Bramante et al. 1987) und in den folgenden Jahren von Calhoun \& Montgomery (1988), McCann et al. (1990) und Hülsmann (1998) modifiziert. In der hier vorliegenden modifizierten Version besteht das Muffelsystem aus drei miteinander verschraubbaren Messingblöcken. Der mittlere Messingblock weist einen 
Hohlraum zur Aufnahme der Prüfobjekte auf. In die Seitenwände des Hohlraumes eingefräste Rillen ermöglichen jederzeit eine exakte Repositionierung der hergestellten Prüfkörper. Die Verschraubung erfolgt über drei Gewindeschrauben mit gegenschraubbarem Endstück. Zur räumlichen Orientierung dienen Einkerbungen an beiden Seitenwänden des Hohlraumes (Abb. 2 und 3).

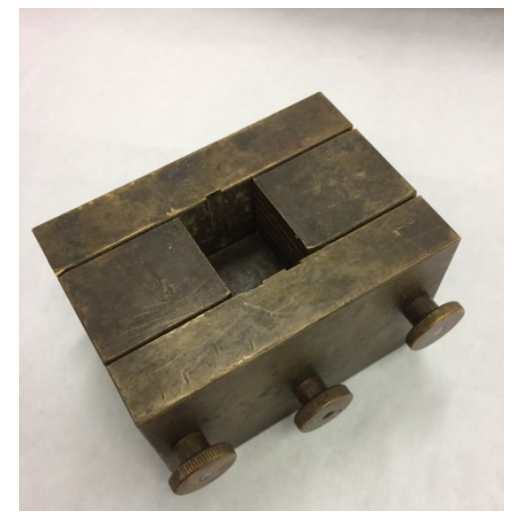

Abbildung 2: BramanteMuffelsystem geschlossen

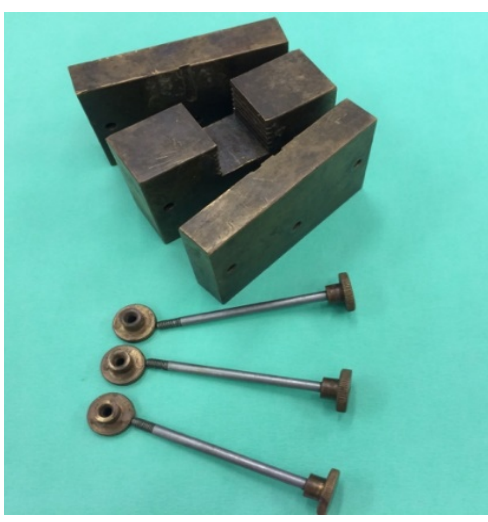

Abbildung 3: BramanteMuffelsystem geöffnet

\subsubsection{Vorbereitende Maßnahmen}

Um die Höhe des Hohlraumes an die Zahnlänge anzupassen, wurde in die unterste Rille des Mittelblocks eine Aluminiumplatte eingeschoben und die Muffel mit Hilfe der Querverschraubungen fest verschlossen. Der Hohlraum wurde mit handelsüblicher Vaseline (weiß, Bombastus-Werke AG, Freital, Deutschland) großzügig isoliert. Um ein Eindringen von Kunststoff in das Wurzelkanalsystem über das Foramen apicale zu verhindern, wurden die Wurzelspitzen der Zähne mit Peripheriewachs (blau, American Dental Systems, Vaterstetten, Deutschland) umschlossen.

\subsubsection{Einbetten der Zähne}

Die Zähne wurden mittig auf der Aluminiumplatte im Hohlraum der Muffel mit Hilfe des die Wurzelspitze umgebenden Peripheriewachses fixiert. Die Bukkalwand des Zahnes zeigte dabei stets zu der Seite der Muffel, die mit zwei Einkerbungen markiert war, um eine spätere Verwechslung 
auszuschließen. Die Schmelz-Zement-Grenze des Zahnes lag auf Höhe der Muffeloberfläche. Der Hohlraum wurde nun mit Prothesenkunststoff (Paladur rosa, Heraeus, Hanau, Deutschland) bis zur Schmelz-Zement-Grenze aufgefüllt. Die Polymerisation erfolgte drucklos für 15 Minuten. Anschließend wurde das Muffelsystem auseinandergeschraubt, und der Prüfkörper konnte durch seitliches Herausschieben aus der Muffel gelöst werden. Jeder Prüfkörper erhielt eine Nummer zur Identifizierung.

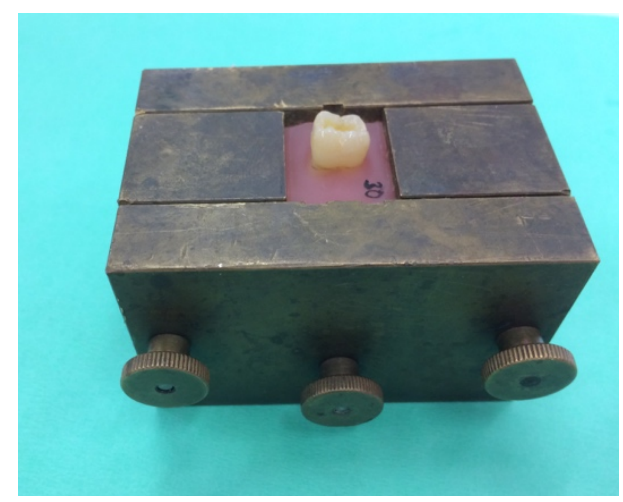

Abbildung 4: In Muffel eingebetteter Zahn

\subsection{Bildgebung}

Von jedem Prüfkörper wurde eine Röntgenaufnahme in Rechtwinkeltechnik erstellt (Sirona Heliodent DS, Belichtungszeit: $23 \mathrm{~ms}$, Sirona, Bensheim, Deutschland) auf Röntgenfilmen von Kodak (Rochester, New York, USA).

\subsubsection{Berechnung von Krümmungswinkel und -radius der Wurzelkanäle}

Die analog entwickelten Röntgenbilder wurden per Durchlichtscanner (CanoScan 9000F, Canon, Tokio, Japan) digitalisiert. Die Bestimmung des Krümmungswinkels erfolgte nach der von Schneider beschriebenen Methode (Schneider 1971). Mit Hilfe eines Bildbearbeitungsprogramms (Photoshop CC 2015, Adobe Incorporated, San José, Kalifornien, USA) wurden zwei Geraden in das Röntgenbild eingezeichnet. Die Gerade I verlief durch den koronalen Kanalanteil. Der Punkt, an dem die Gerade den Kanal verlässt, wurde als $\mathrm{P}$ gekennzeichnet. Eine zweite Gerade II wurde von der Wurzelspitze zu Punkt $P$ eingezeichnet. Der Winkel, der nach apikal von den beiden Geraden gebildet wurde, ist der Krümmungswinkel alpha (Abb. 5). Mit Hilfe des Grafikprogrammes ImageJ (Version 1.50i, Wayne Rasband, National 
Institutes of Health, Rockville, Maryland, USA) wurde der Krümmungswinkel für jeden Prüfkörper bestimmt.

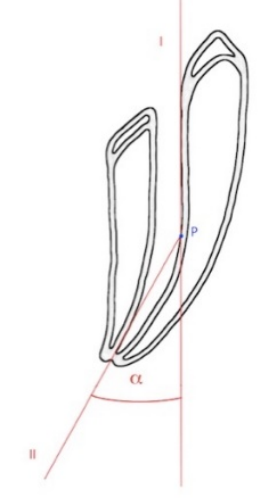

Abbildung 5: Bestimmung des

Krümmungswinkels nach Schneider

Für die Berechnung des Krümmungsradius wurde von einem hypothetischen Kreis und einem gleichschenkligen Dreieck ausgegangen. Die Kreissehne s wurde dabei von den Punkten A und P dargestellt (Zhang und Hu 2010). Der Punkt $A$ befand sich im Röntgenbild $1 \mathrm{~mm}$ oberhalb der Wurzelspitze, der Punkt $P$ beschrieb wie vorhergehend den Punkt, an dem die Gerade I den Wurzelkanal verlässt (Abb. 6). Die Berechnung des Krümmungsradius erfolgte nach der Formel $r=\frac{s}{2 \sin a}$. 


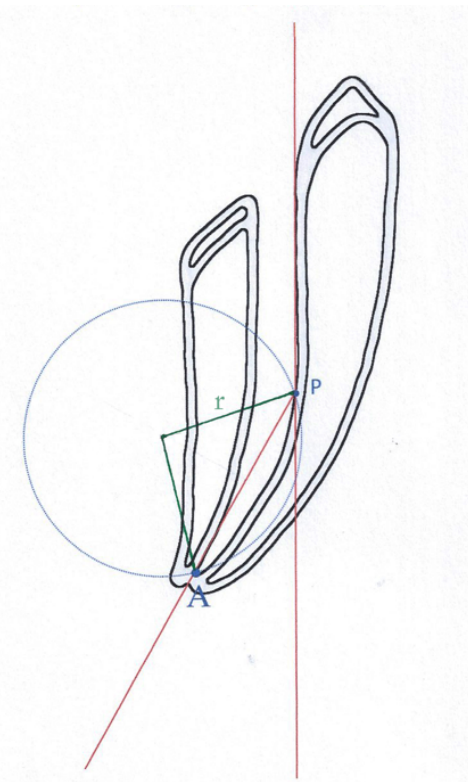

Abbildung 6: Bestimmung des

Krümmungsradius

\subsubsection{Einteilung in die Versuchsgruppen}

Die Einteilung in die Versuchsgruppen erfolgte randomisiert. Anschließend wurden die Mittelwerte der Parameter Wurzelkanalkrümmungswinkel und radius errechnet. Um eine Vergleichbarkeit der Gruppen zu gewährleisten, wurden einzelne Prüfkörper zwischen den Gruppen getauscht, um die Mittelwerte anzunähern. 


\subsection{Präparation der Wurzelkanäle}

\subsubsection{Das BioRaCe ${ }^{T M}$-System}

Das BioRaCe ${ }^{\text {TM }}$-System (FKG Dentaire, La-Chaux des-Fonds, Schweiz) ist ein maschinelles Nickel-Titan-System. Es leitet sich vom RaCe ${ }^{\mathrm{TM}}$-System des gleichen Herstellers ab, weist aber in Hinblick auf Größen, Konizitäten und Arbeitssequenzen Unterschiede zu diesem System auf. „RaCe“ steht für „Reamer with alternating Cutting edges“ (Reamer mit alternierenden Schneidekanten). Das Arbeitsteil der Instrumente besteht abwechselnd aus kurzen gedrehten Abschnitten, die sich mit längeren nicht gedrehten Abschnitten abwechseln. Sinn dieser Geometrie ist es, einen Einschraubeffekt in den Wurzelkanal zu minimieren. Aufgrund des Zusammenspiels der Größen und Konizitäten der Instrumente soll eine ausreichend weite apikale Präparation ermöglicht werden. Weiterhin zeichnen sich die Instrumente des Systems durch einen dreieckigen Querschnitt und eine nicht schneidende Sicherheitsspitze aus. Das BioRaCe $^{\text {TM }}$-System arbeitet mit der Single-Length-Technik, so dass alle Instrumente (mit Ausnahme von BR0) auf Arbeitslänge angewendet werden. Die vom Hersteller empfohlenen Einstellungen für Geschwindigkeit und Drehmoment liegen bei 500-600 $\mathrm{Umin}^{-1}$ und $1 \mathrm{Ncm}$. Das System setzt sich aus einer Basissequenz und einer Extended Sequenz zusammen (Tab. 1). In der vorliegenden Studie wurde ausschließlich die Basissequenz genutzt. 


\begin{tabular}{|c|c|c|c|}
\hline Basissequenz & Größe & Konizität & Besonderheiten \\
\hline BRO $\bigcirc$ & 25 & .08 & $\begin{array}{l}\text { Wird nicht auf Arbeitslänge } \\
\text { angewendet }\end{array}$ \\
\hline BR1 $\bigcirc$ & 15 & .05 & \\
\hline BR2 $\bigcirc$ & 25 & .04 & \\
\hline BR3 & 25 & .06 & $\begin{array}{l}\text { Aufgrund der hohen Konizität soll } \\
\text { BR3 bei starker apikaler Krümmung } \\
\text { nicht auf Arbeitslänge angewendet } \\
\text { werden }\end{array}$ \\
\hline BR4 & 35 & .04 & \\
\hline BR5 & 40 & .04 & \\
\hline \multicolumn{4}{|l|}{$\begin{array}{l}\text { Extended } \\
\text { Sequenz }\end{array}$} \\
\hline BR4C & 35 & .02 & \\
\hline BR5C & 40 & .02 & \\
\hline BR6 $\bigcirc$ & 50 & .04 & \\
\hline BR7 & 60 & .02 & \\
\hline
\end{tabular}

Tabelle 1: Übersicht über die BioRaCe ${ }^{\mathrm{TM}}$-Sequenzen

\subsubsection{Die standardisierte Präparation mit dem BioRaCe ${ }^{\mathrm{TM}}$-System}

Die Präparation mit dem BioRaCe ${ }^{\mathrm{TM}}$-System setzte sich aus zwei Präparationsphasen zusammen. Zunächst erfolgte eine manuelle Präparation, die der Schaffung eines Gleitpfades sowie der Erweiterung des Kanals auf ISO Größe 15 diente, anschließend die rotierende Präparationsphase mit der Basissequenz.

\subsubsection{Manuelle Präparationsphase}

Für die manuelle Präparationsphase wurden Reamer und Hedströmfeilen der ISO-Größen 10 und 15 (VDW, München, Deutschland) verwendet. Nach 
jedem Instrumentenwechsel erfolgte eine Spülung nach Protokoll. Der Wurzelkanal wurde in dieser Phase bis zur ISO-Größe 15 erweitert.

\subsubsection{Rotierende Präparationsphase}

Im Anschluss erfolgte die rotierende Präparationsphase mit der BioRaCe ${ }^{\mathrm{TM}}$ Basissequenz. Dabei wurde folgende Reihenfolge eingehalten:

1. BRO: Mit Hilfe der BR0-Feile wurden der Kanaleingang und der koronale Kanalanteil erweitert. Das Pulpakavum wurde mit $\mathrm{NaOCl}$ gefüllt und das Instrument rotierend in den Kanal eingebracht. Durch vier sanfte Auf- und Abwärtsbewegungen erfolgte die Präparation. Die Spanräume des Instrumentes wurden manuell gereinigt und der Vorgang wiederholt, bis etwa $6 \mathrm{~mm}$ des koronalen Kanalanteils bearbeitet waren. Anschließend erfolgten eine Spülung nach Protokoll und die manuelle Rekapitulation der Arbeitslänge mit einem Reamer der Größe 10.

2. BR1 bis BR5: Die nachfolgenden Instrumente wurden rotierend in den mit Spülflüssigkeit gefüllten Kanal auf Arbeitslänge eingebracht und durch vier bis sechs leicht pumpende Bewegungen angewendet. Nach jedem Instrumentenwechsel erfolgte die Spülung laut Protokoll.

3. Abschlussspülung 


\subsection{Versuchsgruppen und Spülprotokolle}

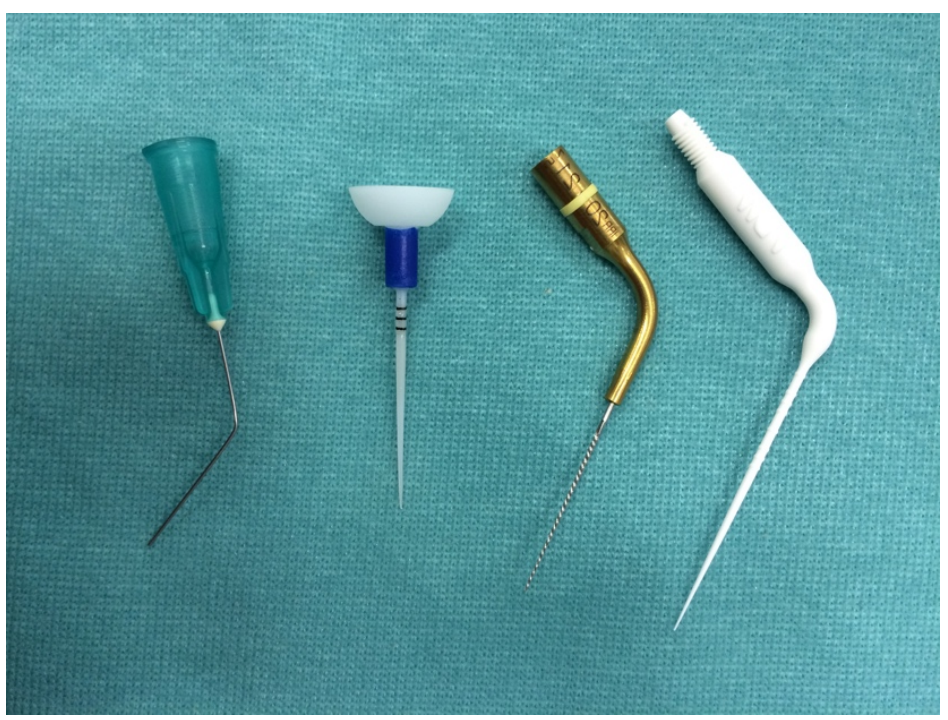

Abbildung 7: Von links nach rechts: Gruppe 1: Endoneedle für Handspülung, Gruppe 2: EndoActivator®, Gruppe 3: IrriSafe ${ }^{\mathrm{TM}}$, Gruppe 4: EDDY®

\subsubsection{Gruppe 1 Spülprotokoll: Handspülung}

Für die manuelle Spülung wurden eine zweiteilige Kunststoffspritze (5 ml, B. Braun, Melsungen, Deutschland) sowie eine Spülspitze (VMK Endoneedle nach Dr. J. Buquet, Vedefar NV, Dilbeek, Belgien) mit einem Außendurchmesser von 0,35 mm und einem Innendurchmesser zwischen 0,17 und $0,19 \mathrm{~mm}$ verwendet.

\section{Spülprotokoll:}

- Nach jedem Bearbeitungsgang erfolgte die manuelle Zufuhr von $2 \mathrm{ml}$ $\mathrm{NaOCl} 3 \%$ über $20 \mathrm{~s}$ mit etwa zehn leichten vertikal pumpenden Bewegungen. Gleichzeitig erfolgte ein Absaugen der koronal austretenden Spülflüssigkeit.

- Abschlussspülung: In drei Zyklen wurden je $2 \mathrm{ml}$ Spüllösung nach obigem Vorgehen appliziert.

- Die maximale Eindringtiefe der Spülspitze lag während der Abschlussspülung bei $17 \mathrm{~mm}$.

\subsubsection{Gruppe 2 Spülprotokoll: EndoActivator ${ }^{\circledR}$}

Für die Prüfkörper der Gruppe 2 wurde das Gerät EndoActivator® verwendet mit einer Spülspitze der Größe „Large“ (35/.04). 
Spülprotokoll:

- Nach jedem Bearbeitungsgang wurden $2 \mathrm{ml} \mathrm{NaOCl} 3 \%$ manuell zugeführt und mit dem EndoActivator® für $20 \mathrm{~s}$ aktiviert.

- Abschlussspülung: Drei Spülzyklen nach obigem Vorgehen. Die Aktivierung wurde bei $10.000 \mathrm{cpm}$ (Counts per minute) durchgeführt. Zusätzlich wurden 10 leichte Vertikalbewegungen von etwa 2-3 mm pro Zyklus ausgeführt.

- Die maximale Eindringtiefe der EndoActivator®-Spitze lag bei $17 \mathrm{~mm}$.

\subsubsection{Gruppe 3 Spülprotokoll: IrriSafe ${ }^{\mathrm{TM}}$}

Der Einsatz der IrriSafe ${ }^{\text {TM }}$ Spülspitze (Größe 20/21) erfolgte mit einem Ultraschall-Mikromotor (VDW Ultra, VDW, München, Deutschland). Die Einstellung des Motors erfolgte mit der vom Hersteller für eine Aktivierung der Spülung empfohlenen Einstellung von 30, was einer Frequenz von etwa 30 $\mathrm{KHz}$ entsprach.

Spülprotokoll:

- Manuelle Zufuhr von $2 \mathrm{ml} \mathrm{NaOCl} 3 \%$ nach jedem Bearbeitungsgang. Aktivierung der Flüssigkeit für $20 \mathrm{~s}$.

- Abschlussspülung: Drei Zyklen nach obigem Vorgehen.

- Die maximale Eindringtiefe der IrriSafe ${ }^{\mathrm{TM}}$ Spitze lag bei $17 \mathrm{~mm}$.

\subsubsection{Gruppe 4 Spülprotokoll: EDDY®}

Die Spülung der Prüfkörper der Gruppe 4 wurde mit Hilfe von EDDY® durchgeführt. Die Spitze wurde dazu in einen Airscaler (Proxeo ZA 55LM, W\&H, Bürmoos, Salzburg, Österreich) eingeschraubt.

\section{Spülprotokoll:}

- Nach jedem Bearbeitungsgang manuelle Zufuhr von $2 \mathrm{ml} \mathrm{NaOCl} 3 \%$ und Aktivierung mit EDDY ${ }^{\circledR}$ für $20 \mathrm{~s}$ bei einer Frequenz von $5000 \mathrm{~Hz}$ und einem Arbeitsdruck von 3 bar.

- Abschlussspülung: Drei aufeinanderfolgende Zyklen nach obigem Beispiel. Zusätzlich wurden 10 leicht pumpende vertikale Bewegungen von etwa 3 $\mathrm{mm}$ im Kanal ausgeführt.

- Nach jedem Zyklus erfolgte die manuelle Spülung mit je $2 \mathrm{ml} \mathrm{NaOCl} 3 \%$

- Die maximale Eindringtiefe des EDDY ${ }^{\circledR}$ lag bei $17 \mathrm{~mm}$.

\subsubsection{Gruppe 5 Kontrollgruppe}

Die Prüfkörper der Kontrollgruppe wurden nach der Präparation nicht gespült. 


\subsection{Herstellung der Proben für das Rasterelektronen- mikroskop}

Für die Untersuchung der Proben unter dem Rasterelektronenmikroskop wurden von jedem Prüfkörper zwei Proben hergestellt, je eine aus dem koronalen und dem apikalen Kanaldrittel. Die Herstellung der Proben wurde in drei Schritten durchgeführt.

\subsubsection{Herstellung der Sägeschnitte}

Im ersten Schritt wurden Querschnitte der einzelnen Prüfkörper hergestellt. Dazu wurden mit Hilfe einer diamantierten Trennscheibe (FinoDisc, Fino, Bad Bocklet, Deutschland) waagerechte Sägeschnitte in einer Dicke von 3-4 mm jeweils im koronalen und apikalen Drittel der Wurzel hergestellt. Um eine Verunreinigung des Kanallumens durch Dentin- oder Kunststoffschleifstaub zu verhindern, wurde zuvor eine Guttaperchaspitze der ISO-Größe 35 (VDW, München, Deutschland) in den Wurzelkanal eingebracht. Im zweiten Schritt erfolgte die Längsspaltung des Kanalsegments. Dazu wurde mit Hilfe einer kleineren diamantierten Trennscheibe (Komet Dental, Lemgo, Deutschland) aus mesialer sowie distaler Richtung jeweils eine Rille bis kurz vor das Kanallumen angelegt, jedoch ohne dieses zu eröffnen. Die auf diese Weise entstandenen Sollbruchstellen wurden anschließend mit Hilfe eines Wachsmessers (Heraeus, Hanau, Deutschland) vorsichtig gebrochen. Auf diese Weise konnte einer Verunreinigung der Kanalwände auch in diesem Arbeitsschritt vorgebeugt werden.

\subsubsection{Herstellung der Nietenproben}

Die so hergestellten Proben wurden im Folgenden auf Aluminiumnieten (DIN $661,3,0 \mathrm{~mm} \times 12,0 \mathrm{~mm}$, Gesipa, Mörfelden-Walldorf, Deutschland) fixiert. Dazu wurde eine geringe Menge lichthärtendes Komposit (Venus, Heraeus, Hanau, Deutschland) auf den oberen Teil der Niete aufgebracht. Die Probe wurde nun so auf dem Kunststoff platziert, dass die bukkale Kanalinnenwand nach oben zeigte und so eine ungehinderte Aufsicht auf diese Wand möglich war. Mit einem Heidemannspatel (Heraeus, Hanau, Deutschland) wurde der Kunststoff vorsichtig um Niete und Probenränder herum modelliert, so dass 
die Probe nicht mehr abfallen konnte. Mit Hilfe einer LED Polymerisationslampe (BA Optima 10, B.A. International, Northhampton, England) wurde der Kunststoff für 20 s gehärtet.

\subsubsection{Bedampfung der Proben}

Die Proben wurden im Feinvakuum-Coater Leica EM ACE200 (Leica Microsystems, Wetzlar, Deutschland) mit einer Platin-Palladium-Schicht bedampft. Die Bedampfungsdauer betrug 185 Sekunden.

\subsubsection{Auswertung unter dem Rasterelektronenmikroskop}

Die Auswertung der mit Nummern versehenen und nach dem Zufallsprinzip sortierten Proben erfolgte jeweils ohne Kenntnis der Gruppenzugehörigkeit der jeweiligen Probe mit Hilfe des Feldemissionsrasterelektronenmikroskops Zeiss Ultra Plus (Carl Zeiss AG, Oberkochen, Deutschland). Die Bewertung des verbliebenen Debris erfolgte unter 200x Vergößerung, die des verbliebenen smear layers unter 1000x Vergrößerung. Die Proben wurden zunächst unter geringer Vergrößerung grob justiert und auf einen zufällig eingestellten Punkt auf der Mitte der Oberfläche des Objektes in der koronalen und anschließend der apikalen Wurzelhälfte fixiert. Erst anschließend wurde die Vergrößerung schrittweise hochgefahren, ohne die Lage des Objektes zu verändern. Dies sollte verhindern, dass das REM immer auf besonders gut oder schlecht aussehende Wandbereiche eingestellt wurde.

\subsection{Bewertungskriterien (Scores)}

Die Bewertung der Proben unter dem Rasterelektronenmikroskop erfolgte nach den fünf Scores nach Hülsmann (1998) zur Beurteilung von Debris und smear layer auf der Kanalinnenwand. 
Die Scores zur Bewertung von Debris lauten:

Score 1: Saubere, glatte Kanalwand oder nur vereinzelte kleine Auflagerungen (Abb. 8)

Score 2: Wenige kleine Debrisinseln (Abb. 9)

Score 3: Zahlreiche Debrisinseln, < 50\% der Kanalwand von Debris bedeckt (Abb. 10)

Score 4: > 50\% der Kanalwand von Debris bedeckt (Abb. 11)

Score 5: Gesamte oder fast gesamte Kanalwand von Debrisschicht bedeckt (Abb. 12)

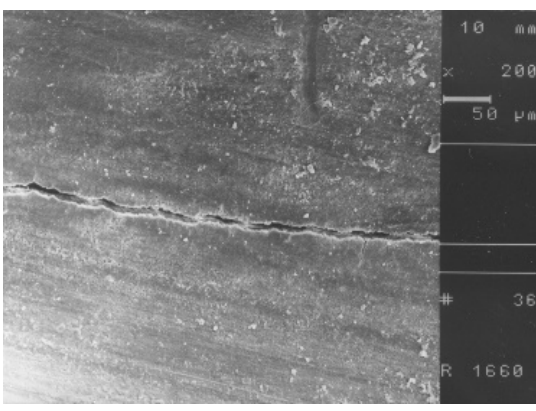

Abbildung 8: Score 1 Debris

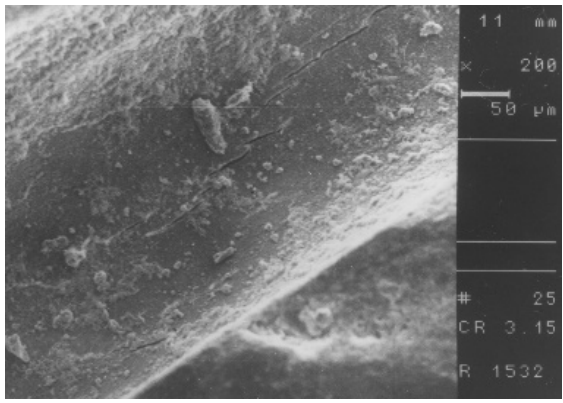

Abbildung 10: Score 3 Debris

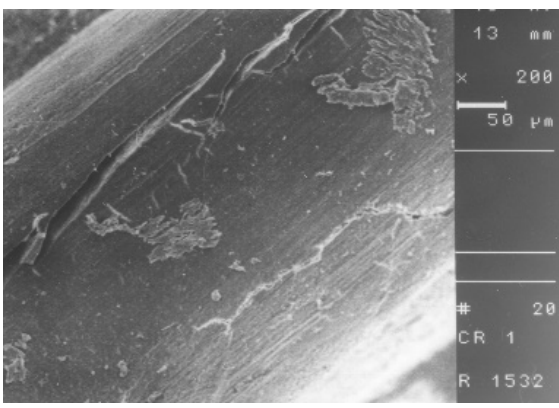

Abbildung 9: Score 2 Debris

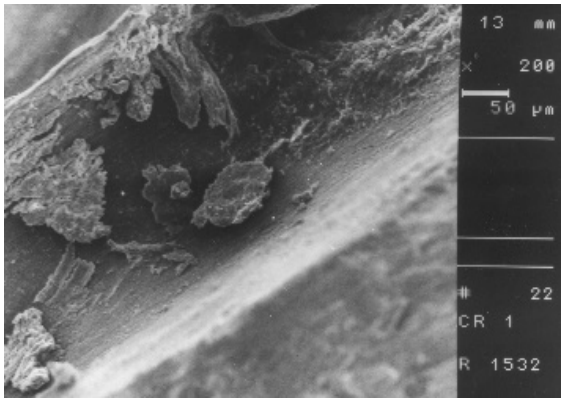

Abbildung 11: Score 4 Debris 


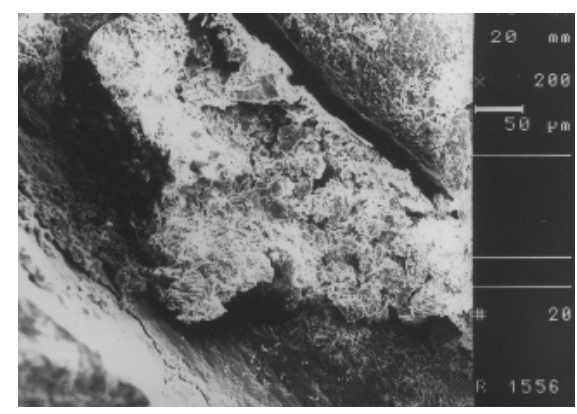

Abbildung 12: Score 5 Debris

Die Scores zur Beurteilung des smear layer lauten:

Score 1: Kein smear layer, überwiegend offene Dentintubuli (Abb. 13)

Score 2: Kleinere Bezirke mit dünnem smear layer, einige offene Dentintubuli (Abb. 14)

Score 3: Homogener smear layer auf der gesamten Kanalwand, nur wenige offene Dentintubuli (Abb. 15)

Score 4: Dichter smear layer auf der gesamten Kanalwand, alle Dentintubuli verblockt (Abb. 16)

Score 5: Dichter, inhomogener smear layer auf der gesamten Kanalwand (Abb. 17)
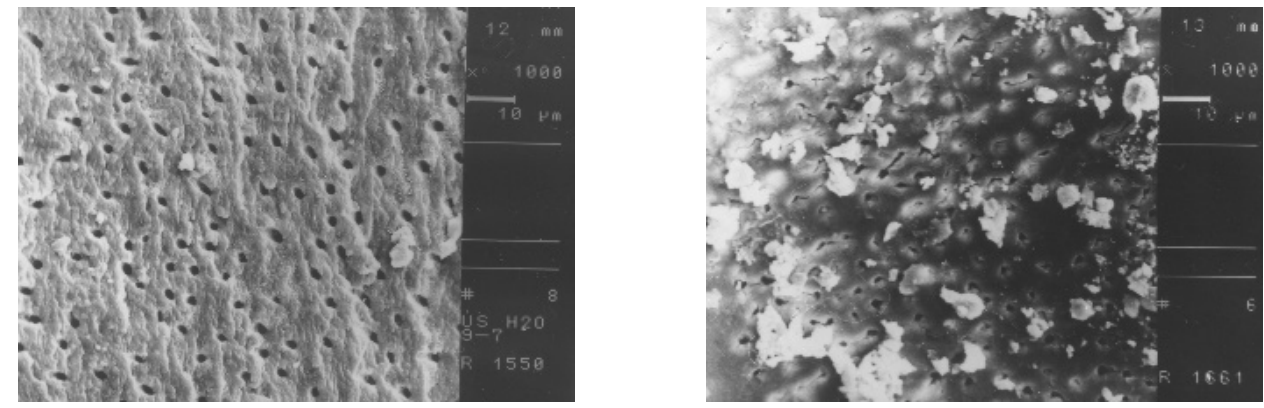

Abbildung 13: Score 1 smear layer Abbildung 14: Score 2 smear layer 

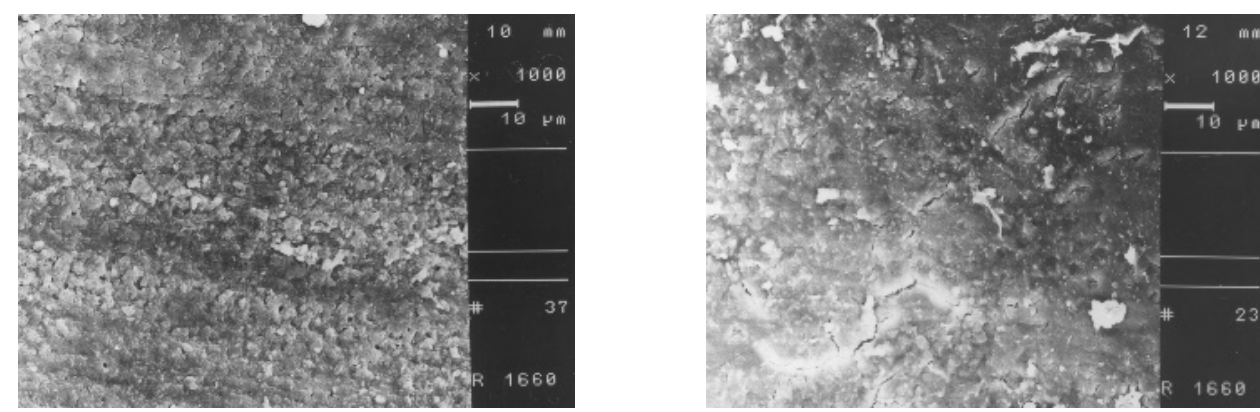

Abbildung 15: Score 3 smear layer

Abbildung 16: Score 4 smear layer

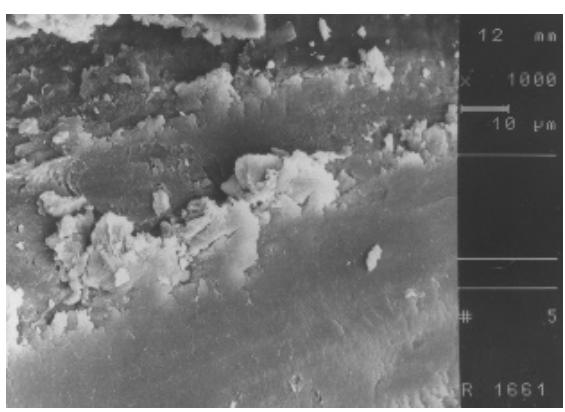

Abbildung 17: Score 5 smear layer 


\section{$5 \quad$ Ergebnisse}

\subsection{Entfernung des Debris: Übersicht}

Die Verteilung der Scores für die Entfernung des Debris nach der rasterelektronenmikroskopischen Untersuchung sind in Tabelle 2 zusammengefasst.

\begin{tabular}{|c|c|c|c|c|c|c|}
\hline $\begin{array}{c}\text { Debris } \\
\text { Score }\end{array}$ & Region & $\begin{array}{c}\text { Gruppe 1 } \\
\text { Hand- } \\
\text { spülung }\end{array}$ & $\begin{array}{c}\text { Gruppe 2 } \\
\text { Endo- } \\
\text { Activator }\end{array}$ & $\begin{array}{c}\text { Gruppe 3 } \\
\text { IrriSafe } \\
\text { PUI }\end{array}$ & $\begin{array}{c}\text { Gruppe 4 } \\
\text { EDDY }\end{array}$ & $\begin{array}{c}\text { Gruppe 5 } \\
\text { Kontrolle }\end{array}$ \\
\hline 1 & koronal & 4 & 7 & 7 & 8 & 2 \\
apikal & 2 & 7 & 5 & 4 & 1 \\
\hline 2 & koronal & 6 & 10 & 7 & 9 & 4 \\
& apikal & 10 & 10 & 10 & 11 & 2 \\
\hline \multirow{2}{*}{3} & koronal & 8 & 1 & 4 & 3 & 0 \\
& apikal & 3 & 2 & 3 & 2 & 1 \\
\hline \multirow{2}{*}{5} & koronal & 1 & 1 & 2 & 0 & 1 \\
& apikal & 3 & 0 & 0 & 1 & 3 \\
& koronal & 1 & 0 & 0 & 0 & 5 \\
\hline
\end{tabular}

Tabelle 2: Verteilung der Scores für Debris nach Region und Gruppe

In Abbildung 18 werden die zusammengefassten Ergebnisse für die apikale und koronale Debris-Score-Verteilung grafisch dargestellt.

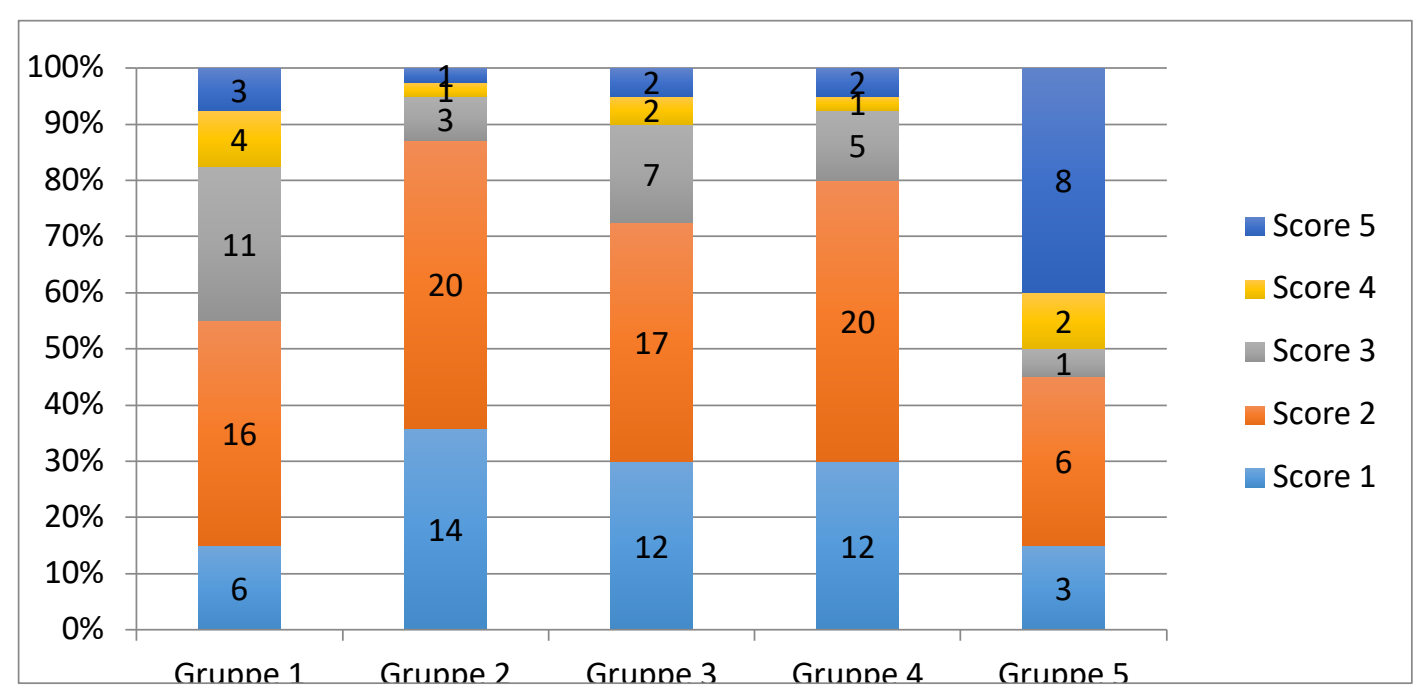

Abbildung 18: Verteilung der Scores für Debris nach Gruppen (1 Handspülung, 2 EndoActivator ${ }^{\circledR}, 3$ IrriSafe $^{\text {TM }}, 4$ EDDY $^{\circledR}, 5$ Kontrollgruppe) 
In Abbildung 18 sind die Werte aus Tabelle 2 zusammengefasst. Es fällt auf, dass die schallaktivierten Gruppen 2 und 4 jeweils zu 87 bzw. 80 \% die Scores 1 und 2 erreichen, was sauberen Wurzelkanalwänden mit vereinzelten Auflagerungen oder kleinen Debrisinseln entspricht. Bei Gruppe 1 sowie Gruppe 3 entfallen nur 55 \% bzw. 72,5\% aller Bewertungen auf diese beiden Scores. Erweitert man die Beurteilung auf die Scores 1 bis 3, erreichen alle Gruppen einen Wert von über $80 \%$. Die schallaktivierten Gruppen 2 und 4 erreichen mit den Scores 1, 2 und 3 sogar 95 \% (EndoActivator®) bzw. 92,5 \% $\left(\right.$ EDDY $\left.^{\circledR}\right)$. Die Scores 4 und 5 (> 50 \% der Kanalwand von Debris bedeckt, bzw. gesamte oder fast gesamte Kanalwand von Debrisschicht bedeckt) treten in Gruppe 1 am häufigsten auf. Zusammenfassend kann festgestellt werden, dass die aktivierten Gruppen der dynamischen Handspülung gegenüber eine bessere Reinigung zeigen.

\subsubsection{Entfernung des Debris im koronalen Wurzelkanalabschnitt}

In Abbildung 19 ist der Box-Plot für die Verteilung der Scores für Debris im koronalen Wurzelkanalanteil dargestellt.

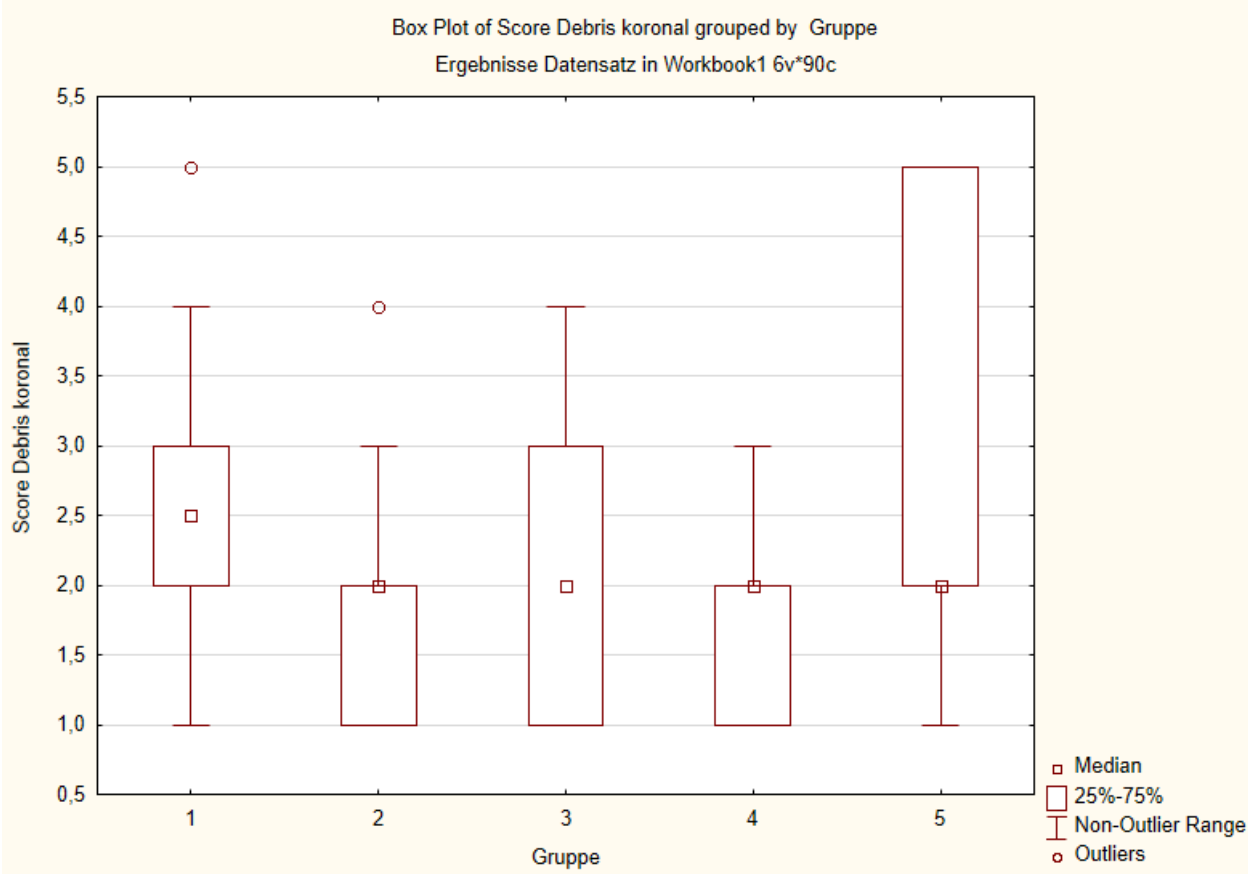

Abbildung 19: Verteilung der Scores für Debris im koronalen Wurzelkanalanteil (1 Handspülung, 2 EndoActivator ${ }^{\circledR}, 3$ IrriSafe $^{\text {TM }}$, 4 Eddy, 5 Kontrollgruppe) 
In Abbildung 19 lässt sich die Verteilung der Scores für Debris für die koronalen Wurzelkanalanteile ablesen. Der Median liegt in den Gruppen 2 bis 5 einheitlich bei einem Score von 2, lediglich in der Gruppe der Handspülung liegt er bei 2,5. Dies bedeutet, dass es sich im koronalen Wurzelkanalabschnitt um überwiegend saubere Kanalwände mit vereinzelten Debrisinseln handelt. Die schallaktivierten Gruppen 2 und 4 (EndoActivator ${ }^{\circledR}$ und $E D D Y^{\circledR}$ ) zeigen ein beinahe identisches Bild. Das Maximum liegt hier bei einem Score von 3, was einer weniger als $50 \%$ von Debris bedeckten Wurzelkanalwand entspricht.

In Abbildung 20 wird die Verteilung der Scores für Debris für die koronalen Wurzelkanalproben grafisch dargestellt.

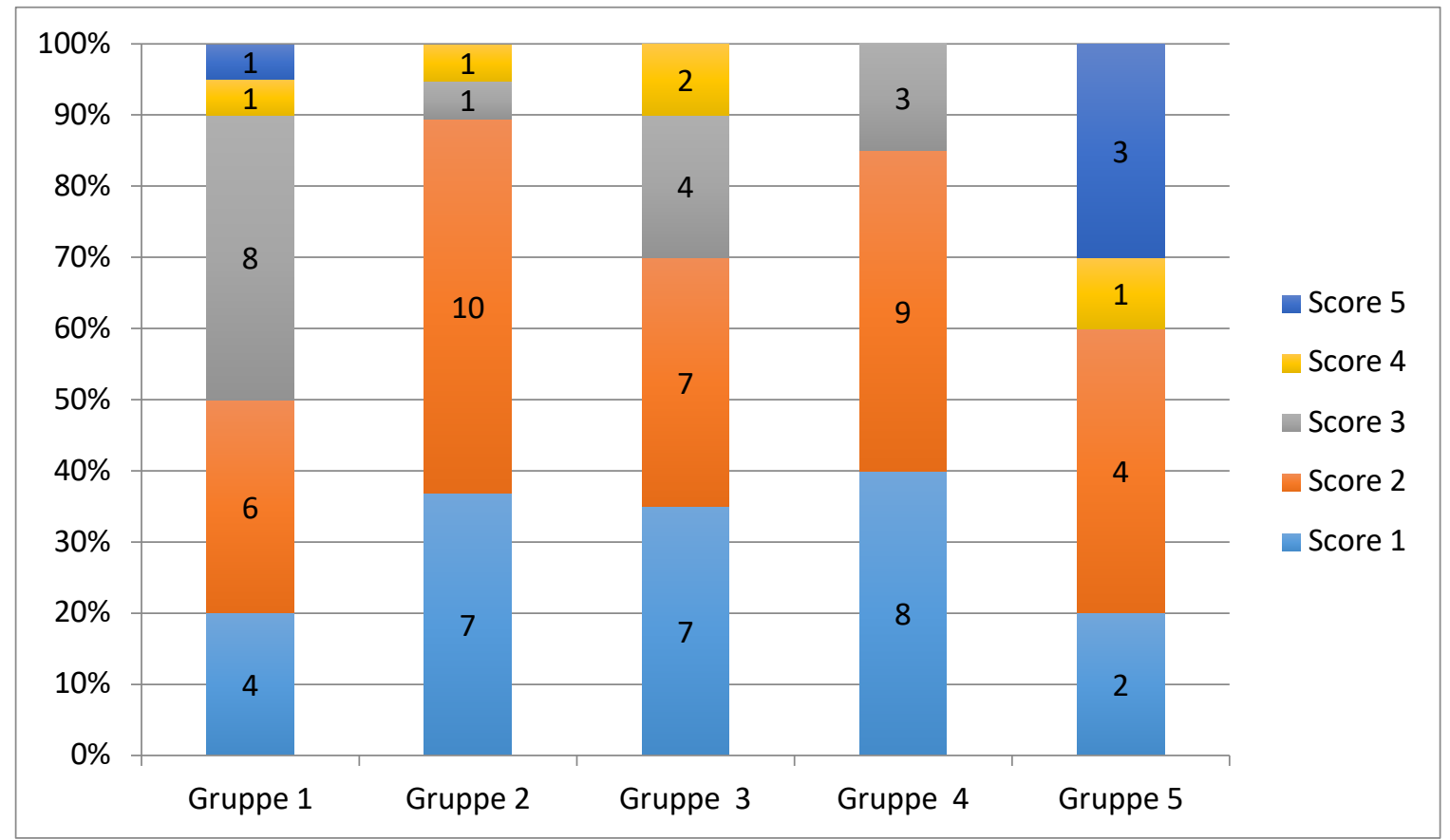

Abbildung 20: Verteilung der Scores für Debris im koronalen Wurzelkanalanteil (1 Handspülung, 2 EndoActivator ${ }^{\circledR}, 3$ IrriSafe $^{\text {TM }}$, 4 EDDY ${ }^{\circledR}$, 5 Kontrollgruppe)

Die aktivierten Gruppen zeigen hier deutlich häufiger die Scores 1 und 2 als die dynamische Handspülung. 


\subsubsection{Entfernung des Debris im apikalen Wurzelkanalabschnitt}

In Abbildung 21 ist der Box-Plot für die Verteilung der Scores für Debris im apikalen Wurzelkanalabschnitt dargestellt.

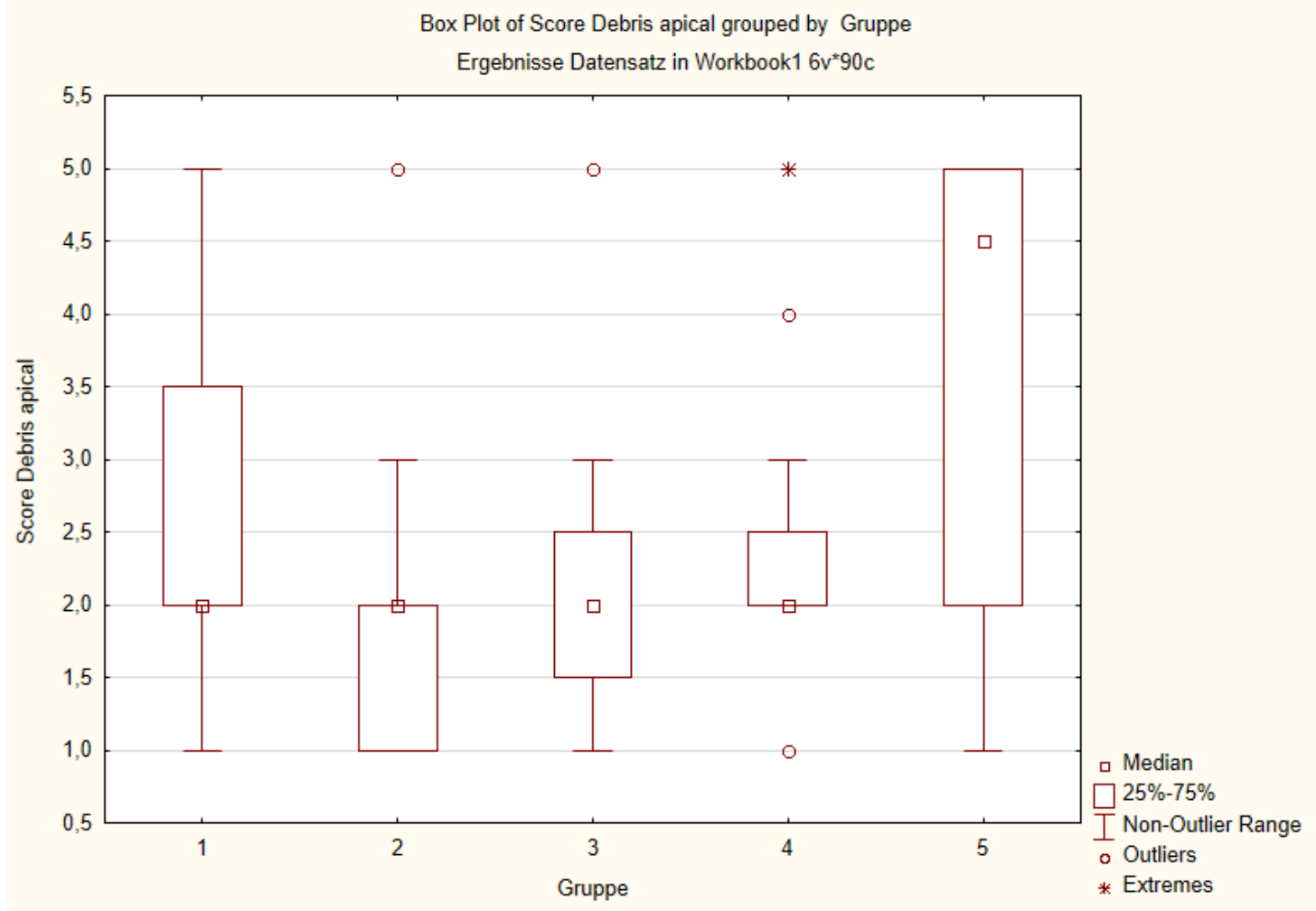

Abbildung 21: Verteilung der Scores für Debris für den apikalen Wurzelkanalanteil (1 Handspülung, 2 EndoActivator ${ }^{\circledR}, 3$ IrriSafe ${ }^{\mathrm{TM}}$, 4 EDDY $^{\circledR}$, 5 Kontrollgruppe) 
In Abbildung 22 wird die Verteilung der Scores für Debris für die apikalen Wurzelkanalproben grafisch dargestellt.

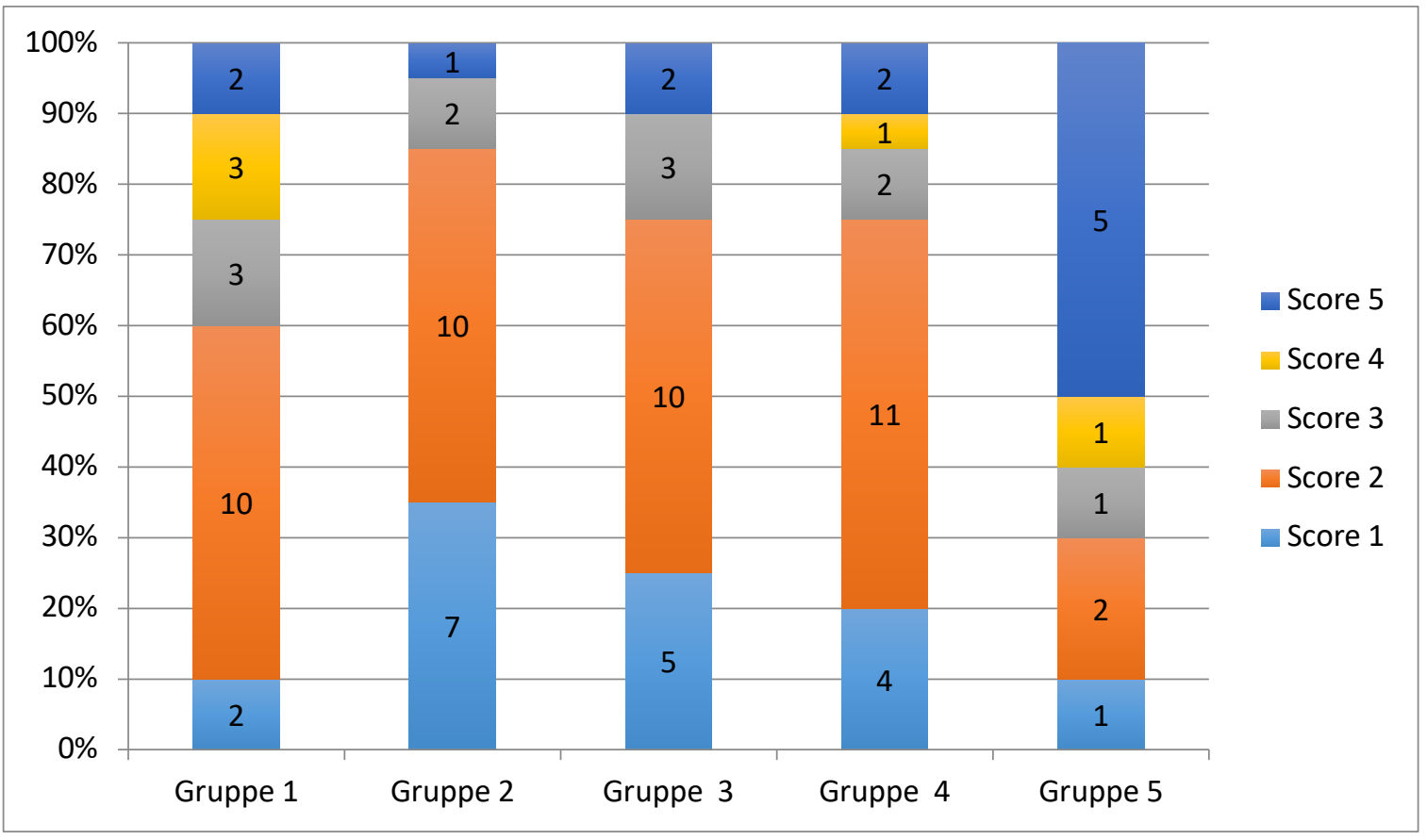

Abbildung 22: Verteilung der Scores für Debris im apikalen Wurzelkanalanteil (1 Handspülung, 2 EndoActivator ${ }^{\circledR}, 3$ IrriSafe $^{\text {TM }}, 4$ EDDY $^{\circledR}, 5$ Kontrollgruppe)

Der EndoActivator ${ }^{\circledR}$ zeigt hier am häufigsten den Score 1. Bei Betrachtung der Scores 1 und 2 kommen die aktivierten Gruppen auf einen Wert von 75 \% (IrriSafe ${ }^{\mathrm{TM}}$ und EDDY ${ }^{\circledR}$ ) bzw. 85 \% (EndoActivator®).

\subsubsection{Vergleich der koronalen und apikalen Reinigungswirkung für Debris}

Betrachtet man die Abbildungen 20 und 22, so wird deutlich, dass im koronalen Wurzelkanalabschnitt bessere Ergebnisse erzielt wurden als im apikalen. Koronal schneiden die schallaktivierten Gruppen am besten ab. So kommt die Gruppe 2 (EndoActivator®) unter Berücksichtigung der Scores 13 auf $95 \%$, die Gruppe 4 (EDDY ${ }^{\circledR}$ ) auf $100 \%$. Die Gruppe 1 (dynamische Handspülung) schnitt koronal am schlechtesten ab. 


\subsection{Entfernung des smear layer: Übersicht}

Die Verteilung der Scores für die Entfernung des smear layer nach der rasterelektronenmikroskopischen Untersuchung ist in Tabelle 3 zusammengefasst.

\begin{tabular}{|c|c|c|c|c|c|c|}
\hline $\begin{array}{c}\text { smear } \\
\text { layer } \\
\text { Score }\end{array}$ & Region & $\begin{array}{c}\text { Gruppe 1 } \\
\text { Handspülung }\end{array}$ & $\begin{array}{c}\text { Gruppe 2 } \\
\text { EndoActivator } \\
\circledR\end{array}$ & $\begin{array}{c}\text { Gruppe 3 } \\
\text { IrriSafe } \\
\text { PUI }\end{array}$ & $\begin{array}{c}\text { Gruppe 4 } \\
\text { EDDY }\end{array}$ & $\begin{array}{c}\text { Gruppe 5 } \\
\text { Kontrolle }\end{array}$ \\
\hline \multirow{2}{*}{1} & koronal & 4 & 1 & 4 & 4 & 2 \\
& apikal & 0 & 1 & 2 & 1 & 0 \\
\hline \multirow{2}{*}{2} & koronal & 2 & 6 & 6 & 8 & 1 \\
& apikal & 1 & 2 & 3 & 1 & 0 \\
\hline \multirow{2}{*}{3} & koronal & 6 & 7 & 5 & 7 & 3 \\
& apikal & 6 & 10 & 8 & 12 & 1 \\
\hline \multirow{2}{*}{4} & koronal & 3 & 5 & 3 & 1 & 1 \\
& apikal & 7 & 4 & 6 & 3 & 4 \\
\hline \multirow{2}{*}{5} & koronal & 5 & 0 & 2 & 0 & 3 \\
& apikal & 6 & 3 & 1 & 3 & 5 \\
\hline
\end{tabular}

Tabelle 3: Verteilung der Scores für smear layer nach Region und Gruppe

In Abbildung 23 werden die zusammengefassten Ergebnisse aus Tabelle 3 für die Verteilung der Scores für smear layer in den koronalen und apikalen Wurzelkanalanteilen grafisch dargestellt.

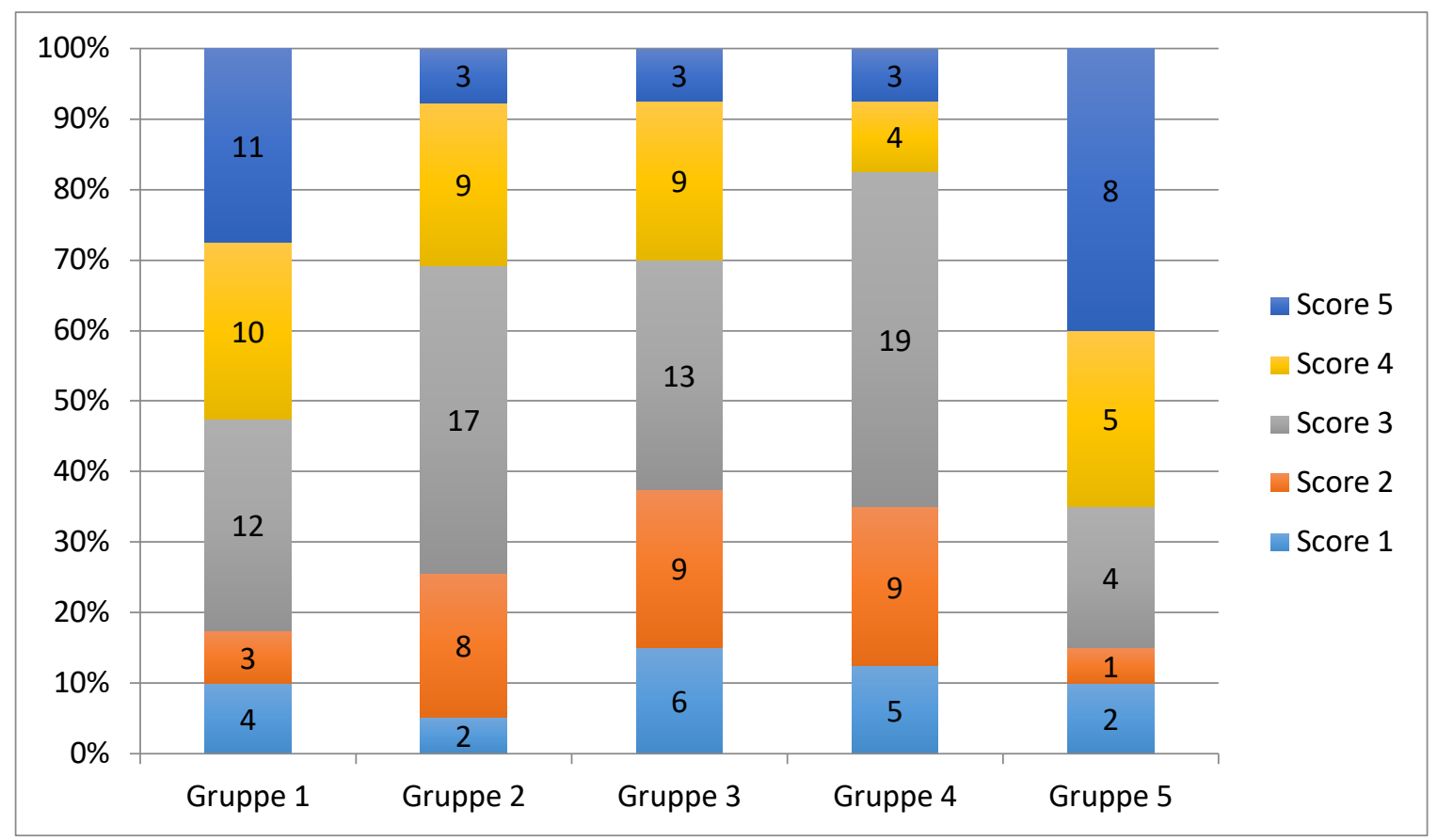

Abbildung 23: Verteilung der Scores für smear layer nach Gruppen. (1 Handspülung, 2 EndoActivator ${ }^{\circledR}, 3$ IrriSafe $^{\mathrm{TM}}$, 4 EDDY ${ }^{\circledR}, 5$ Kontrollgruppe) 
Die Verteilung der Scores für smear layer unterscheidet sich deutlich von der des Debris. Die Scores 1 und 2 wurden deutlich seltener vergeben, die Gewichtung hat sich hier zu den Scores 3 und 4 hin verschoben (Homogener smear layer auf der gesamten Kanalwand, nur wenige offene Dentintubuli, bzw. dichter smear layer auf der gesamten Kanalwand, alle Dentintubuli verblockt). Zieht man die Scores 1 und 2 als Maßstab für eine saubere Wurzelkanalwand heran, so zeigen die Gruppen 3 (IrriSafe ${ }^{\mathrm{TM}}$ ) mit 37,5 \% und $4\left(\right.$ EDDY $\left.^{\circledR}\right)$ mit $35 \%$ die besten Ergebnisse. Insgesamt betrachtet zeigen die aktivierten Gruppen auch hier einen Vorteil gegenüber der dynamischen Handspülung. Die Scores 4 und 5 (Dichter smear layer auf der gesamten Kanalwand, alle Dentintubuli verblockt, bzw. dichter, inhomogener smear layer auf der gesamten Kanalwand) wurden mit 52,5 \% am häufigsten in Gruppe 1 (dynamische Handspülung) und am seltensten in Gruppe 4 $\left(\right.$ EDDY $\left.^{\circledR}\right)$ mit $17,5 \%$ vergeben. 


\subsubsection{Entfernung des smear layer im koronalen Wurzelkanalabschnitt}

In Abbildung 24 ist der Box-Plot für die Verteilung der Scores für smear layer in den koronalen Wurzelkanalproben dargestellt.

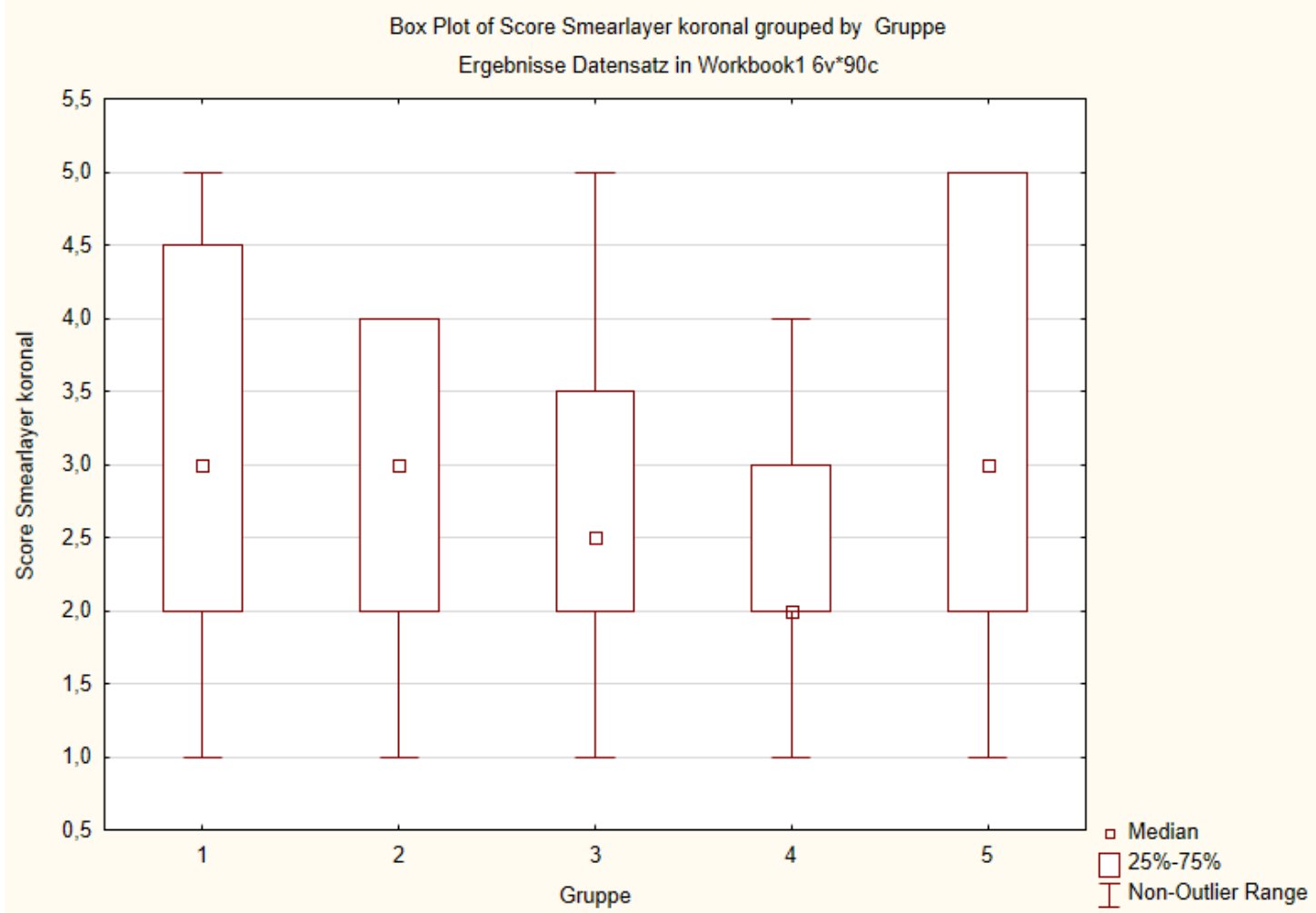

Abbildung 24: Verteilung der Scores für smear layer für den koronalen Wurzelkanalanteil (1 Handspülung, 2 EndoActivator ${ }^{\circledR}, 3$ IrriSafe ${ }^{\mathrm{TM}}$, 4 EDDY $^{\circledR}$, 5 Kontrollgruppe) 
In Abbildung 25 wird die Verteilung der Scores für smear layer für die koronalen Wurzelkanalproben grafisch dargestellt.

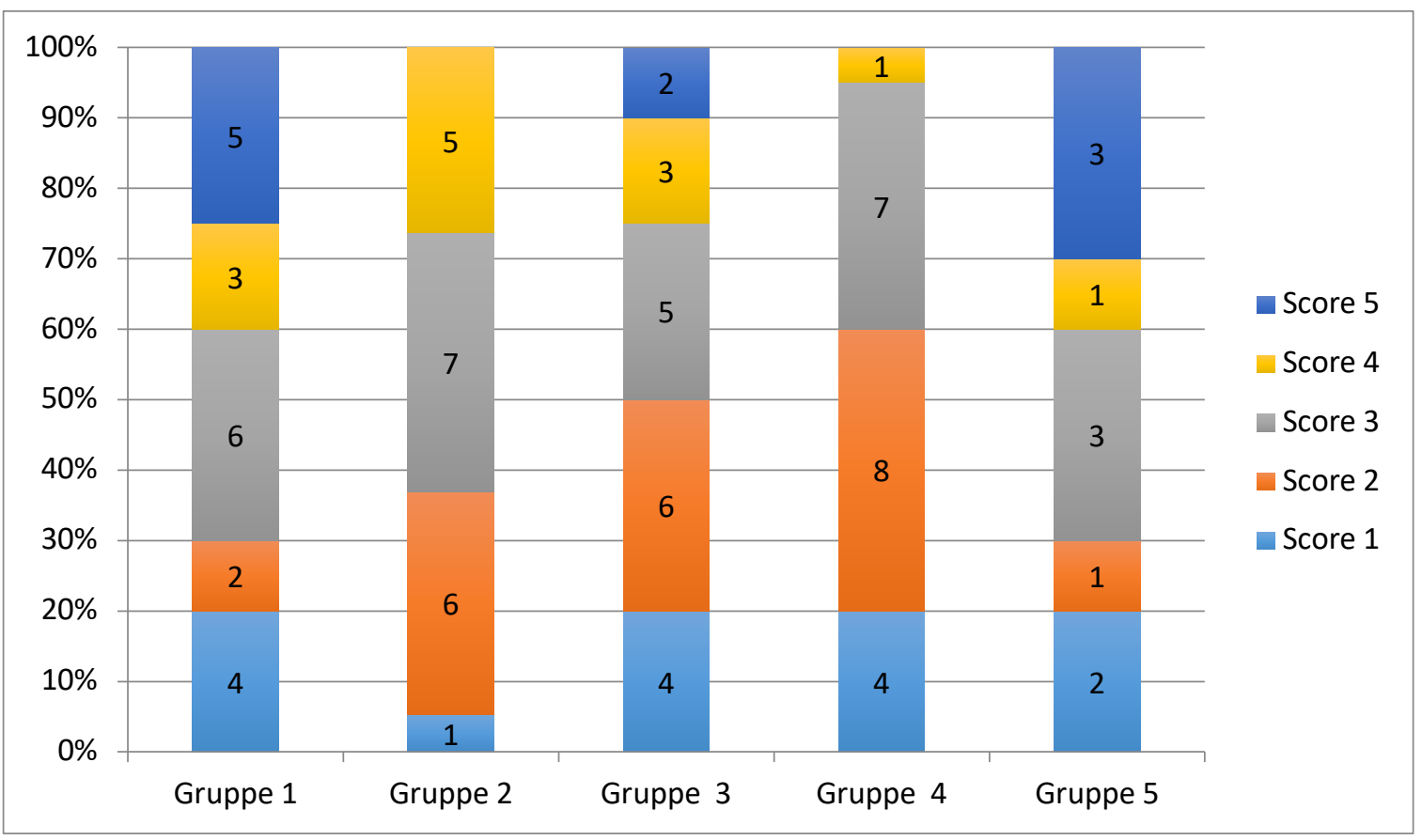

Abbildung 25: Verteilung der Scores für smear layer in den koronalen Wurzelkanalanteilen (1 Handspülung, 2 EndoActivator ${ }^{\circledR}, 3$ IrriSafe ${ }^{\mathrm{TM}}, 4$ EDDY $^{\circledR}, 5$ Kontrollgruppe)

Auch bei der Entfernung des smear layer ist der Vorteil der aktivierten Gruppen gegenüber der dynamischen Handspülung ersichtlich. Bei Betrachtung der Scores 1 und 2 schneidet die dynamische Handspülung am schlechtesten mit einem Ergebnis von 30 \% ab. Es folgen die Gruppen 2 (EndoActivator ${ }^{\circledR}$ ) und 3 (IrriSafe ${ }^{\mathrm{TM}}$ ), die je auf 37,5 bzW. 50 \% kommen. Das beste Ergebnis zeigt hier Gruppe $4\left(\right.$ EDDY $\left.^{\circledR}\right)$ mit einem Ergebnis von 60 \%.

\subsubsection{Entfernung des smear layer im apikalen Wurzelkanalabschnitt}

In Abbildung 26 ist der Box-Plot für die Verteilung der Scores für smear layer in den apikalen Wurzelkanalproben dargestellt. 
Box Plot of Score Smearlayer apical grouped by Gruppe

Ergebnisse Datensatz in Workbook1 $6 \mathrm{v}^{*} 90 \mathrm{c}$

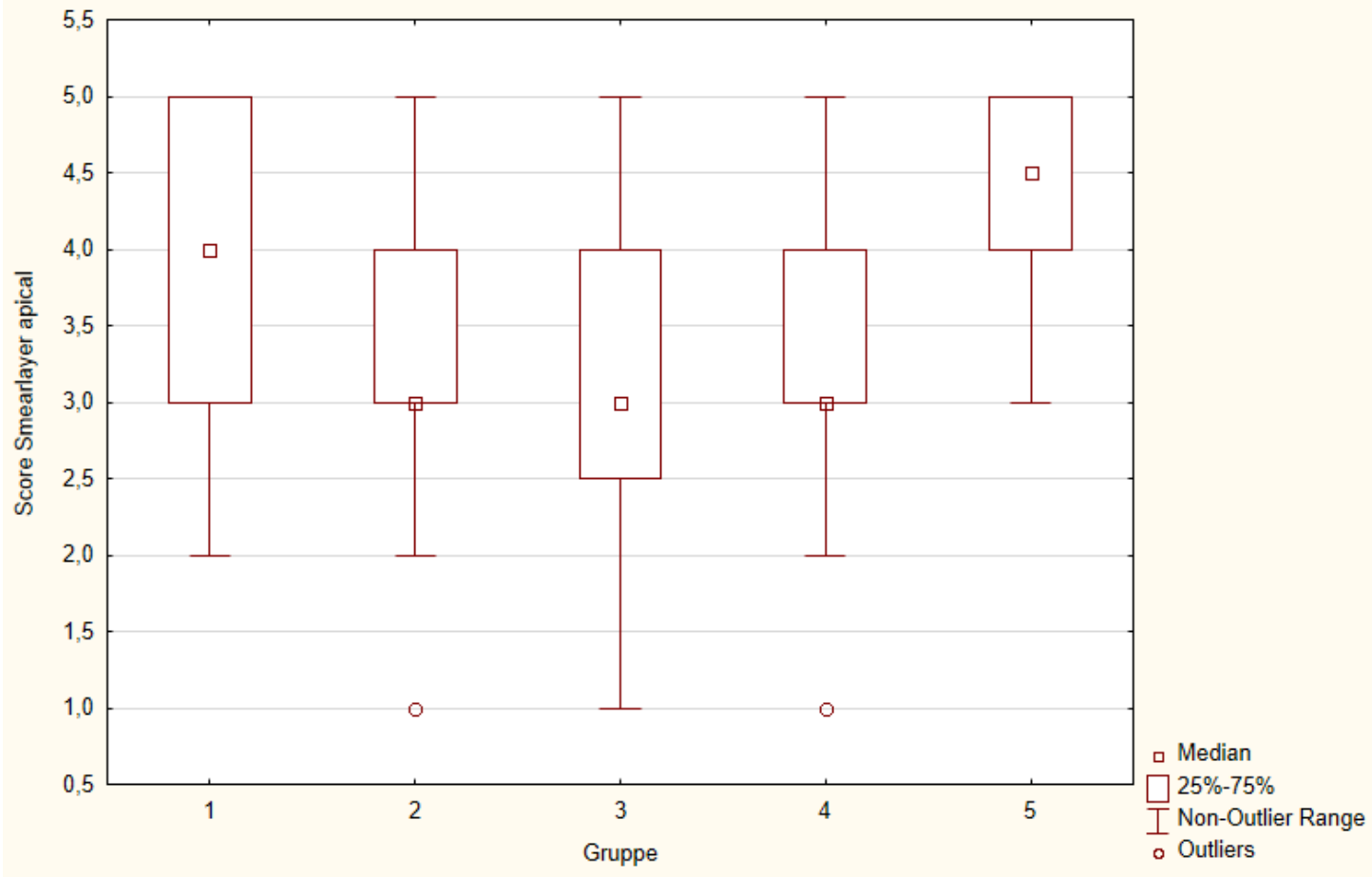

Abbildung 26: Verteilung der Scores für smear layer im apikalen Wurzelkanalanteil. (1 Handspülung, 2 EndoActivator ${ }^{\circledR}, 3$ IrriSafe ${ }^{\mathrm{TM}}$, 4 EDDY ${ }^{\circledR}$, 5 Kontrollgruppe)

Im apikalen Wurzelkanalanteil liegen die schlechtesten Scores vor. Wie in Abbildung 26 ersichtlich, liegen die Mediane der Gruppen insgesamt höher. Den schlechtesten Wert erhält hier die Gruppe 1 (dynamische Handspülung) mit einem Wert von 4. Die Scores 1 und 2 wurden kaum vergeben.

In Abbildung 27 werden die Scores für smear layer für die apikalen Wurzelkanalproben grafisch dargestellt. 


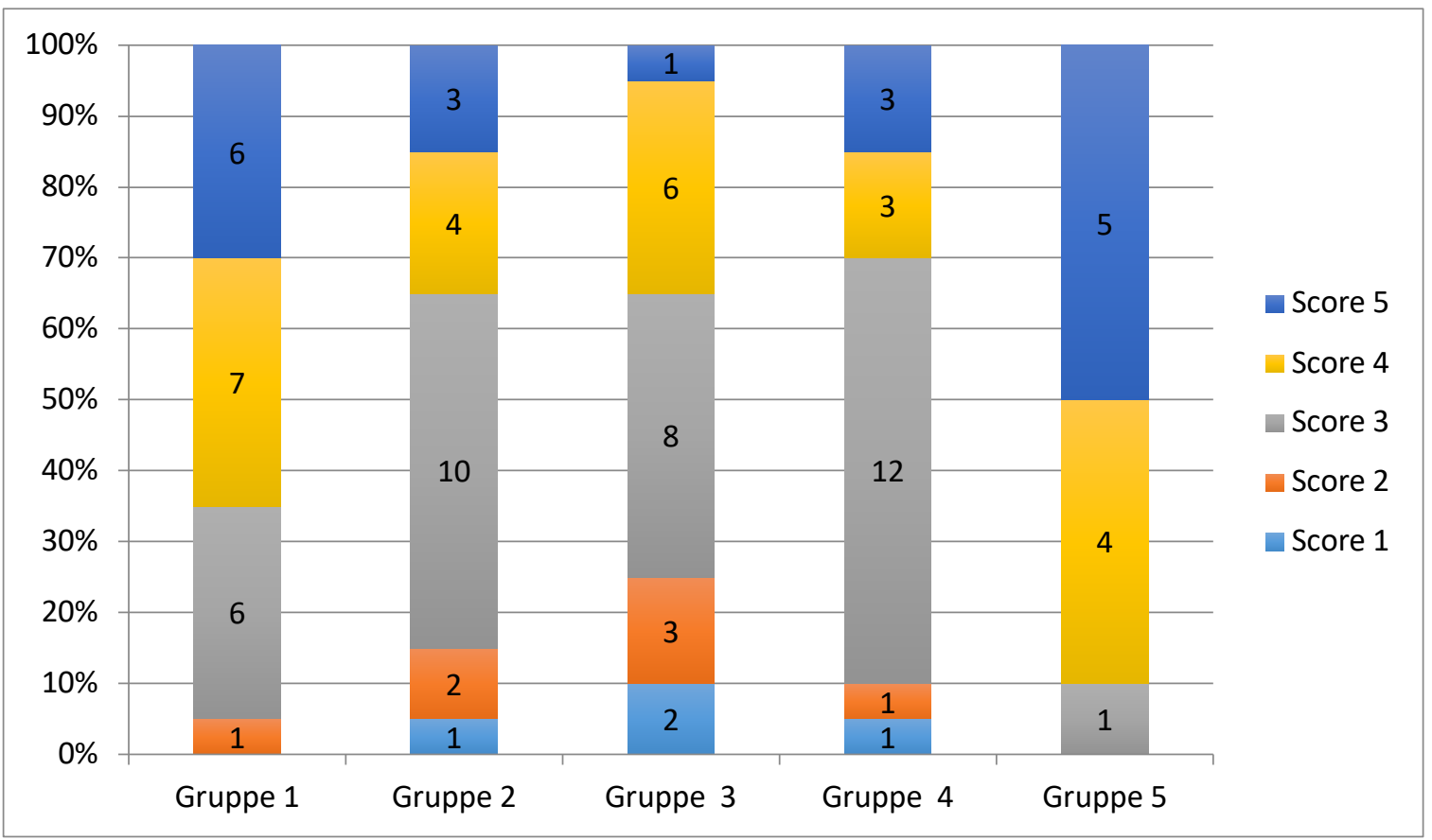

Abbildung 27: Verteilung der Scores für smear layer im apikalen Wurzelkanalanteil (1 Handspülung, 2 EndoActivator ${ }^{\circledR}, 3$ IrriSafe $^{\text {TM }}$, 4 EDDY ${ }^{\circledR}$, 5 Kontrollgruppe)

Die Scores 1 und 2 (Kein smear layer, überwiegend offene Dentintubuli bzw. kleinere Bezirke mit dünnem smear layer, einige offene Dentintubuli) wurden nur selten erreicht. Das beste Ergebnis zeigt dabei Gruppe 3 (IrriSafe ${ }^{\mathrm{TM}}$ ) mit $25 \%$, darauf folgen die schallaktivierten Gruppen 2 und 4 mit jeweils 15 bzw. $10 \%$. Das schlechteste Ergebnis zeigt hierbei Gruppe 1, in der kein Score von 1 vergeben wurde. Ebenso zeigt diese Gruppe mit 30 \% den höchsten Anteil eines Scores von 5.

\subsubsection{Entfernung des smear layer im koronalen und apikalen Kanalanteil}

Beim Vergleich der koronalen und apikalen Reinigungswirkung in Bezug auf den smear layer zeigt sich ein ähnliches Ergebnis wie beim Debris. Die koronalen Proben zeigen deutlich bessere Ergebnisse als die der apikalen Bereiche. So wurde der Score 1 koronal 13 mal vergeben, im apikalen Bereich lediglich 4 mal. Die dynamische Handspülung erreicht diesen Score im apikalen Wurzelkanalabschnitt überhaupt nicht. Beim Score 2 liegt ebenfalls ein deutlicher Unterschied vor. So wurden in den koronalen Proben in 22 
Fällen kleinere Bezirke mit dünnem smear layer und einigen offenen Dentintubuli festgestellt. Bei den apikalen Proben lag dieser Wert bei 7. Ab dem Score 3 verlagert sich das Gleichgewicht hin zu den apikalen Wurzelkanalabschnitten.

\subsection{Gruppe 1: Dynamische Handspülung}

\subsubsection{Debris}

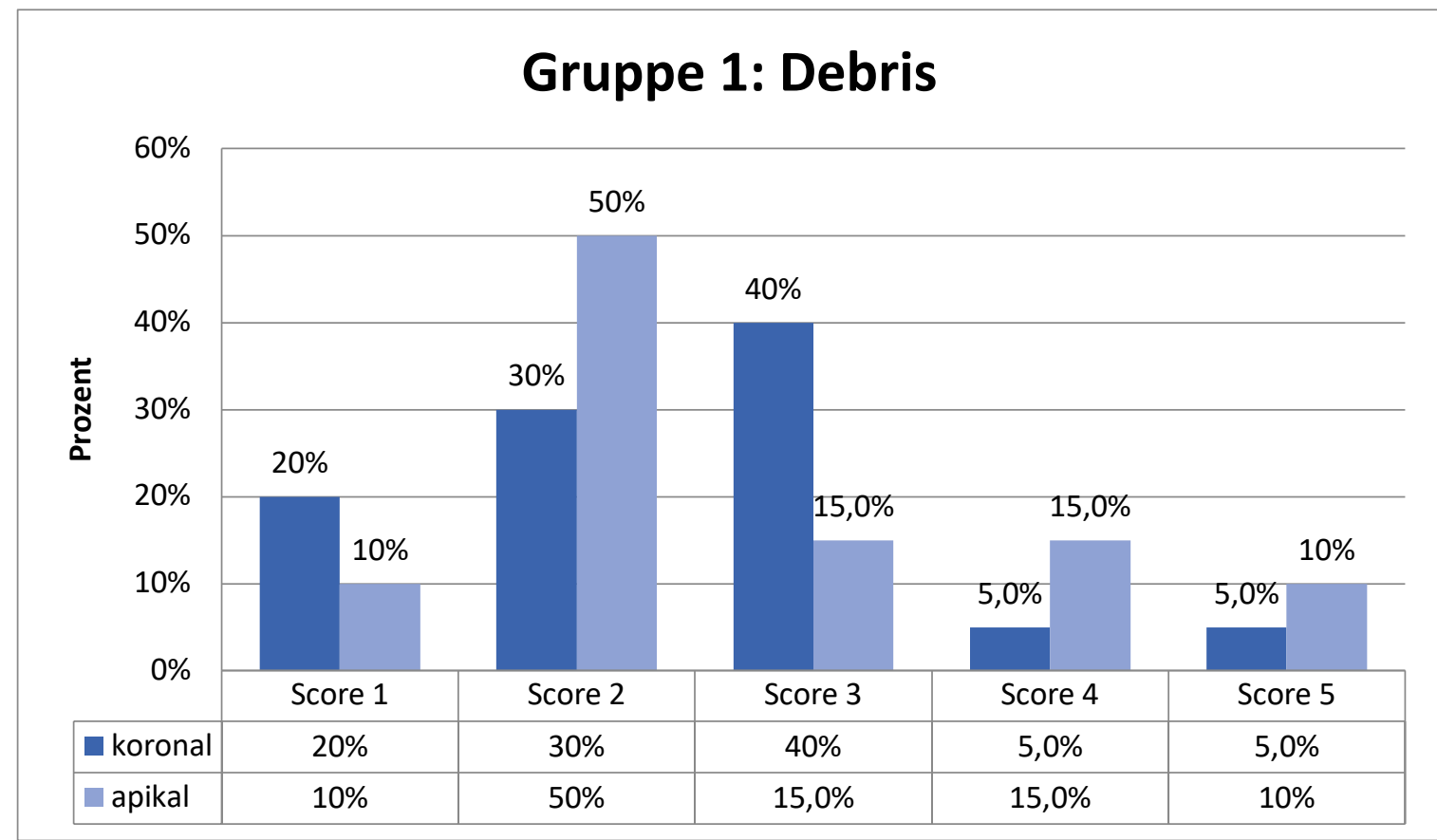

Abbildung 28: Verteilung der Scores für Debris in Gruppe 1 (Dynamische Handspülung)

In Gruppe 1 zeigen sich mit einem Score von 1 oder 250 \% der Wurzelkanalwände im koronalen Bereich sauber. Im apikalen Wurzelkanalabschnitt sind es 60 \%. 40 \% der Kanalwände im koronalen Wurzelkanalabschnitt sind mit zahlreichen Debrisinseln bedeckt, die aber noch weniger als die Hälfte der Oberfläche einnehmen (Score 3). Apikal liegt der Wert mit $15 \%$ niedriger. Auf eine über $50 \%$ bedeckte bzw. vollständig bedeckte Wurzelkanalwand entfallen mit den Scores 4 und $510 \%$ bzw. 25 $\%$. 


\subsubsection{Smear Layer}

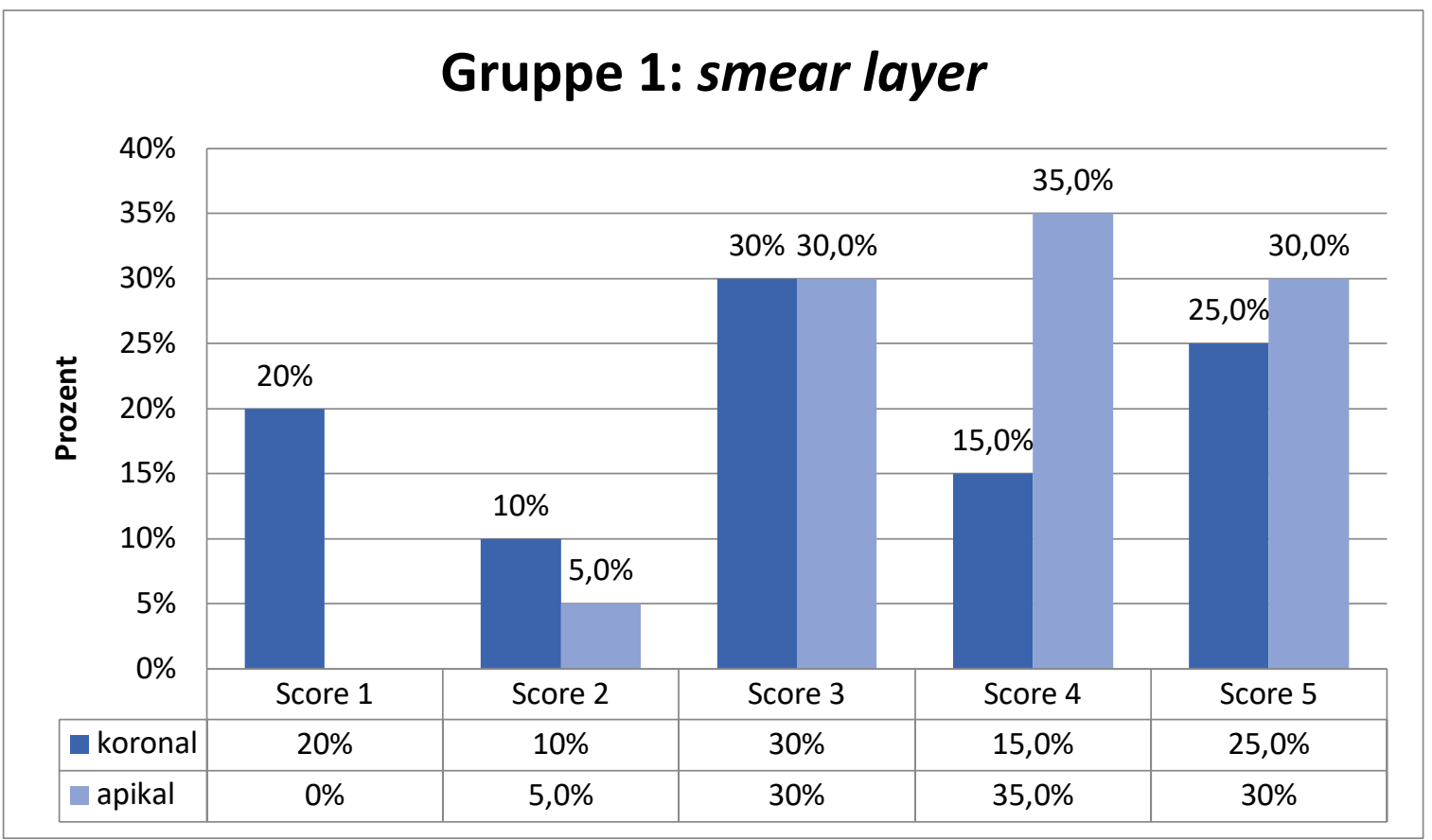

Abbildung 29: Verteilung der Scores für smear layer in Gruppe 1 (Dynamische Handspülung)

Das Ergebnis der smear layer-Beurteilung verschiebt sich deutlich zu den Scores 3 - 5. Während im koronalen Bereich noch $30 \%$ der Wände als sauber zu bewerten sind und keinen smear layer (Score 1) oder nur kleine Bezirke mit dünnem smear layer (Score 2) zeigen, liegt der Wert apikal mit 5 $\%$ sehr niedrig. Bei den restlichen Proben bedeckt die Schmierschicht die gesamte Kanalwand in unterschiedlich starker Ausprägung. 


\subsection{Gruppe 2: EndoActivator®}

\subsubsection{Debris}

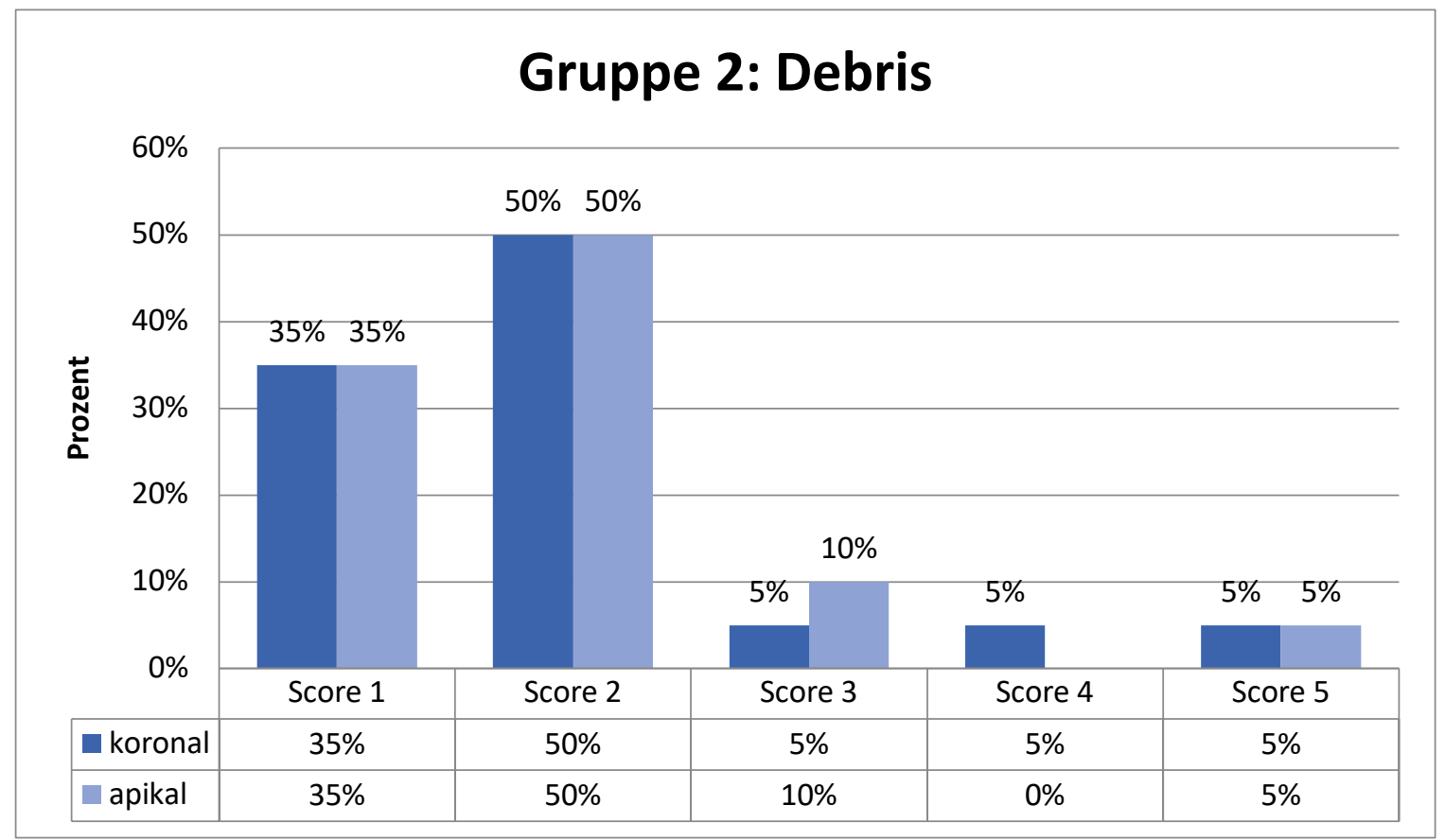

Abbildung 30: Verteilung der Scores für Debris in Gruppe 2 (EndoActivator $\left.{ }^{\circledR}\right)$

In der Gruppe 2 stellen sich $85 \%$ der Wurzelkanalwände in beiden Abschnitten als sauber oder mit kleinen Debrisinseln dar (Score 1 und 2). Somit sind deutlich mehr Wandanteile sauberer als bei der Dynamischen Handspülung. 


\subsubsection{Smear Layer}

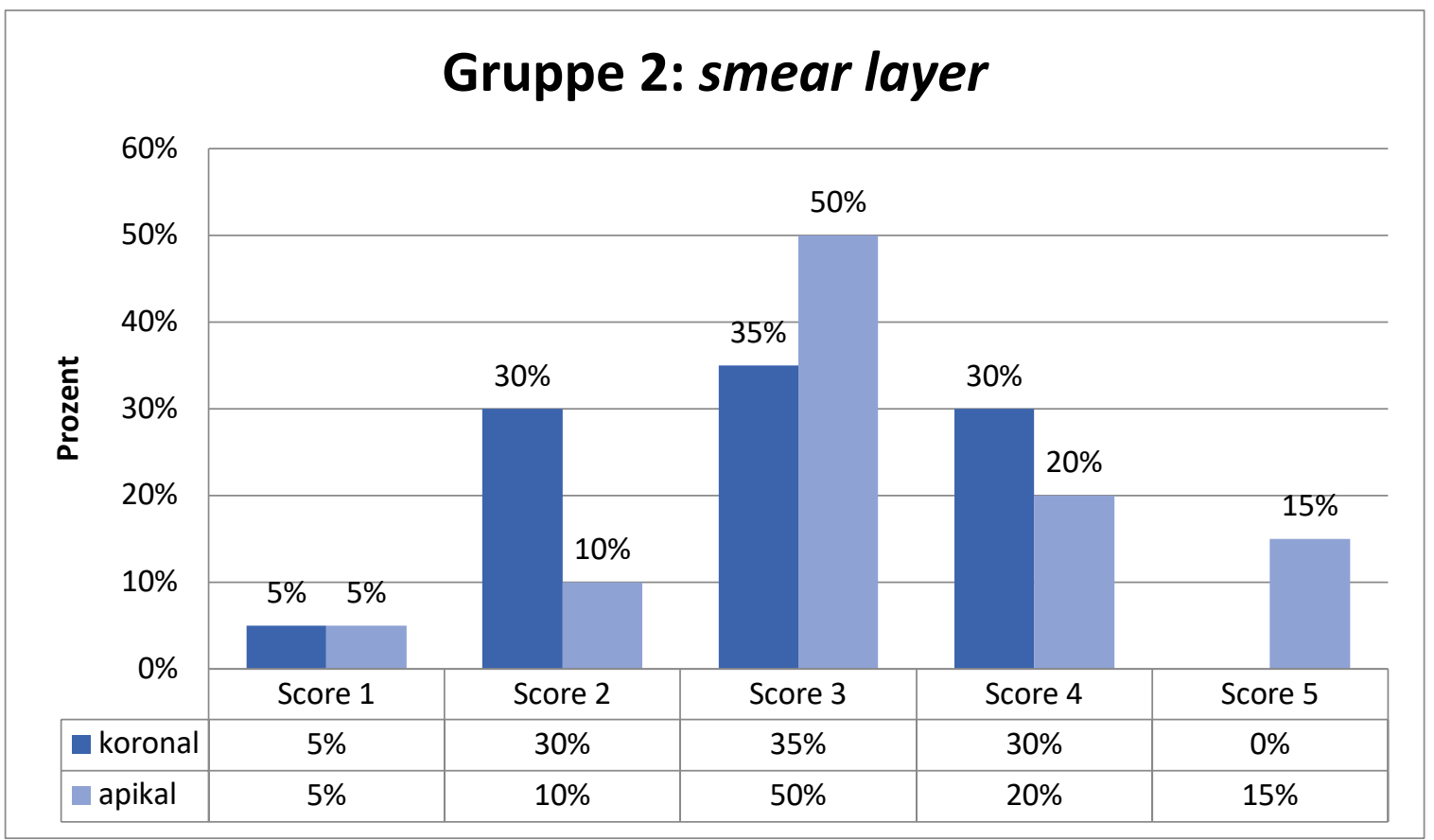

Abbildung 31: Verteilung der Scores für smear layer in Gruppe 2 (EndoActivator®)

Bei der Beurteilung des smear layer zeigen sich überwiegend nicht saubere Wände. So entfällt mit $35 \%-50 \%$ ein Großteil der vergebenen Scores auf den Wert 3 (homogener smear layer, der die gesamte Kanalwand bedeckt). Zieht man die Scores 4 und 5 hinzu, so liegen in dieser Gruppe in 65 \% (koronal) bzw. 70 \% (apikal) der Fälle nicht saubere Wurzelkanalwände vor. Lediglich bei 25 \% der Proben wurden die Kanalwände als sauber bewertet, davon zeigt sich der überwiegende Teil im koronalen Wurzelkanalbereich.

Im Vergleich zur Gruppe 1 zeigen sich beim EndoActivator $®$ bessere Ergebnisse in Bezug auf Debris- und smear layer-Entfernung. 


\subsection{Gruppe 3: IrriSafe ${ }^{\mathrm{TM}}$}

\subsubsection{Debris}

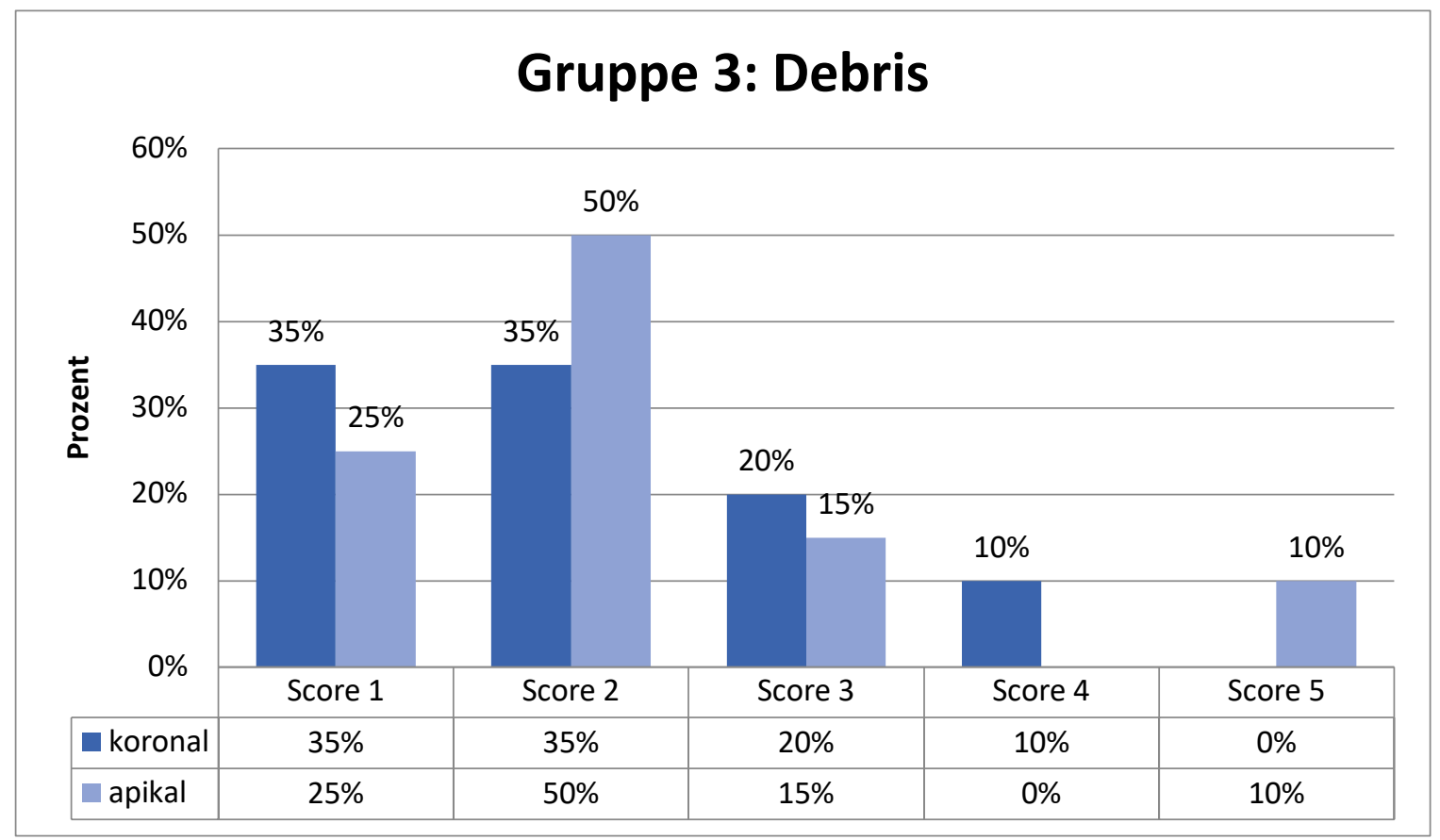

Abbildung 32: Verteilung der Scores für Debris in Gruppe 3 (IrriSafe ${ }^{\mathrm{TM}}$ )

In der Gruppe der passiven Ultraschallspülung (IrriSafe ${ }^{\mathrm{TM}}$ ) liegen in 72,5 \% der gesamten Proben (Score 1 und 2) überwiegend saubere Wurzelkanalwände vor. Im Vergleich mit dem EndoActivator® (Gruppe 2) zeigen sich bei der PUI häufiger die Scores $3-5$. Hier beträgt der Anteil an nicht sauberen Wänden 27,5 \% gesamt, in der Gruppe 2 liegt dieser Prozentsatz bei 12,5 \% gesamt. Auch in dieser Gruppe lässt sich ein Vorteil gegenüber der dynamischen Handspülung erkennen. 


\subsubsection{Smear Layer}

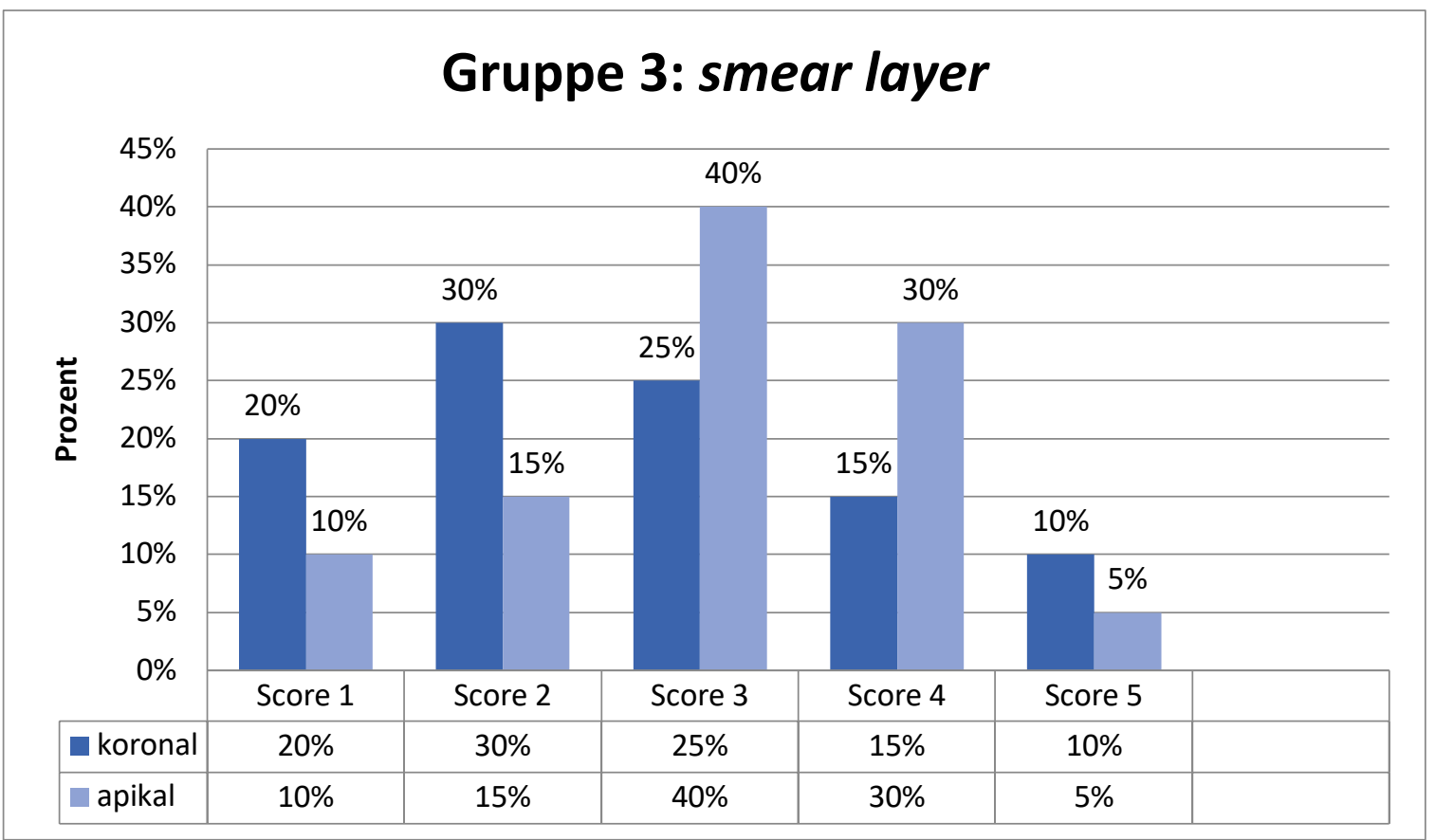

Abbildung 33: Verteilung der Scores für smear layer in Gruppe 3 (IrriSafe ${ }^{\mathrm{TM}}$ )

Ähnlich wie in Gruppe 2 findet auch hier im Vergleich zum Debris eine Verschiebung in den mittleren Score-Bereich statt. 37,5\% der gesamten Proben zeigen sich sauber, dies sind 12,5\% mehr als in der Gruppe 2 (EndoActivator®). Die Häufigkeit der Scores 4 und 5 deckt sich weitgehend mit der Verteilung in Gruppe 2, der Score 5 kommt bei der PUI dabei auch im koronalen Bereich vor. Wiederum ist ein Vorteil der aktivierten Gruppe 3 gegenüber der dynamischen Handspülung zu beobachten. 


\subsection{Gruppe 4: EDDY ${ }^{\circledR}$}

\subsubsection{Debris}

\section{Gruppe 4: Debris}

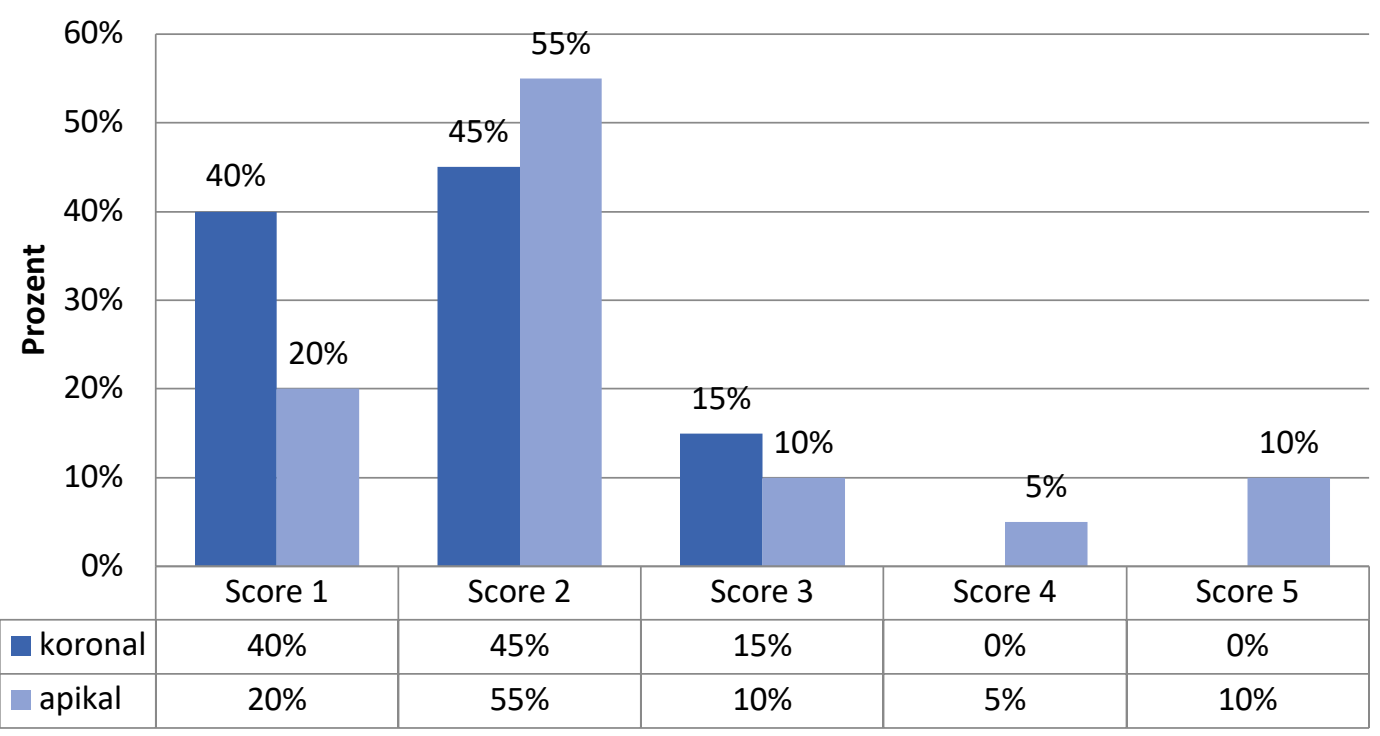

Abbildung 34: Verteilung der Scores für Debris in Gruppe 4 (EDDY $\left.{ }^{\circledR}\right)$

Gruppe 4 zeigt in 80 \% der Proben saubere Kanalwände mit einem Score von 1 oder 2. Damit sind die Ergebnisse bei der Debris Entfernung sehr ähnlich denen der anderen schallaktivierten Gruppe 2 (EndoActivator®). Wie bei allen aktivierten Gruppen zeigt sich erneut ein Vorteil gegenüber der dynamischen Handspülung. Die Scores 3-5 wurden vorwiegend im apikalen Wurzelkanalbereich vergeben. 


\subsubsection{Smear Layer}

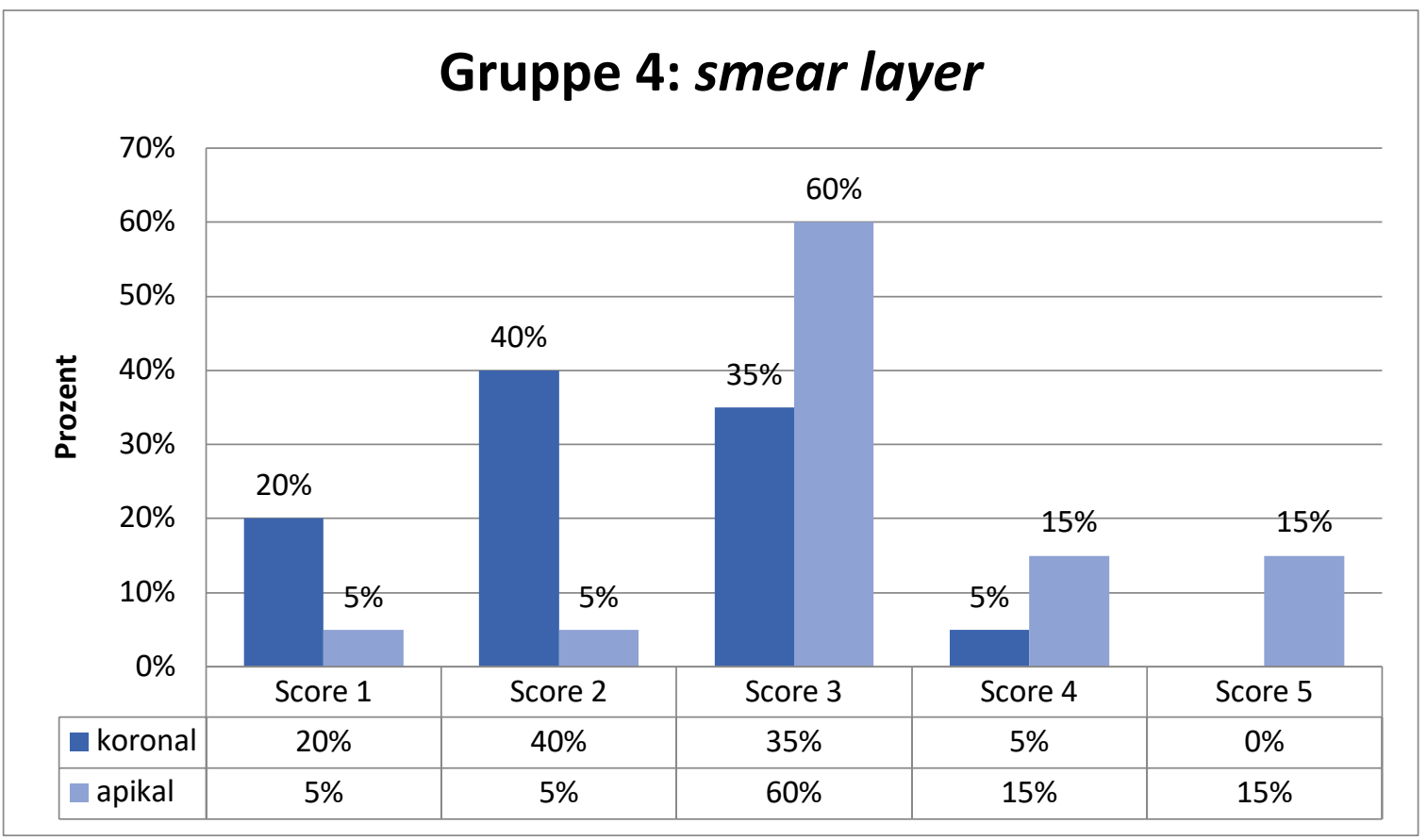

Abbildung 35: Verteilung der Scores für smear layer in Gruppe 4 (EDDY ${ }^{\circledR}$ )

Saubere Wurzelkanalwände (Score 1 und 2) liegen in Gruppe 4 in 35 \% der Proben vor. Dieses Ergebnis ist sehr ähnlich der Gruppe 3 (PUI), wo das Ergebnis $37,5 \%$ beträgt. Auffallend in Gruppe 4 ist, dass die sauberen Wände fast ausschließlich koronal vorliegen. Der Score 3 wurde mit 47,5\% in dieser Gruppe am häufigsten vergeben, wovon $30 \%$ auf den apikalen Wurzelkanalanteil entfallen. 


\subsection{Gruppe 5: Kontrollgruppe}

\subsubsection{Debris}

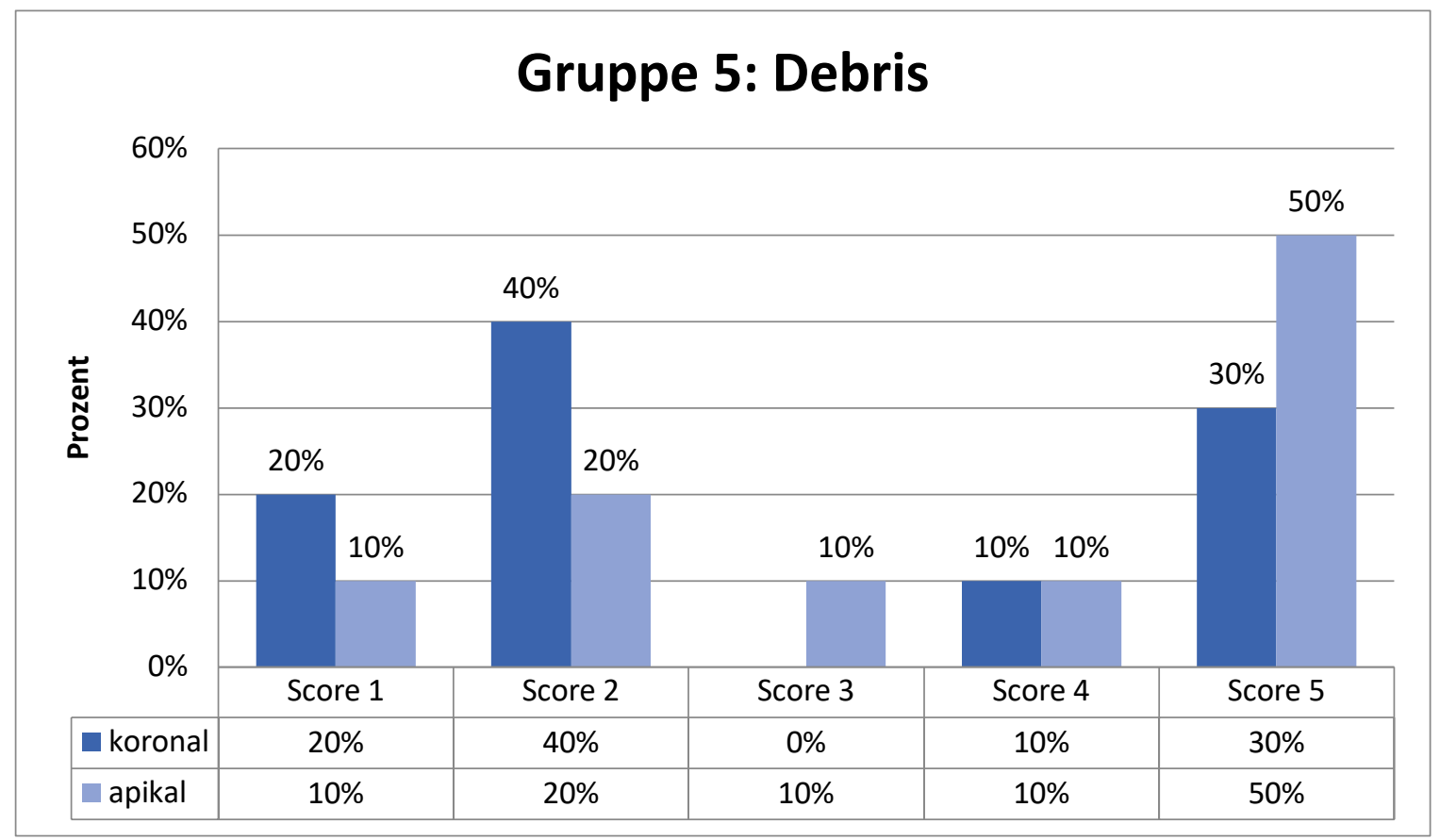

Abbildung 36: Darstellung der Scores für Debris in Gruppe 5 (Kontrollgruppe)

Die Kontrollgruppe zeigt in Bezug auf die Entfernung des Debris im apikalen Bereich zu 60 \% Wurzelkanalwände mit einem Score von 4 oder 5. Dies bedeutet, dass die Wurzelkanalwände zu einem kleineren Anteil eine Oberfläche mit zahlreichen Debrisinseln aufweisen, die aber bereits über $50 \%$ der Oberfläche einnehmen. Der weit größere Anteil zeigt dabei eine durchgehende Debrisschicht auf der Wurzelkanaloberfläche. Koronal fällt das Ergebnis mit $60 \%$ sauberen Oberflächen deutlich positiver aus. 


\subsubsection{Smear Layer}

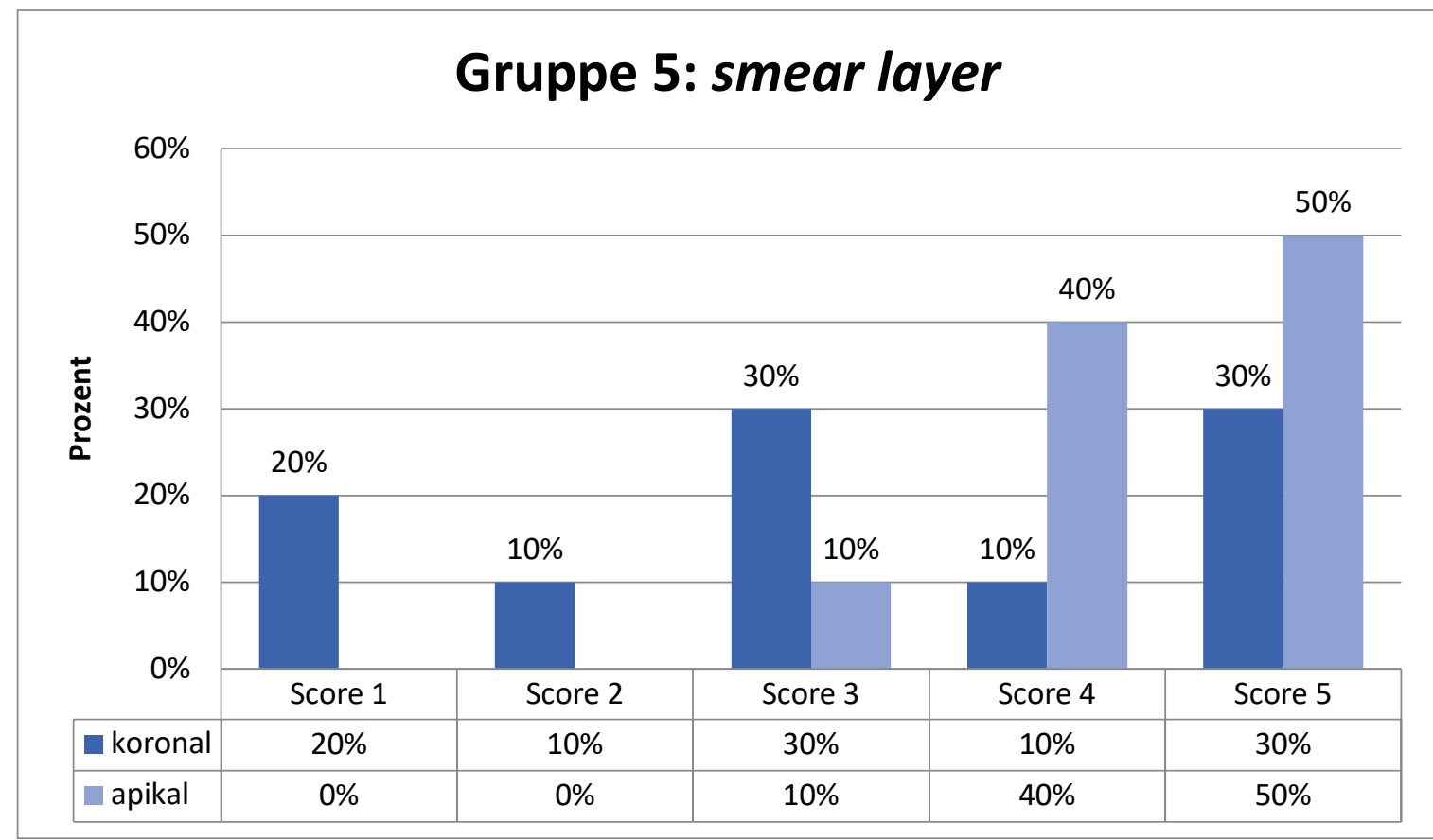

Abbildung 37: Darstellung der Scores für smear layer in Gruppe 5 (Kontrollgruppe)

Bei der Entfernung des smear layer im apikalen Bereich zeigt sich deutlich, dass ohne Spülung keine Reinigungswirkung zu erreichen ist. $90 \%$ der Proben haben hier einen Score von 4 oder 5, was einem dichtem smear layer auf der gesamten Kanalwand entspricht. Koronal verteilen sich die scores deutlich gleichmäßiger, wobei auch hier noch $40 \%$ auf die Scores 4 und 5 entfallen.

\subsection{Statistische Auswertung}

Die statistische Auswertung erfolgte mit dem Programm Statistica (StatSoft Europe $\mathrm{GmbH}$, Hamburg). Die Grafiken wurden ebenfalls mit Statistica sowie mit Excel und Word für Mac (Microsoft Corporation, Redmond, Washington, USA) erstellt.

Die Ergebnisse der Versuchsgruppen wurden global mit dem Kruskal-WallisTest verglichen. Der Signifikanzwert ( $p$-Wert) wurde auf $p=0,05$ festgelegt. Ergab die Auswertung einen signifikanten Unterschied zwischen den Gruppen, so wurde im Anschluss ein Post-Hoc-Test durchgeführt. Dabei 
wurde mit Hilfe des Mann-Whitney-U-Tests Gruppe gegen Gruppe verglichen um festzulegen, zwischen welchen Gruppen der signifikante Unterschied bestand. War der Unterschied als signifikant einzustufen, so wurde die Nullhypothese (Kein Unterschied zwischen den Gruppen) abgelehnt und die Alternativhypothese (Überlegene Reinigungswirkung der jeweiligen Aktivierungsmethode) konnte angenommen werden.

\subsubsection{Ergebnisse}

Ein Überblick über alle für die statistische Auswertung relevanten Ergebnisse findet sich im Anhang in den Tabellen 12-36.

\subsubsection{Auswertung}

Zunächst wurden die Versuchsgruppen global auf signifikante Unterschiede in Bezug auf die Scores für Debris und smear layer überprüft. Für diese Überprüfung wurde der Kruskal-Wallis-Test angewandt und für die vier Variablen Debris koronal, Debris apikal, smear layer koronal und smear layer apikal durchgeführt.

\subsubsection{Auswertung Debris koronal}

\begin{tabular}{|l|l|l|l|l|}
\hline \multicolumn{5}{|l|}{ Kruskal-Wallis Test: $\mathrm{H}(4, \mathrm{~N}=89)=8,385983 \mathbf{p}=\mathbf{0 , 0 7 8 4}$} \\
\hline Gruppe & Code & Valid N & Sum of Ranks & Mean Rank \\
\hline 1 & 1 & 20 & 1087,500 & 54,37500 \\
\hline 2 & 2 & 19 & 722,000 & 38,00000 \\
\hline 3 & 3 & 20 & 883,000 & 44,15000 \\
\hline 4 & 4 & 20 & 752,000 & 37,60000 \\
\hline 5 & 5 & 10 & 560,000 & 56,05000 \\
\hline
\end{tabular}

Tabelle 4: Ergebnisse des Kruskal-Wallis-Tests für Debris koronal (Statistica)

In Bezug auf die koronale Debris-Entfernung ließ sich kein signifikanter Unterschied zwischen den getesteten Aktivierungsmethoden feststellen. Der p-Wert liegt mit 0,0784 oberhalb des Signifikanzniveaus $\alpha$. Die Nullhypothese 
(Kein Unterschied zwischen den Aktivierungsmethoden) wurde somit beibehalten.

5.8.2.2 Auswertung Debris apikal

\begin{tabular}{|l|l|l|l|l|}
\hline \multicolumn{5}{|l|}{ Kruskal-Wallis Test: $\mathrm{H}(4, \mathrm{~N}=90)=12,42023 \mathrm{p}=\mathbf{0 , 0 1 4 5}$} \\
\hline Gruppe & Code & Valid N & Sum of Ranks & Mean Rank \\
\hline 1 & 1 & 20 & 1031,000 & 51,55000 \\
\hline 2 & 2 & 20 & 700,500 & 35,02500 \\
\hline 3 & 3 & 20 & 833,000 & 41,65000 \\
\hline 4 & 4 & 20 & 872,000 & 43,60000 \\
\hline 5 & 5 & 10 & 658,000 & 65,85000 \\
\hline
\end{tabular}

Tabelle 5: Ergebnisse des Kruskal-Wallis-Tests für Debris apikal (Statistica)

Der globale Vergleich der Gruppen für Debris apikal zeigt einen p-Wert von 0,0145. Dieser liegt unterhalb des Signifikanzniveaus und somit kann ein signifikanter Unterschied angenommen werden.

Mit Hilfe des Mann-Whitney-U-Tests erfolgte der Vergleich von Gruppe gegen Gruppe. Die Ergebnisse sind im Folgenden dargestellt.

In Tabelle 6 sind die p-Werte der Einzeltests für Debris apikal zusammengefasst. Eine ausführliche Übersicht über die einzelnen Tests findet sich im Anhang (8.2.2.) in den Tabellen 17-26. 


\begin{tabular}{|c|c|c|c|c|c|}
\hline & $\begin{array}{l}\text { Dynamische } \\
\text { Handspülung }\end{array}$ & $\begin{array}{c}\text { Endo- } \\
\text { Activator }^{\circledR}\end{array}$ & IrriSafe ${ }^{T M}$ & EDDY $^{\circledR}$ & $\begin{array}{l}\text { Kontroll - } \\
\text { gruppe }\end{array}$ \\
\hline $\begin{array}{l}\text { Dynamische } \\
\text { Handspülung }\end{array}$ & & $p=0,038516$ & $p=0,213389$ & $p=0,316898$ & $p=0,082253$ \\
\hline $\begin{array}{l}\text { Endo } \\
\text { Activator }{ }^{\circledR}\end{array}$ & & & $p=0,417078$ & $p=0,285306$ & $p=0,006818$ \\
\hline IrriSafe ${ }^{T M}$ & & & & $p=0,818150$ & $p=0,023471$ \\
\hline $\mathrm{EDDY}^{\circledR}$ & & & & & $p=0,031107$ \\
\hline Kontrollgruppe & & & & & \\
\hline
\end{tabular}

Tabelle 6: Zusammenfassung der Ergebnisse der Mann-Whitney-U-Tests für Debris apikal

Die hier dargestellten p-Werte wurden im Anschluss der Bonferroni-Korrektur unterzogen, um die alpha-Fehler-Kumulierung zu neutralisieren, da es sich bei dem verwendeten Testverfahren um ein multiples Testen innerhalb derselben Stichproben handelte.

In Tabelle 7 sind die nach der Bonferroni-Methode korrigierten p-Werte für die Variable „Debris apikal“ zusammengefasst.

\begin{tabular}{|c|c|c|c|c|c|}
\hline & $\begin{array}{l}\text { Dynamische } \\
\text { Handspülung }\end{array}$ & $\begin{array}{c}\text { Endo- } \\
\text { Activator }\end{array}$ & IrriSafe ${ }^{T M}$ & EDDY $^{\circledR}$ & $\begin{array}{l}\text { Kontroll- } \\
\text { gruppe }\end{array}$ \\
\hline $\begin{array}{l}\text { Dynamische } \\
\text { Handspülung }\end{array}$ & & $p=0,38516$ & $p=2,13389$ & $p=3,16898$ & $p=0,82253$ \\
\hline $\begin{array}{l}\text { Endo- } \\
\text { Activator }{ }^{\circledR}\end{array}$ & & & $p=4,17078$ & $p=2,85306$ & $p=0,06818$ \\
\hline IrriSafe ${ }^{\mathrm{TM}}$ & & & & $p=8,18150$ & $p=0,23471$ \\
\hline EDDY $^{\circledR}$ & & & & & $p=0,31107$ \\
\hline Kontroll-gruppe & & & & & \\
\hline
\end{tabular}

Tabelle 7: Zusammenfassung der nach Bonferroni korrigierten p-Werte für Debris apikal

In den Einzelvergleichen zwischen den Spülmethoden konnten keine signifikanten Unterschiede in Bezug auf die Verteilung der Scores für die 
apikale Debrisentfernung festgestellt werden. So liegen alle $p$-Werte oberhalb des Signifikanzniveaus von $\alpha=0,05$. Aufgrund des gewählten Testverfahrens mussten die p-Werte mit Hilfe der Bonferroni-Methode korrigiert werden. Die Annahme eines signifikanten Unterschiedes durch den Kruskal-Wallis-Test bestätigt sich für die untersuchten Gruppen nicht. Die Nullhypothese wird weiterhin angenommen.

5.8.2.3 Auswertung smear layer koronal

\begin{tabular}{|l|l|l|l|l|}
\hline \multicolumn{5}{|l|}{ Kruskal-Wallis Test: $H(4, N=89)=6,545712 p=0,1619$} \\
\hline Gruppe & Code & Valid N & Sum of Ranks & Mean Rank \\
\hline 1 & 1 & 20 & 1042,500 & 52,12500 \\
\hline 2 & 2 & 20 & 902,500 & 47,50000 \\
\hline 3 & 3 & 20 & 844,500 & 42,22500 \\
\hline 4 & 4 & 20 & 688,500 & 34,42500 \\
\hline 5 & 5 & 10 & 527,500 & 52,70000 \\
\hline
\end{tabular}

Tabelle 8: Ergebnisse des Kruskal-Wallis-Tests für smear layer koronal (Statistica)

Der Kruskal-Wallis-Test zeigt bei der Entfernung von smear layer im koronalen Bereich keinen signifikanten Unterschied zwischen den getesteten Aktivierungsmethoden. Der p-Wert liegt mit 0,1619 oberhalb des Signifikanzniveaus $\alpha$. Die Nullhypothese (Kein Unterschied zwischen den Aktivierungsmethoden) wird weiter angenommen. 
5.8.2.4 Auswertung smear layer apikal

\begin{tabular}{|l|l|l|l|l|}
\hline \multicolumn{5}{|l|}{ Kruskal-Wallis Test: $\mathrm{H}(4, \mathrm{~N}=90)=16,19238 \mathbf{p}=\mathbf{0 , 0 0 2 8}$} \\
\hline Gruppe & Code & Valid N & Sum of Ranks & Mean Rank \\
\hline 1 & 1 & 20 & 1100,500 & 55,02500 \\
\hline 2 & 2 & 20 & 805,000 & 40,25000 \\
\hline 3 & 3 & 20 & 713,500 & 35,67500 \\
\hline 4 & 4 & 20 & 796,500 & 39,82500 \\
\hline 5 & 5 & 10 & 679,500 & 67,95000 \\
\hline
\end{tabular}

Tabelle 9: Ergebnisse des Kruskal-Wallis-Tests für smear layer apikal (Statistica)

Die Auswertung für die Variable „smear layer apikal“ zeigt erneut einen pWert der unterhalb des Signifikanzniveaus a liegt. Somit kann ein signifikanter Unterschied zwischen den Gruppen angenommen werden. 
In Tabelle 10 sind die p-Werte der Einzeltests für smear layer apikal zusammengefasst. Eine ausführliche Übersicht über die einzelnen Tests findet sich im Anhang (8.2.2.) in den Tabellen 27-36.

\begin{tabular}{|c|c|c|c|c|c|}
\hline & $\begin{array}{l}\text { Dynamische } \\
\text { Handspülung }\end{array}$ & $\begin{array}{c}\text { Endo- } \\
\text { Activator }{ }^{\circ}\end{array}$ & IrriSafe ${ }^{\mathrm{TM}}$ & $\mathrm{EDDY}^{\circledR}$ & $\begin{array}{l}\text { Kontroll- } \\
\text { Gruppe }\end{array}$ \\
\hline $\begin{array}{l}\text { Dynamische } \\
\text { Handspülung }\end{array}$ & & $p=0,076432$ & $p=0,020003$ & $p=0,063893$ & $p=0,179655$ \\
\hline $\begin{array}{l}\text { Endo- } \\
\text { Activator } \AA\end{array}$ & & & $p=0,588507$ & $p=0,989209$ & $p=0,008300$ \\
\hline IrriSafe ${ }^{\mathrm{TM}}$ & & & & $p=0,616775$ & $p=0,002073$ \\
\hline $\mathrm{EDDY}^{\circledR}$ & & & & & $p=0,006379$ \\
\hline $\begin{array}{l}\text { Kontroll- } \\
\text { gruppe }\end{array}$ & & & & & \\
\hline
\end{tabular}

Tabelle 10: Zusammenfassung der Ergebnisse der Mann-Whitney-U-Tests für smear layer apikal

Auch hier erfolgte die Korrektur der $p$-Werte nach Bonferroni. Die so korrigierten Werte sind in Tabelle 36 zusammengefasst.

\begin{tabular}{|c|c|c|c|c|c|}
\hline & $\begin{array}{l}\text { Dynamische } \\
\text { Handspülung }\end{array}$ & $\begin{array}{c}\text { Endo- } \\
\text { Activator }\end{array}$ & IrriSafe ${ }^{\mathrm{TM}}$ & EDDY $^{\circledR}$ & $\begin{array}{l}\text { Kontroll- } \\
\text { gruppe }\end{array}$ \\
\hline $\begin{array}{l}\text { Dynamische } \\
\text { Handspülung }\end{array}$ & & $p=0,76432$ & $p=0,20003$ & $p=0,63893$ & $p=1,79655$ \\
\hline $\begin{array}{l}\text { Endo- } \\
\text { Activator }\end{array}$ & & & $p=5,88507$ & $p=9,89209$ & $p=0,08300$ \\
\hline IrriSafe ${ }^{\mathrm{TM}}$ & & & & $p=6,16775$ & $p=0,02073$ \\
\hline EDDY $^{\circledR}$ & & & & & $p=0,06379$ \\
\hline $\begin{array}{l}\text { Kontroll- } \\
\text { gruppe }\end{array}$ & & & & & \\
\hline
\end{tabular}

Tabelle 11: Nach Bonferroni korrigierte $p$-Werte für die Variable smear layer apikal 
Der Vergleich der aktivierten Gruppen in Bezug auf die Reinigungseffektivität für smear layer im apikalen Bereich zeigt zwischen diesen Gruppen keine signifikanten Unterschiede. Auch hier liegen alle $p$-Werte oberhalb des Signifikanzniveaus $\alpha$. Der durch den Kruskall-Wallis-Test angenommene signifikante Unterschied bestätigt sich auch für die Variable smear layer apikal nicht.

\subsubsection{Zusammenfassung der Auswertung}

Die statistische Auswertung erfolgte zunächst global mit Hilfe des KruskalWallis-Tests, um signifikante Unterschiede zwischen den Gruppen festzustellen. Dieser Test wurde für jede der vier Variablen (1. Debris koronal, 2. Debris apikal, 3. smear layer koronal und 4. smear layer apikal) einzeln durchgeführt. Für die Reinigungseffektivität im koronalen Bereich ergaben die Tests keine signifikanten Unterschiede zwischen den Versuchsgruppen (Variable 1 und 3). Die Untersuchung der Variablen 2 und 4 zeigte, dass statistisch signifikante Unterschiede im apikalen Wurzelkanalbereich anzunehmen waren. Nach Durchführung der Post-Hoc-Tests (Mann-WhitneyU-Test) musste diese Annahme für die getesteten Gruppen verworfen werden, da zwischen den aktivierten Methoden keine signifikanten Unterschiede gefunden werden konnten. Es ist dabei anzunehmen, dass die signifikanten Unterschiede dabei einen Vergleich mit der Kontrollgruppe betrafen oder aber die Anwendung der Bonferroni-Korrektur nicht berücksichtigten. Dies gilt sowohl für die Variable 2 (Debris apikal) als auch für die Variable 4 (smear layer apikal).

Zusammenfassend kann festgestellt werden, dass die unterschiedlichen Aktivierungsmethoden zwar prozentual leichte Vorteile in bestimmten Bereichen gegenüber anderen Spülmethoden in Bezug auf die Reinigungseffektivität aufweisen, diese sich aber statistisch nicht belegen lassen. Somit ist keine der in dieser Studie verwendeten Aktivierungsmethoden in Hinblick auf Debris- oder smear layer - Entfernung den anderen Methoden überlegen. Auch die dynamische Handspülung zeigt sich als geeignete Methode zur Spülung des Wurzelkanalsystems. 


\section{Diskussion}

Das Ziel der vorliegenden Studie war der Vergleich der Reinigungseffektivität unterschiedlicher aktivierter Wurzelkanalspültechniken. Der Vergleich erfolgte zwischen der konventionellen dynamischen Handspülung, der schallaktivierten Spülung mit dem EndoActivator ${ }^{\circledR}$ und dem ebenfalls schallaktivierten EDDY ${ }^{\circledR}$-System sowie der ultraschallbasierten Spülung mit IrriSafe $^{\mathrm{TM}}$. Die Wurzelkanäle wurden nach der Präparation nach vorgegebenen standardisierten Spülprotokollen gespült und die Spüllösung entsprechend aktiviert. In der Studie wurden weitestgehend standardisierte Arbeitsabläufe angewendet, die über den gesamten Studienverlauf eingehalten wurden. Die Einhaltung dieser standardisierten Arbeitsabläufe und Bedingungen erlaubt eine objektive Beurteilung der Ergebnisse sowie eine Vergleichbarkeit mit anderen Studien.

\subsection{Diskussion von Material und Methoden}

Als Untersuchungsmaterial dienten 90 extrahierte humane Unterkiefermolaren. Dabei kamen sowohl erste als auch zweite untere Molaren zur Anwendung, die sich in ihrer Wurzelkanalanatomie sehr ähneln. Untersucht wurde jeweils der mesiobukkale Wurzelkanal jedes Zahnes. Aufgrund seiner Eigenschaften wie Querschnittsform, Länge und Krümmungswinkel bietet er gute Voraussetzungen zum Vergleich verschiedener Spülsysteme, da er keine zu einfachen Anforderungen an die Systeme stellt. Auch in vergleichbaren Studien dienten untere Molaren als Untersuchungsmaterial (Al-Ali et al. 2012; Caron et al. 2010; Duque et al. 2016; Elnaghy et al. 2016; Hülsmann et al. 2003; Klyn et al. 2010; Munoz et al. 2012; Rödig et al. 2010; Yeung et al. 2014). Um die Eigenschaften der verschiedenen Spülsysteme in Hinblick auf die smear layer- bzw. Debrisentfernung realitätsnah untersuchen zu können, ist es erforderlich, die Versuche an extrahierten Zähnen durchzuführen, da nur dort die Erzeugung einer echten Schmierschicht bzw. von Debris gelingt. Andere Studien nutzen 
zur Untersuchung endodontischer Fragestellungen künstliche Wurzelkanäle in Kunststoffblöcken, um allen zu vergleichenden Systemen die gleichen Voraussetzungen zu bieten (Campos und del Rio 1990; Tronstad et al. 1986). Die Fragestellung dieser Studien setzte allerdings keine Bildung von smear layer oder Debris voraus. Die Zähne für die vorliegende Studie mussten folgende Kriterien erfüllen: Die mesiale Wurzel musste ein abgeschlossenes Wurzelwachstum aufweisen, einen Krümmungswinkel zwischen 20 und $40^{\circ}$ besitzen, eine Mindestlänge von $19 \mathrm{~mm}$ haben, sowie keine bereits angefangene oder abgeschlossene Wurzelkanalbehandlung zeigen. Für eine bessere Vergleichbarkeit wurde die Arbeitslänge einheitlich auf $18 \mathrm{~mm}$ festgelegt und durch Kürzen der einzelnen Zähne im koronalen Bereich auf 19 mm Zahnlänge gewährleistet. Da bei extrahierten Zähnen durch die nie identische Wurzelkanalanatomie keine echten Standardbedingungen geschaffen werden können, wurden nach Bestimmung der Krümmungswinkel und Randomisierung der Proben innerhalb der Versuchsgruppen einzelne Prüfkörper getauscht, um die Mittelwerte weitestgehend anzunähern. So war eine bessere Vergleichbarkeit der Gruppen gewährleistet. Während der experimentellen Phase, die die Präparation der Wurzelkanäle, deren Spülung sowie die Herstellung der Proben beinhaltete, mussten die Proben immer wieder gelagert werden. Um eine Beeinflussung der Zahnhartsubstanzbeschaffenheit oder aber der Oberflächen der Wurzelkanalwände möglichst gering zu halten, wurde auf den Einsatz von desinfizierenden Lösungen verzichtet. Die Lagerung der Proben erfolgte einzeln in Wasser in dicht verschlossenen Kunststoffbehältern. Aufgrund der Gruppengröße der vorliegenden Studie (=20) sind deren Ergebnisse mit anderen Studien nur bedingt vergleichbar. Die Gruppengrößen vergleichbarer Studien schwanken dabei zwischen 10 (Caron et al. 2010, Duque et al. 2016, Klyn et al. 2010; Munoz et al. 2012), über 15 bis 20 (Elnaghy et al. 2016, Rödig et al. 2010) bis zu 25-28 Wurzelkanälen (Al-Ali et al. 2012, Hülsmann et al. 2003).

Neben den verwendeten Materialien und Geräten ist laut Littman (1977) die „Performance“, also die Leistung bzw. die Ausführung des Zahnarztes, eine einflussreiche Variable bei der Wurzelkanalpräparation und -reinigung. So beschreibt er, dass die Fähigkeiten des Zahnarztes eine größere Rolle für die 
Sauberkeit spielen als die Präparationstechnik (Littman 1977). Um eine gute Vergleichbarkeit zwischen den Gruppen zu gewährleisten, wurden alle Maßnahmen wie Probenherstellung, Präparation und Spülung von lediglich einem Zahnarzt durchgeführt. Dabei wurde stets streng nach vorgegebenen Abläufen gearbeitet.

\subsubsection{Herstellung der Versuchskörper}

Die Herstellung der Versuchskörper in dieser Studie orientiert sich an der Methodik einer Vielzahl weiterer Studien. Das Muffelsystem zur Herstellung der Versuchskörper wurde erstmals von Bramante et al. (1987) beschrieben und später mehrfach modifiziert. Die Herstellung der Versuchskörper mit Hilfe des Bramante-Muffelsystems weist keine große Fehleranfälligkeit für die spätere Beurteilung der Proben auf und wird daher in der Diskussion nicht näher betrachtet.

\subsubsection{Präparation der Wurzelkanäle}

Die Präparation der mesiobukkalen Wurzelkanäle erfolgte ausschließlich mit dem BioRace-System. Dabei kam die Basis-Sequenz zum Einsatz, welche eine Präparation bis zur Größe .04/40 vorsieht. Dies ist die Mindestgröße, die für eine effektive Spülung bis in das apikale Drittel des Wurzelkanals erforderlich ist, eine Präparation bis zur ISO-Größe 60 bringt nach Ram (1977) keine Vorteile. Die Verwendung eines maschinellen NiTi-Systems zur Präparation der Wurzelkanäle in dieser Studie lag zum einen in einer besseren Vergleichbarkeit der Ergebnisse begründet, zum anderen im erhöhten Komfort bei der Präparation. Die Verwendung von NiTi-Systemen zur Wurzelkanalpräparation wird in der Literatur unterschiedlich bewertet. Eine der manuellen Präparation überlegene Reinigung ist ebenso zu finden (Hülsmann und Stryga 1993; Hülsmann et al. 1997; Tewari et al. 2016; Topcu et al. 2014) wie die Feststellung, dass es keinen signifikanten Unterschied zwischen maschineller sowie manueller Präparation gibt (Barrieshi-Nusair 2002; Kiran et al. 2016; Schäfer und Zapke 2000; Schirrmeister et al. 2006; Subramaniam et al. 2016). Auch Studien, die der manuellen Präparation eine überlegene Reinigung zusprechen, sind zu finden (Alam et al. 2006; Bueno et al. 2006; Gergi und Sabbagh 2007). Eine Kombination aus rotierender 
sowie anschließender manueller Präparation empfehlen Kiran et al. (2016). Bei der Präparation der Wurzelkanäle wurden alle Arbeitsabläufe streng eingehalten und auf eine gleichmäßige Präparation aller Versuchskörper geachtet. Die Präparation erfolgte dabei nicht Gruppe für Gruppe, sondern es wurde nach jedem präparierten Wurzelkanal ein Versuchskörper der nächsten Gruppe gewählt. Auch die spätere Spülung erfolgte nach diesem Muster. Sämtliche Parameter wie Drehmoment, Geschwindigkeit, Menge und Konzentration der Spülflüssigkeit sowie die Temperatur der Spülflüssigkeit wurden konstant beibehalten. Die Feilen stumpfen bei mehrfacher Benutzung von Kanal zu Kanal mehr und mehr ab und wurden daher nie länger als die vom Hersteller empfohlene Anzahl an 4 Wurzelkanälen benutzt, um Unterschiede in Konsistenz und Dicke der Schmierschicht zu vermeiden.

\subsubsection{Herstellung der Proben für das Rasterelektronenmikroskop}

Die Herstellung der Proben für das Rasterelektronenmikroskop unterlag ebenfalls einer genauen Abfolge von Arbeitsschritten. Die Herstellung der Proben bot jedoch von allen Arbeitsschritten die höchste Fehleranfälligkeit, da eine Verunreinigung der Proben nicht vollständig ausgeschlossen werden konnte. Die Herstellung setzte sich aus folgenden Schritten zusammen: Nach der Präparation und Spülung laut Protokoll wurde der präparierte Wurzelkanal mit einem Guttapercha-Stift der ISO Größe 40 verschlossen. Dabei wurde darauf geachtet, dass dieser auch das Foramen apicale ausfüllte, um eine Verunreinigung durch Schleifstaub zu verhindern. Anschließend erfolgte die Herstellung der Sägeschnitte. Dabei wurde der Versuchskörper (in Kunststoffblock eingebetteter Zahn) an zwei Stellen quer mit Hilfe einer Trennscheibe durchtrennt. Durch den mit Guttapercha ausgefüllten Wurzelkanal konnte so eine Verunreinigung der Wände weitestgehend ausgeschlossen werden. Die durch das Muffelsystem außen am Kunststoffblock befindlichen Rillen dienten dabei als Orientierung, dass die entstehenden Querschnitte aus den gleichen koronalen bzw. apikalen Abschnitten stammten. So entstanden zwei Querschnitte des Wurzelkanals, jeweils ein Querschnitt aus dem koronalen sowie aus dem apikalen Bereich. Im nächsten Schritt folgte die longitudinale Trennung des Querschnittspräparates in zwei Hälften. Dabei wurde wiederum mit 
größtmöglicher Vorsicht mit Hilfe einer Trennscheibe und unter Wasserkühlung von zwei gegenüberliegenden Seiten jeweils bis kurz vor das Wurzelkanallumen ein Sägeschnitt angelegt, das Lumen wurde jedoch nicht eröffnet. Mit Hilfe eines Heidemannspatels erfolgte die endgültige Trennung des Querschnittspräparates, so dass zwei Hälften entstanden, die jeweils den direkten Blick auf die Wurzelkanalwand ermöglichten. Anschließend wurde die Guttapercha vorsichtig mit Hilfe einer zahnärztlichen Sonde aus dem Wurzelkanalabschnitt entfernt. Dieses Vorgehen findet sich auch in ähnlichen Studien (Al-Ali et al. 2012; Arslan et al. 2016; Caron et al. 2010; Hülsmann et al. 2002; Stevens et al. 2006; Teixeira et al. 2005). Aufgrund der Anzahl der Arbeitsschritte und der nötigen Präzision zur Herstellung der Proben kann eine geringe Verunreinigung der Proben durch Schleifstaub von Kunststoff, Dentin und Wasser nicht völlig ausgeschlossen werden. Dies sollte bei der Bewertung der Ergebnisse bedacht werden. Weiterhin war die Möglichkeit zur Verunreinigung im apikalen Wurzelkanalanteil höher, da aufgrund des geringeren Durchmessers dort ein präzises Arbeiten schwerer war als im koronalen Abschnitt.

\subsection{Diskussion der Ergebnisse}

Die Einordnung der Ergebnisse in die Literatur wird durch verschiedene Faktoren erschwert. Unterschiedliche Studiendesigns, wechselnde Parameter, verschiedene Geräte, die Zahl der Proben sowie die stets unterschiedliche Performance des Behandlers sind nur einige Punkte, die eine Vergleichbarkeit der Studien erschweren. Zusätzlich kommt hinzu, dass in dieser Studie ausschließlich mit Natriumhypochlorit gespült wurde und nicht zusätzlich mit einem Chelator wie EDTA, um anorganische Bestandteile des smear layer aufzulösen, wie es in vielen vergleichbaren Studien der Fall ist (Ballal et al. 2016; Calt und Serper 2002; Conde et al. 2016; Grawehr et al. 2003; Guo et al. 2014; Soares et al. 2010; Teixeira et al. 2005; Zaparolli et al. 2012). Der Verzicht auf die Spülung mit einem Chelator liegt in dieser Studie in der Fragestellung begründet, ob eine Verbesserung der Entfernung des smear layer allein durch eine neuartige Aktivierungsmethode verbessert werden kann. Durch die ausschließliche Spülung mit $\mathrm{NaOCl}$ war keine 
vollständige Entfernung des smear layer zu erwarten. Allgemein gilt, dass neben der Menge der Spülflüssigkeit auch die Größe der apikalen Präparation wichtigen Einfluss auf die Ergebnisse nimmt. Diese sollte bei mindestens ISO 35 liegen (Schäfer 2007). Großvolumige Spülungen sorgen für eine bessere Sauberkeit der Wurzelkanalwand als kleinvolumige Spülungen (Byström und Sundqvist 1985; Schäfer 2007). Die in dieser Studie verwendeten Mengen bei der Spülung entsprechen dem aktuellen klinischen Standard (Byström und Sundqvist 1985; Hülsmann 2006; Schäfer 2007).

\subsubsection{Reinigungseffektivität der Handspülung}

Die Ausführung der dynamischen Handspülung erfolgte in der vorliegenden Studie mit Hilfe einer Einmalspülspritze aus Kunststoff mit einem Fassungsvermögen von $5 \mathrm{ml}$ sowie einer endodontischen Spülspitze mit einem Außendurchmesser von 0,35 mm, was der ISO-Größe 35 entspricht. Die Größe der Spülspitze passte somit zur apikalen Aufbereitungsgröße von ISO 40, was nach Ram (1977) sowie Schäfer (2007) für eine effektive apikale Desinfektion notwendig sei, da die Wirkung bei der dynamischen Handspülung wenige Millimeter vor der Spitze der Spülnadel endet. Hsieh et al. (2007) untersuchten den Zusammenhang zwischen apikaler Präparationsgröße und verwendeter Nadelgröße und stellten fest, dass eine gute apikale Reinigung bereits ab ISO-Größe 30 gelingen kann. Um auch bei der Handspülung einen hydrodynamischen Effekt zu erzielen, sei es nötig, während der Spülung leichte Auf- und Abwärtsbewegungen von einigen Millimetern auszuführen (Park et al. 2013). Damit wird sichergestellt, dass neben einer konstanten Flussrate die gewünschte Hydrodynamik entsteht, außerdem wird somit ein apikales Überpressen der Spülflüssigkeit vermieden (Park et al. 2013). Während der manuellen Spülung ist jederzeit darauf zu achten, dass passiv gespült wird. Dies bedeutet, dass langsam und ohne Druck gearbeitet werden muss. Diese Faktoren wurden bei der Spülung im Rahmen dieser Studie beachtet und streng eingehalten. Die Ergebnisse der dynamischen Handspülung zeigen sich in der vorliegenden Studie konstant. Bei der Entfernung von Debris lassen sich keine signifikanten Unterschiede zwischen koronalem und apikalem Wurzelkanalabschnitt feststellen. Betrachtet man die Entfernung des Debris gesamt für die Gruppe der 
dynamischen Handspülung, so ergibt sich eine zu $55 \%$ sehr gute oder gute Reinigung der Kanalwände. Im Vergleich mit den aktivierten Gruppen zeigt die dynamische Handspülung hier die schlechtesten Ergebnisse, da die prozentualen Ergebnisse der aktivierten Gruppen für die Scores 1 und 2 deutlich höher, jedoch nicht im statistisch signifikanten Bereich liegen. Bei der Entfernung von smear layer zeigt die dynamische Handspülung etwas deutlichere Unterschiede bei den Ergebnissen. Koronal gelingt die Entfernung der Schmierschicht hier deutlich besser als im apikalen Wurzelkanalanteil, statistisch signifikante Unterschiede ließen sich jedoch auch hier nicht finden. Bei der Betrachtung der Entfernung des smear layer gesamt für die Gruppe der dynamischen Handspülung fällt auch hier wieder ein Vorteil der aktivierten Gruppen auf. Diese Ergebnisse decken sich mit anderen Studien, die der dynamischen Handspülung eine weniger effektive Reinigungsleistung gegenüber aktivierten Systemen bescheinigen (Arslan et al. 2014; Çapar und Ari Aydinbelge 2014). Auch zeigen sich nach Anwendung einer ultraschallaktivierten Spülung mehr saubere Seitenkanäle sowie offen liegende Dentintubuli als bei der Handspülung (Chen et al. 2016). Die dynamische Handspülung ist in der vorliegenden Studie die am wenigsten effektive Methode zur Entfernung von Debris sowie von smear layer. Dies lässt sich jedoch nur prozentual belegen, eine statistisch signifikante Überlegenheit der aktivierten Gruppen ergab sich nicht. Generell ist die Effektivität der dynamischen Handspülung als gut einzustufen und findet daher im klinischen Alltag, sicherlich auch aufgrund ihrer Einfachheit und geringen Anforderungen an Kosten und Material, weiterhin zu Recht Beachtung und Verwendung.

\subsubsection{Reinigungseffektivität des EndoActivator®}

Die Aktivierung mit dem EndoActivator ${ }^{\circledR}$ erfolgte in dieser Studie mit einer Spülspitze der Größe „Large“ (Activator Tip 35/.04). Auch hier galt es darauf zu achten, dass die ISO-Größe des Aktivierungssystems zur apikalen Aufbereitungsgröße passte. Die Aktivierung erfolgte jeweils mit der Einstellung von $10.000 \mathrm{cpm}$ (Counts per minute), welche von Herstellerseite für die Aktivierung der Spülflüssigkeit empfohlen wird. Die Dauer der Aktivierung betrug dabei 20 Sekunden. Niu et al. (2014) empfehlen für die 
besten Ergebnisse mit dem EndoActivator ${ }^{\circledR} 30$ Sekunden Aktivierungszeit. Möglicherweise wäre bei längerer Aktivierung eine Verbesserung der Ergebnisse zu beobachten. Mit Hilfe der schallaktivierten Spülung mit dem EndoActivator® fällt bei der Beurteilung der Ergebnisse zunächst auf, dass prozentual höhere Werte als bei der dynamischen Handspülung erreicht wurden. Der EndoActivator ${ }^{\circledR}$ erreicht in $85 \%$ der Proben eine gute oder sehr gute Reinigung der Wurzelkanalwände von Debris. Auffallend ist hierbei, dass die Reinigung koronal und apikal gleich gut gelingt. Dies steht im Gegensatz zu den Ergebnissen von Elnaghy et al. (2016), die dem EndoActivator® koronal eine bessere Reinigungswirkung zusprechen. Prozentual gesehen gelingt die Reinigung mit dem EndoActivator ${ }^{\circledR}$ besser als mit der dynamischen Handspülung. Dies stimmt mit der Studie von Elnaghy et al. (2016) überein. Die in dieser Studie ermittelten Werte liegen aber unterhalb des statistischen Signifikanzniveaus. Die Entfernung von smear layer erzielt im Vergleich zum Debris deutlich schlechtere Ergebnisse. Die Kanalwände wurden hier nur in $25 \%$ der Fälle mit einem Score von 1 oder 2 bewertet. Dies entspricht dem schlechtesten Ergebnis innerhalb der aktivierten Gruppen. Apikal erreicht der EndoActivator $®$ mit 12,5 \% deutlich weniger saubere Wurzelkanalwände als in den koronalen Abschnitten mit 35 \%. UrozTorres et al. (2010) beschrieben, dass der EndoActivator® nicht in der Lage sei, die Entfernung des smear layer im Vergleich mit der konventionellen Handspülung zu verbessern. Zu diesem Ergebnis kamen auch Klyn et al. (2010), welche keinen Unterschied in der Effektivität der Reinigung zwischen dynamischer Handspülung, passiver Ultraschallreinigung sowie dem EndoActivator® feststellen konnten. In dieser Studie ist eine leichte Tendenz zur verbesserten Entfernung des smear layer mit dem EndoActivator ${ }^{\circledR} \mathrm{zu}$ erkennen, jedoch lassen sich auch diese Ergebnisse erneut nur prozentual und nicht statistisch belegen. Auch Arslan et al. (2016) zeigten, dass der EndoActivator® die Entfernung der Schmierschicht im Gegensatz zur dynamischen Handpülung verbessern könne. Saubere und von smear layer befreite Kanäle lassen sich nach Uroz-Torres et al. (2010) nur durch die zusätzliche Verwendung von EDTA erreichen. Übereinstimmend ist das Ergebnis, dass die Entfernung des smear layer im koronalen Anteil besser gelingt als in den apikalen Wurzelkanalabschnitten. 


\subsubsection{Reinigungseffektivität des IrriSafe ${ }^{T M}$}

Bereits 1976 stellte Martin fest, dass die passive Ultraschallaktivierung einen verbessernden Effekt auf die Reinigung der Wurzelkanäle haben kann. Dabei setzte er jedoch die Verwendung von $\mathrm{NaOCl}$ voraus, um eine effektive Reinigung zu ermöglichen (Martin 1976). Die passive Ultraschallaktivierung in der vorliegenden Studie erfolgte mit dem IrriSafe ${ }^{\mathrm{TM}}$-System. Zur Anwendung kam die Spülspitze der Größe 20/21 in Verbindung mit dem Ultraschall-Mikromotor VDW Ultra. Die Aktivierung der Spülflüssigkeit erfolgte mit einer Frequenz von $30 \mathrm{KHz}$. Die Reinigung der Wurzelkanalwände gelang in $72,5 \%$ der Fälle gut oder sehr gut. Jedoch zeigt die passive Ultraschallaktivierung innerhalb der aktivierten Gruppen das mit geringem Abstand schlechteste Ergebnis. In den koronalen Proben erreicht die PUI einen Anteil von 70 \% an sauberen Wurzelkanalwänden, im apikalen Bereich liegt der Wert mit 75 \% noch leicht höher. Die Tendenz, auch im apikalen Bereich eine gute und unter Umständen bessere Reinigung als koronal zu erzielen, wurde bereits in anderen Studien erwähnt (Munoz und CamachoCuadra 2012; Prado et al. 2016). Die Ergebnisse sind hier allerdings nur prozentual belegbar und nicht statistisch nachweisbar. Auch der Vergleich zur konventionellen Handspülung legt nahe, dass die PUI besser reinigt, jedoch liegt auch hier nur ein geringer prozentualer Vorsprung vor. Die beiden Methoden können also nach den Ergebnissen als gleichwertig angesehen werden. Auch de Castro et al. (2016) stellten fest, dass die konventionelle Handspülung und die passive Ultraschallspülung eine ähnliche Effektivität zeigen. Widersprüchlich dazu stehen die Ergebnisse von Duque et al. (2016), nach denen die PUI eine bessere Reinigung als die konventionelle Spülung bewirkt, jedoch nur unter der Voraussetzung, dass drei aufeinanderfolgende Spüldurchgänge durchgeführt werden.

Einen Vergleich der ultraschallaktivierten Spülung mit der schallaktivierten Spülung stellten u.a. Jiang et al. (2010) an. Ihre Ergebnisse belegen, dass die PUI eine signifikant bessere Reinigung ermöglichte als die schallbasierte Aktivierung. Diese Ergebnisse sind jedoch nicht ohne Weiteres mit der vorliegenden Studie vergleichbar, da andere Systeme verwendet wurden. Erwartungsgemäß gelingt die Reinigung von smear layer deutlich schlechter 
als von Debris. In 37,5 \% erreicht IrriSafe ${ }^{\mathrm{TM}}$ eine Reinigung, die mit den Scores 1 oder 2 bewertet wurde. Dies ist prozentual das beste Ergebnis aller Gruppen. Koronal konnte die Hälfte aller Proben gut bis sehr gut von smear layer befreit werden. Apikal liegt der Wert mit $25 \%$ nur halb so hoch. Dies deckt sich mit den Ergebnissen von Castelo-Baz et al. (2016), die der Ultraschallaktivierung apikal eine schlechtere Reinigung bescheinigen.

\subsubsection{Reinigungseffektivität des EDDY ${ }^{\circledR}$}

Die Spülspitze EDDY ${ }^{\circledR}$ ist nur in einer Universalgröße erhältlich. Aufgrund ihrer Konstruktion ist es aber möglich, sie bei unterschiedlichsten apikalen Präparationsgrößen bis auf Arbeitslänge in den Wurzelkanal einführen zu können. In der vorliegenden Studie konnte die Spülspitze problemlos auf die entsprechende Arbeitslänge abzüglich eines Millimeters angewendet werden. EDDY $^{\circledR}$ stellt neben dem EndoActivator ${ }^{\circledR}$ das zweite schallbasierte Aktivierungssystem in der vorliegenden Studie dar. Die Aktivierung erfolgt dabei aber mit einer deutlich höheren Frequenz als beim EndoActivator $\circledast$. Die Vergleichbarkeit der mit EDDY ${ }^{\circledR}$ erzielten Ergebnisse in dieser Studie mit der Literatur kann bisher nur in kleinem Umfang erfolgen, da EDDY ${ }^{\circledR}$ noch nicht Gegenstand vieler Untersuchungen war. In der vorliegenden Studie ordnen sich die mit $\operatorname{EDDY}^{\circledR}$ erzielten Ergebnisse auf dem Niveau der anderen aktivierten Gruppen ein. So erreichten 80 \% der gesamten Proben, die mit $E D D Y^{\circledR}$ von Debris gereinigt wurden, einen Score von 1 oder 2. Nur die Gruppe des EndoActivators ${ }^{\circledR}$ zeigt hier prozentual noch leicht bessere Ergebnisse. Koronal zeigen 85 \% der Proben saubere Wurzelkanalwände, apikal beträgt dieser Wert noch $75 \%$. Wie bei den anderen Gruppen zeigen sich bei der Untersuchung der Entfernung des smear layer erneut deutlich schlechtere Ergebnisse. 35 \% der Proben wurden hier als sauber bewertet. Auch hier ist das Ergebnis dadurch zu erklären, dass auf den Einsatz von EDTA verzichtet wurde. Koronal erreicht EDDY ${ }^{\circledR}$ dabei mit $60 \%$ sauberer Proben prozentual das beste Ergebnis aller Gruppen. Apikal werden mit EDDY $^{\circledR}$ nur $10 \%$ der Proben ausreichend von smear layer befreit. Der Vergleich der Ergebnisse beschränkt sich bisher auf drei verwandte Studien. Urban et al. (2017) untersuchten ähnlich zur vorliegenden Studie die Effektivität bei der Entfernung von Debris sowie smear layer mit 
unterschiedlichen Aktivierungssystemen. EDDY ${ }^{\circledR}$, der EndoActivator ${ }^{\circledR}$ sowie die passive Ultraschallspülung konnten signifikant mehr Debris entfernen als die dynamische Handspülung (Urban et al. 2017). Dieses Ergebnis steht im Gegensatz zur vorliegenden Studie, bei der kein signifikanter Unterschied zwischen aktivierten Methoden und nicht aktivierter Spülung festgestellt werden konnte. Bei der Entfernung von smear layer sei innerhalb der aktivierten Gruppen kein signifikanter Unterschied festzustellen, jedoch sei mit EDDY ${ }^{\circledR}$ sowie der passiven Ultraschallspülung eine der Handspülung signifikant überlegene Reinigung von smear layer möglich (Urban et al. 2017). Ein Erklärungsansatz für diese unterschiedlichen Ergebnisse liegt möglicherweise darin, dass in der Studie von Urban et al. ausschließlich gerade Wurzelkanäle untersucht wurden. Eine weitere Studie untersuchte die Wirkung der Reinigung mit EDDY ${ }^{\circledR}$ in Bezug auf die Reduktion unterschiedlicher Bakterien im Wurzelkanalsystem. Die generell höchste Reduktion von Mikroorganismen konnte dabei durch die Anwendung von EDDY ${ }^{\circledR}$ in Verbindung mit $\mathrm{NaOCl}$ erreicht werden (Neuhaus et al. 2016). Besonders in gekrümmten Kanälen sei EDDY ${ }^{\circledR}$ mindestens so effektiv wie die passive Ultraschallspülung, jedoch sei auch dort kein signifikanter Unterschied festzustellen (Neuhaus et al. 2016). Mit der Entfernung von medikamentösen Kalziumhydroxideinlagen aus Wurzelkanälen sowie künstlich erzeugten Wurzelkanalgruben beschäftigten sich Hergt et al. (2017). Dabei stellten sie fest, dass die Entfernung mit EDDY ${ }^{\circledR}$ sowie der passiven Ultraschallspülung signifikant besser gelang als per Handspülung oder EndoActivator®. 


\section{$7 \quad$ Zusammenfassung}

Für eine langfristig erfolgreiche Wurzelkanalbehandlung sind die Abfolge bestimmter Arbeitsschritte, das Zusammenspiel verschiedenster Techniken, Geräte sowie Materialien und nicht zuletzt die Erfahrung des Zahnarztes von entscheidender Bedeutung. Einen sehr hohen Stellenwert nimmt dabei die Desinfektion und Reinigung des Wurzelkanalsystems ein. Dabei bietet der heutige Stand der Forschung eine Vielzahl an Möglichkeiten, wie Spülungen appliziert und aktiviert werden können. Diese Studie zeigt nur eine kleine Auswahl von heutigen Möglichkeiten, die Wurzelkanalreinigung durchzuführen. In dieser Studie wurde die Effektivität von vier unterschiedlichen Spülmethoden untersucht. Hauptaugenmerk lag auf einem zum Studienzeitpunkt erst relativ kurz verfügbaren System, dessen Effektivität in das Umfeld anderer etablierter Systeme eingeordnet werden sollte. Der Vergleich fand dabei zwischen der dynamischen Handspülung, dem EndoActivator ${ }^{\circledR}$, der passiven Ultraschallspülung sowie der Spülung mit EDDY ${ }^{\circledR}$ statt. Untersucht wurden dabei folgende Parameter:

- Sauberkeit der Wurzelkanalwand in Hinsicht auf Debris sowie smear layer

- Sauberkeit in verschiedenen Abschnitten des Wurzelkanals (koronal und apikal).

Zu diesem Zweck wurden 90 extrahierte humane Unterkiefermolaren verwendet, die verschiedene Voraussetzungen erfüllen mussten. Die mesialen Wurzeln mussten vollständig intakt sein und ein abgeschlossenes Wurzelwachstum aufweisen. Der Krümmungswinkel der mesialen Wurzeln sollte 20 bis $40^{\circ}$ betragen und die Zähne mussten eine Mindestlänge von 19 $\mathrm{mm}$ aufweisen. Weiterhin durfte kein s-förmiger Kanalverlauf vorhanden sein. Nach einer randomisierten Einteilung in die Versuchsgruppen wurden die Zähne mit Hilfe des Bramante-Muffelsystems vorbereitet und im Anschluss mit dem BioRace NiTi-System unter Anwendung der Basis-Sequenz auf eine Größe von 40/.04 präpariert. Die Spülung erfolgte stets streng nach Protokoll, sowohl zwischen den Arbeitsschritten als auch während der 
Abschlussspülung. Nach Herstellung der Querschnittspräparate erfolgte deren Auswertung am Rasterelektronenmikroskop sowohl für Debris als auch für smear layer.

Von den vier getesteten Systemen ist keines in der Lage, Wurzelkanäle vollständig von Debris oder smear layer zu reinigen. Dabei ist erneut zu erwähnen, dass in Bezug auf smear layer die Effektivität der Reinigung den Erwartungen entsprach, da auf den Einsatz eines Chelators bei der Spülung verzichtet wurde. Der Vergleich zwischen allen Gruppen zeigt, dass keines der untersuchten Systeme eine den anderen überlegene Reinigungswirkung aufweist. Es zeigten sich lediglich prozentuale Vorteile, die aber in keinem Vergleich statistisch signifikante Ergebnisse lieferten. Tendenziell ließ sich ein Vorteil der aktivierten Systeme gegenüber der dynamischen Handspülung erkennen.

Die Anwendung aktivierter Spülsysteme scheint gegenüber der dynamischen Handspülung tendenziell eine bessere Reinigung zu erzielen, jedoch ließ sich kein statistisch signifikanter Unterschied zwischen den Gruppen feststellen. EDDY - als zum Studienzeitpunkt relativ neues System - ordnet sich auf dem Niveau der anderen aktivierten Systeme in die Ergebnisse ein. Es kann somit geschlussfolgert werden, dass eine aktivierte Spülung Vorteile gegenüber der konventionellen dynamischen Handspülung bietet. In der täglichen Routine können alle verwendeten Systeme für eine ausreichende Reinigung der Wurzelkanäle Anwendung finden. Der Vergleich mit anderen Studien legt nahe, dass die Verwendung einer Kombination von $\mathrm{NaOCl}$ und EDTA essenziell ist für eine bestmögliche Entfernung des smear layer und somit unabhängig von der Spülmethode - immer Anwendung finden sollte. Zu überprüfen bleibt ebenfalls, ob eine Aktivierung eines Chelators mit einer der getesteten Aktivierungshilfen Vorteile gegenüber der passiven Applikation ergibt. 


\section{Anhang}

\subsection{Ergebnisse: Scores}

\begin{tabular}{|c|c|c|c|c|c|}
\hline & Zahn & $\begin{array}{l}\text { Debris } \\
\text { koronal }\end{array}$ & $\begin{array}{l}\text { Debris } \\
\text { apikal }\end{array}$ & $\begin{array}{c}\text { smear } \\
\text { layer } \\
\text { koronal }\end{array}$ & $\begin{array}{l}\text { smear } \\
\text { layer } \\
\text { apikal }\end{array}$ \\
\hline \multirow[t]{20}{*}{ Gruppe 1} & 1 & 1 & 3 & 1 & 4 \\
\hline & 2 & 2 & 2 & 3 & 3 \\
\hline & 3 & 3 & 2 & 3 & 3 \\
\hline & 4 & 3 & 2 & 4 & 4 \\
\hline & 5 & 3 & 2 & 4 & 4 \\
\hline & 6 & 3 & 2 & 5 & 5 \\
\hline & 7 & 2 & 2 & 3 & 4 \\
\hline & 8 & 4 & 2 & 5 & 3 \\
\hline & 9 & 3 & 3 & 4 & 5 \\
\hline & 10 & 2 & 5 & 3 & 5 \\
\hline & 11 & 2 & 2 & 3 & 3 \\
\hline & 12 & 3 & 4 & 5 & 5 \\
\hline & 13 & 2 & 2 & 1 & 3 \\
\hline & 14 & 3 & 1 & 2 & 3 \\
\hline & 15 & 1 & 5 & 1 & 5 \\
\hline & 16 & 1 & 3 & 1 & 2 \\
\hline & 17 & 3 & 4 & 5 & 4 \\
\hline & 18 & 1 & 1 & 2 & 4 \\
\hline & 19 & 2 & 4 & 3 & 5 \\
\hline & 20 & 5 & 2 & 5 & 4 \\
\hline
\end{tabular}

Tabelle 12: Ergebnisse Gruppe 1: Dynamische Handspülung

\begin{tabular}{|l|c|c|c|c|c|}
\hline & Zahn & $\begin{array}{c}\text { Debris } \\
\text { koronal }\end{array}$ & $\begin{array}{c}\text { Debris } \\
\text { apikal }\end{array}$ & $\begin{array}{c}\text { smear } \\
\text { layer } \\
\text { koronal }\end{array}$ & $\begin{array}{c}\text { smear } \\
\text { layer } \\
\text { apikal }\end{array}$ \\
\hline Gruppe 2 & 21 & 1 & 2 & 2 & 4 \\
\cline { 2 - 6 } & 22 & 1 & 1 & 1 & 3 \\
\cline { 2 - 6 } & 23 & 2 & 1 & 3 & 3 \\
\cline { 2 - 6 } & 24 & 2 & 2 & 2 & 3 \\
\cline { 2 - 6 } & 25 & 1 & 3 & 4 & 5 \\
\cline { 2 - 6 } & 26 & 1 & 1 & 2 & 1 \\
\cline { 2 - 6 } & 27 & 1 & 5 & 2 & 5 \\
\hline
\end{tabular}




\begin{tabular}{|c|c|c|c|c|c|}
\hline & 28 & 1 & 2 & 2 & 4 \\
\cline { 2 - 6 } & 29 & 2 & 1 & 3 & 3 \\
\cline { 2 - 6 } & 30 & 2 & 2 & 3 & 2 \\
\cline { 2 - 6 } & 31 & 3 & 2 & 3 & 3 \\
\hline 32 & 1 & 1 & 4 & 4 \\
\hline & 33 & 2 & 2 & 3 & 5 \\
\cline { 2 - 6 } & 34 & 2 & 2 & 3 & 3 \\
\cline { 2 - 6 } & 35 & 2 & 3 & 3 & 3 \\
\hline 36 & 4 & 1 & 4 & 3 \\
\hline & 37 & 2 & 2 & 4 & 3 \\
\cline { 2 - 6 } & 38 & 2 & 1 & 4 & 2 \\
\cline { 2 - 6 } & 39 & 2 & 2 & 2 & 3 \\
\cline { 2 - 6 } & 40 & -- & 2 & -- & 4 \\
\hline
\end{tabular}

Tabelle 13: Ergebnisse Gruppe 2: EndoActivator®

\begin{tabular}{|c|c|c|c|c|c|}
\hline & Zahn & $\begin{array}{c}\text { Debris } \\
\text { koronal }\end{array}$ & $\begin{array}{l}\text { Debris } \\
\text { apikal }\end{array}$ & $\begin{array}{c}\text { smear } \\
\text { layer } \\
\text { koronal }\end{array}$ & $\begin{array}{l}\text { smear } \\
\text { layer } \\
\text { apikal }\end{array}$ \\
\hline \multirow{20}{*}{ Gruppe 3} & 41 & 2 & 1 & 3 & 3 \\
\hline & 42 & 3 & 2 & 2 & 2 \\
\hline & 43 & 2 & 2 & 1 & 2 \\
\hline & 44 & 1 & 1 & 2 & 1 \\
\hline & 45 & 2 & 3 & 4 & 3 \\
\hline & 46 & 4 & 2 & 2 & 1 \\
\hline & 47 & 3 & 5 & 4 & 4 \\
\hline & 48 & 1 & 2 & 1 & 4 \\
\hline & 49 & 2 & 2 & 4 & 3 \\
\hline & 50 & 2 & 1 & 5 & 3 \\
\hline & 51 & 1 & 1 & 1 & 3 \\
\hline & 52 & 1 & 2 & 3 & 3 \\
\hline & 53 & 4 & 3 & 5 & 4 \\
\hline & 54 & 3 & 5 & 3 & 5 \\
\hline & 55 & 3 & 3 & 2 & 4 \\
\hline & 56 & 2 & 1 & 2 & 2 \\
\hline & 57 & 1 & 2 & 3 & 4 \\
\hline & 58 & 1 & 2 & 2 & 4 \\
\hline & 59 & 2 & 2 & 3 & 3 \\
\hline & 60 & 1 & 2 & 1 & 3 \\
\hline
\end{tabular}

Tabelle 14: Ergebnisse Gruppe 3: IrriSafe ${ }^{\text {TM }}$ (Passive Ultraschallspülung)

\begin{tabular}{|c|c|c|c|c|c|}
\hline & Zahn & $\begin{array}{c}\text { Debris } \\
\text { koronal }\end{array}$ & $\begin{array}{c}\text { Debris } \\
\text { apikal }\end{array}$ & $\begin{array}{c}\text { smear } \\
\text { layer } \\
\text { koronal }\end{array}$ & $\begin{array}{c}\text { smear } \\
\text { layer } \\
\text { apikal }\end{array}$ \\
\hline Gruppe 4 & 61 & 1 & 2 & 2 & 3 \\
\cline { 2 - 6 } & 62 & 1 & 2 & 1 & 3 \\
\cline { 2 - 6 } & 63 & 3 & 5 & 3 & 5 \\
\cline { 2 - 6 } & 64 & 3 & 2 & 3 & 3 \\
\hline
\end{tabular}




\begin{tabular}{|c|c|c|c|c|c|}
\hline \multirow{4}{*}{} & 65 & 2 & 1 & 2 & 3 \\
\cline { 2 - 6 } & 66 & 2 & 5 & 1 & 5 \\
\hline 67 & 2 & 4 & 3 & 4 \\
\hline & 68 & 1 & 2 & 2 & 4 \\
\hline 69 & 1 & 1 & 2 & 3 \\
\hline & 70 & 1 & 2 & 2 & 2 \\
\hline 71 & 2 & 3 & 3 & 3 \\
\hline 72 & 1 & 3 & 2 & 5 \\
\hline & 73 & 3 & 2 & 4 & 3 \\
\hline & 74 & 1 & 2 & 3 & 3 \\
\hline 75 & 1 & 2 & 3 & 3 \\
\hline 76 & 2 & 2 & 2 & 3 \\
\hline 77 & 2 & 2 & 2 & 4 \\
\hline & 78 & 2 & 1 & 3 & 1 \\
\hline 79 & 2 & 1 & 1 & 3 \\
\hline & 70 & 2 & 2 & 1 & 3 \\
\hline
\end{tabular}

Tabelle 15: Ergebnisse Gruppe 4: EDDY ${ }^{\circledR}$

\begin{tabular}{|c|c|c|c|c|c|}
\hline \multirow{5}{*}{ Gruppe 5 } & Zahn & $\begin{array}{c}\text { Debris } \\
\text { koronal }\end{array}$ & $\begin{array}{c}\text { Debris } \\
\text { apikal }\end{array}$ & $\begin{array}{c}\text { smear } \\
\text { layer } \\
\text { koronal }\end{array}$ & $\begin{array}{c}\text { smear } \\
\text { layer } \\
\text { apikal }\end{array}$ \\
\cline { 2 - 6 } & 81 & 2 & 3 & 3 & 4 \\
\cline { 2 - 6 } & 82 & 1 & 5 & 1 & 5 \\
\cline { 2 - 6 } & 83 & 5 & 2 & 5 & 4 \\
\hline 84 & 5 & 5 & 5 & 5 \\
\hline & 85 & 2 & 5 & 2 & 5 \\
\cline { 2 - 6 } & 86 & 1 & 5 & 1 & 5 \\
\cline { 2 - 6 } & 87 & 2 & 1 & 3 & 3 \\
\cline { 2 - 6 } & 88 & 2 & 5 & 3 & 5 \\
\cline { 2 - 6 } & 89 & 4 & 4 & 4 & 4 \\
\cline { 2 - 6 } & 90 & 5 & 2 & 5 & 4 \\
\hline
\end{tabular}

Tabelle 16: Ergebnisse Gruppe 5: Kontrollgruppe 


\subsection{Statistische Auswertung}

\subsubsection{Box-Plots}

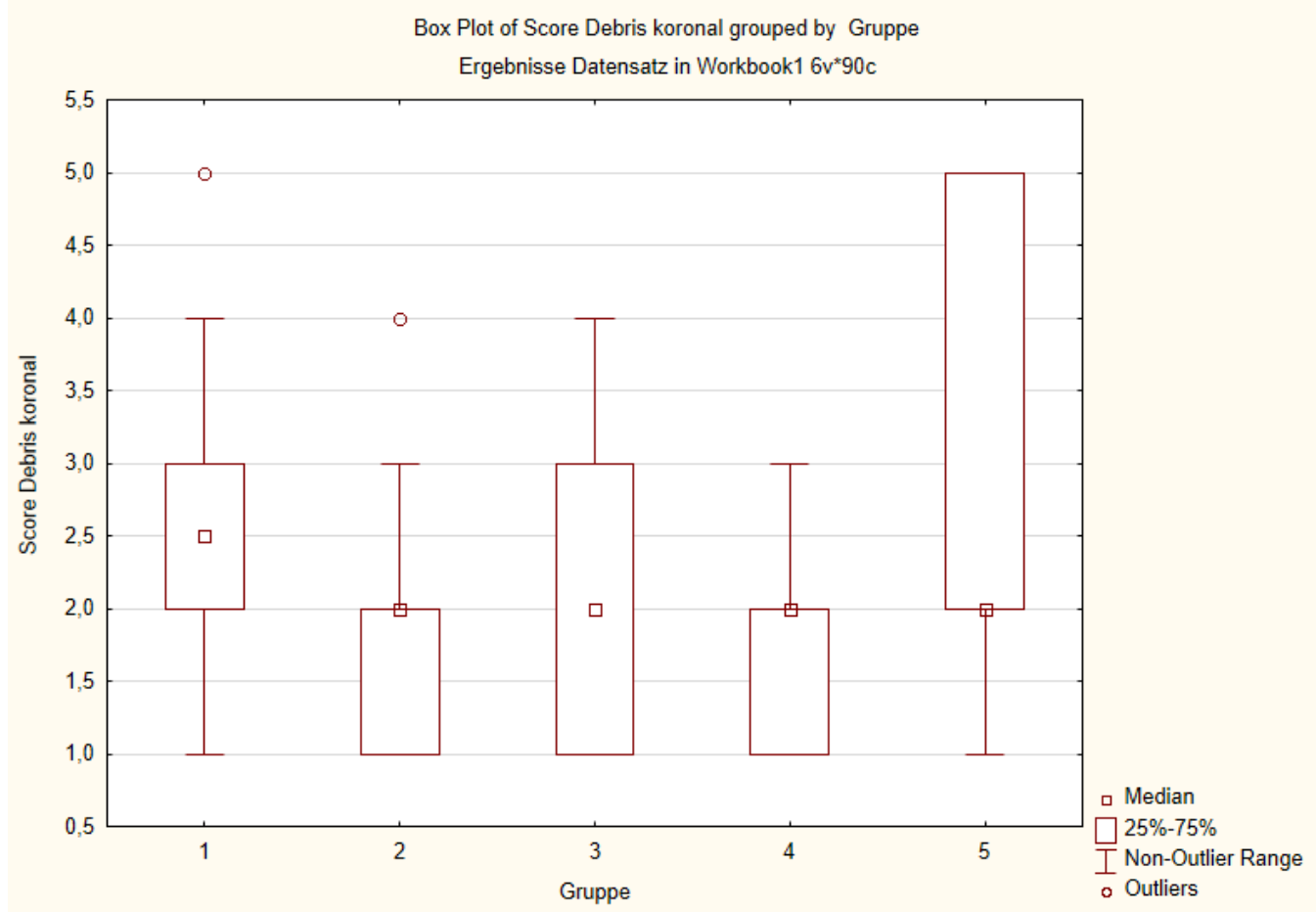

Abbildung 38: Box-Plot der koronalen Debris-Scores

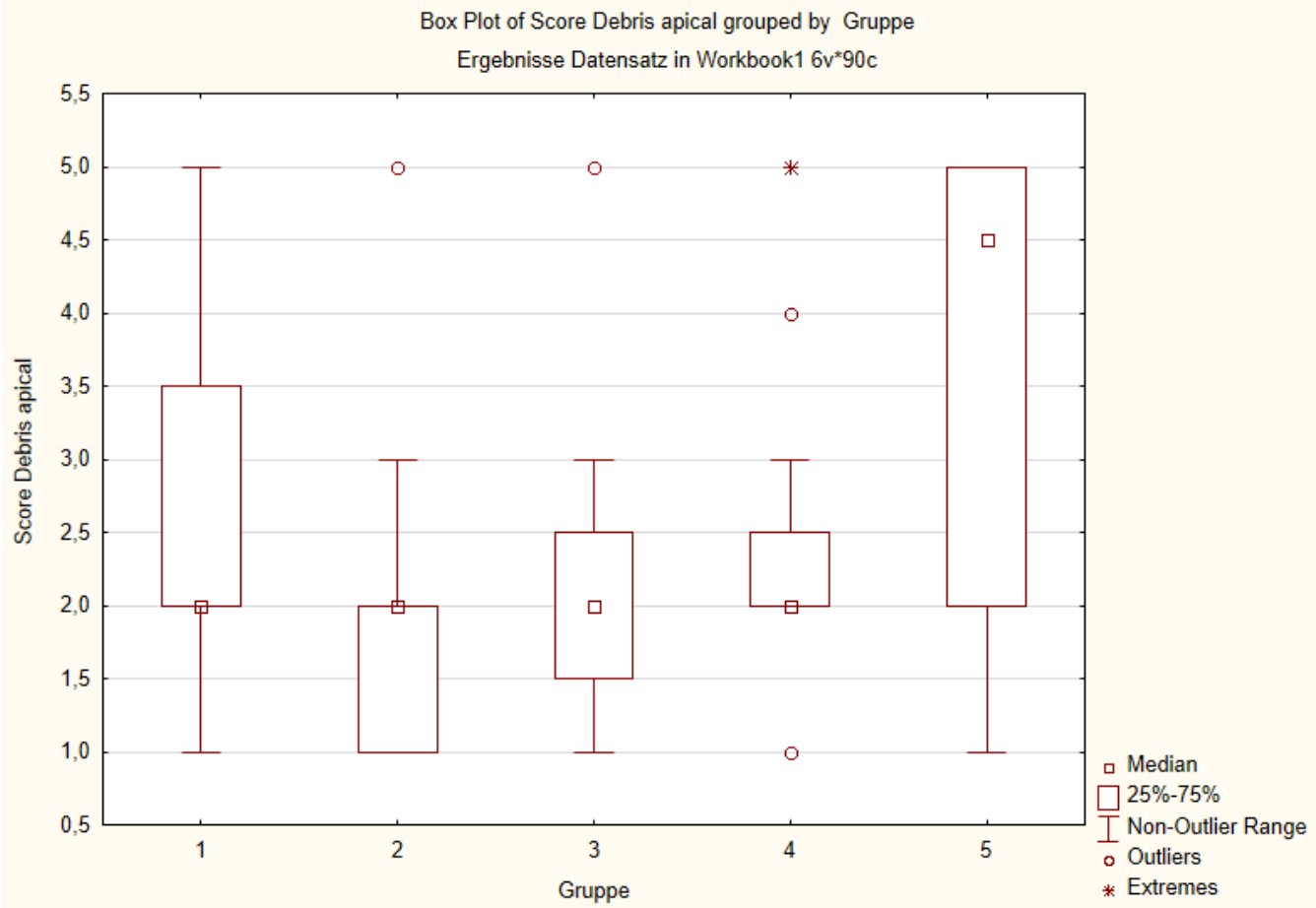

Abbildung 39: Box-Plot der apikalen Debris-Scores 


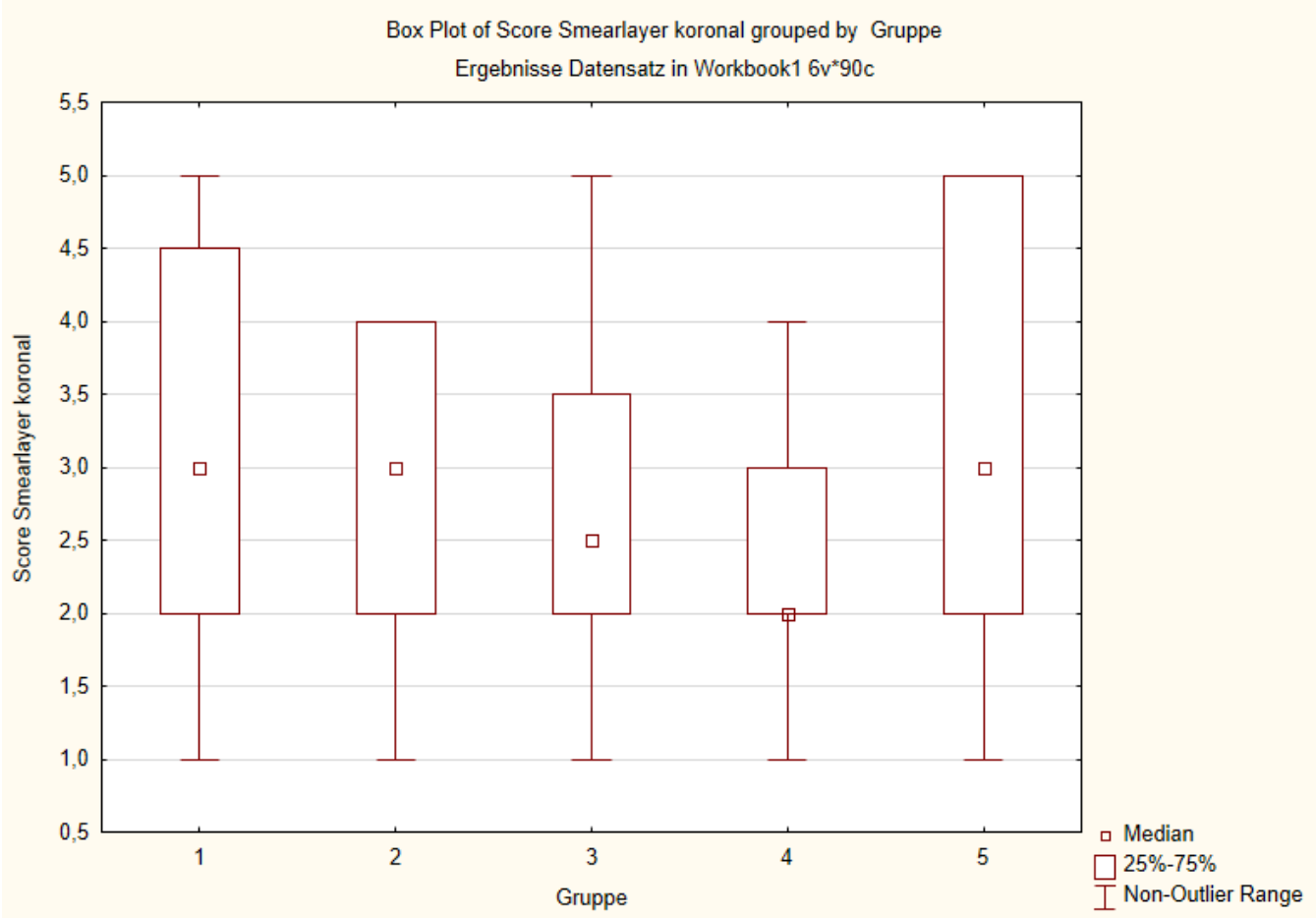

Abbildung 40: Box-Plot der koronalen smear layer-Scores

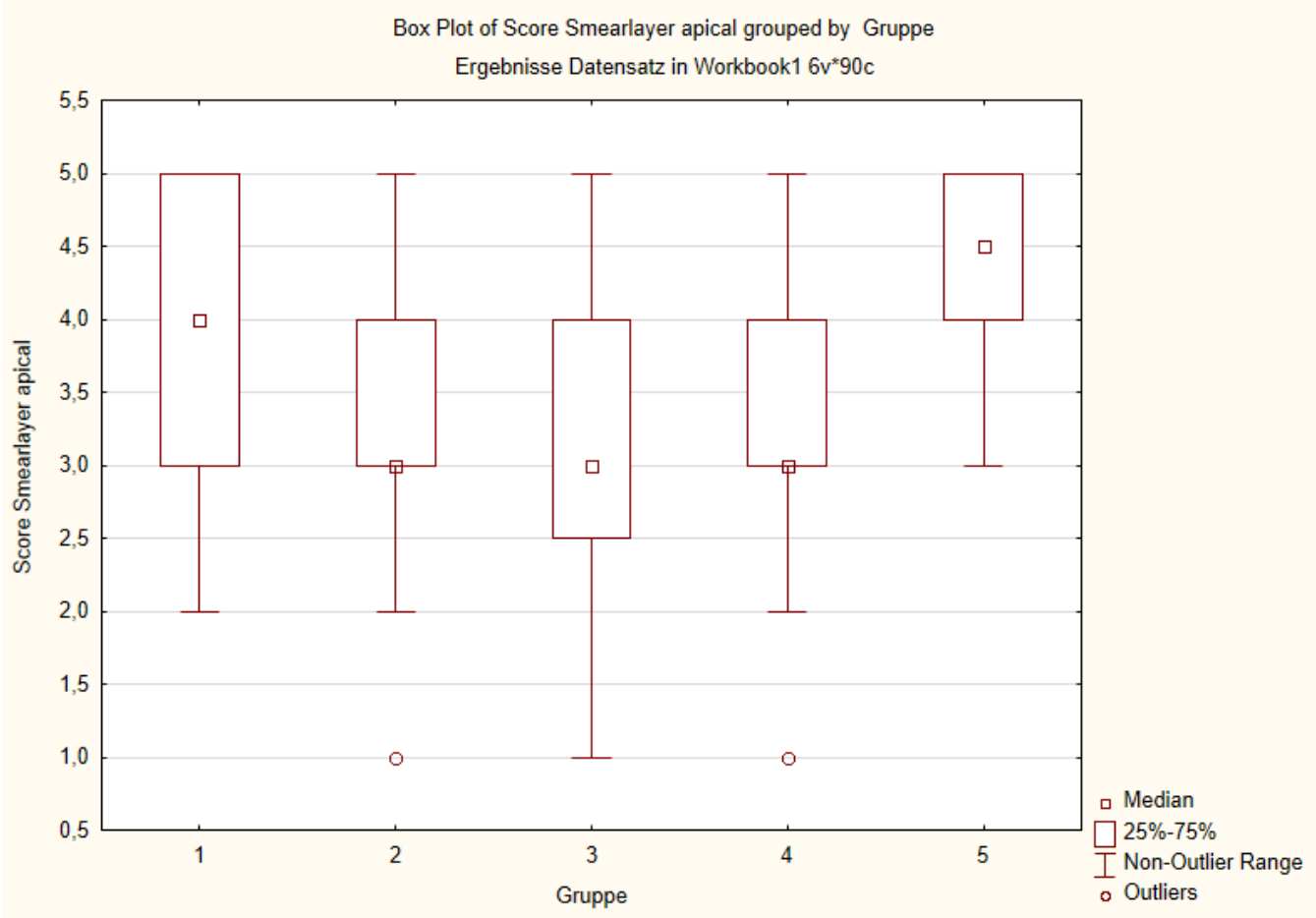

Abbildung 41: Box-Plot der apikalen smear layer-Scores 


\subsubsection{Mann-Whitney-U-Tests}

Vergleich Gruppe 1 (Dynamische Handspülung) und Gruppe 2 (EndoActivator®)

\begin{tabular}{|c|c|c|c|c|c|c|c|c|c|c|}
\hline \multirow[b]{2}{*}{ variable } & \multicolumn{10}{|c|}{ Mann-Whitney U Test (w/ continuity correction) (Ergebnisse Datensatz in Workbook1) By variable Gruppe Marked tests are significant at p<,05000 Gruppe 1 - 2} \\
\hline & \begin{tabular}{|l|} 
Rank Sum \\
Group 1
\end{tabular} & $\begin{array}{c}\text { Rank Sum } \\
\text { Group } 2\end{array}$ & 0 & 2 & p-value & $\mid$\begin{tabular}{c|}
$\mathrm{Z}$ \\
adjusted
\end{tabular} & p-value & \begin{tabular}{||l|} 
Valid N \\
Group 1
\end{tabular} & \begin{tabular}{|l|} 
Valid N \\
Group 2
\end{tabular} & \begin{tabular}{|l|}
$2^{\star} 1$ sided \\
exact $p$
\end{tabular} \\
\hline \begin{tabular}{|l|} 
Score Debris apical \\
\end{tabular} & 487,0000 & 333,0000 & 123.0000 & 2,069332 & 0,038516 & 2,229667 & 0,025770 & & & 0,037519 \\
\hline
\end{tabular}

Tabelle 17: Ergebnisse des Mann-Whitney-U-Tests für Debris apikal (Gruppe 1 gegen Gruppe 2) (Statistica)

Vergleich Gruppe 1 (Dynamische Handspülung) und Gruppe 3 (IrriSafe ${ }^{\mathrm{TM}}$ )

\begin{tabular}{|c|c|c|c|c|c|c|c|c|c|c|}
\hline \multirow[b]{2}{*}{ variable } & \multicolumn{10}{|c|}{ Mann-Whitney U Test (w/ continuity correction) (Ergebnisse Datensatz in Workbook1) By variable Gruppe Marked tests are significant at p<,05000 Gruppe 1 - 3} \\
\hline & \begin{tabular}{|c|} 
Rank Sum \\
Group 1
\end{tabular} & \begin{tabular}{|c|} 
Rank Sum \\
Group 2
\end{tabular} & U & 2 & p-value & \begin{tabular}{|c|}
$\mathrm{Z}$ \\
adjusted
\end{tabular} & p-value & \begin{tabular}{|l|} 
Valid N \\
Group 1
\end{tabular} & \begin{tabular}{|l|} 
Valid N \\
Group 2
\end{tabular} & \begin{tabular}{|l|}
$2 * 1$ sided \\
exact p
\end{tabular} \\
\hline \begin{tabular}{|l|} 
Score Debris apical \\
\end{tabular} & 456,5000 & 363,5000 & 153,5000 & 1,244304 & 0,213389 & 1,337587 & 0,181032 & & 20 & 0,210964 \\
\hline
\end{tabular}

Tabelle 18: Ergebnisse des Mann-Whitney-U-Tests für Debris apikal (Gruppe 1 gegen Gruppe 3) (Statistica)

Vergleich Gruppe 1 (Dynamische Handspülung) und Gruppe 4 (EDDY ${ }^{\circledR}$ )

\begin{tabular}{|c|c|c|c|c|c|c|c|c|c|c|}
\hline \multirow[b]{2}{*}{ variable } & \multicolumn{10}{|c|}{ Mann-Whitney U Test (w/ continuity correction) (Ergebnisse Datensatz in Workbook1) By variable Gruppe Marked tests are significant at $p<, 05000$ Gruppe 1 - 4} \\
\hline & \begin{tabular}{|c|} 
Rank Sum \\
Group 1 \\
\end{tabular} & \begin{tabular}{|c|} 
Rank Sum \\
Group 2
\end{tabular} & (U) & 2 & p-value & $\begin{array}{c}\mathrm{Z} \\
\text { adjusted }\end{array}$ & p-value & \begin{tabular}{|l|} 
Valid N \\
Group 1 \\
\end{tabular} & \begin{tabular}{|l|} 
Valid N \\
Group 2 \\
\end{tabular} & $\begin{array}{l}2^{*} \text { 1sided } \\
\text { exact } p\end{array}$ \\
\hline Score Debris apical & 447,5000 & 372,5000 & 162,5000 & 1,000853 & 0,316898 & 1,086538 & 0,277242 & 2 & & 0,314084 \\
\hline
\end{tabular}

Tabelle 19: Ergebnisse des Mann-Whitney-U-Tests für Debris apikal (Gruppe 1 gegen Gruppe 4) (Statistica)

Vergleich Gruppe 1 (Dynamische Handspülung) und Gruppe 5 (Kontrollgruppe)

\begin{tabular}{|c|c|c|c|c|c|c|c|c|c|c|}
\hline \multirow[b]{2}{*}{ variable } & \multicolumn{10}{|c|}{ Mann-Whitney U Test (w/ continuity correction) (Ergebnisse Datensatz in Workbook1) By variable Gruppe Marked tests are significant at p<,05000 Gruppe 1 - 5} \\
\hline & \begin{tabular}{|l|} 
Rank Sum \\
Group 1 \\
\end{tabular} & \begin{tabular}{|c|} 
Rank Sum \\
Group 2
\end{tabular} & U & 2 & p-value & $\begin{array}{c}\mathrm{Z} \\
\text { adjusted }\end{array}$ & p-value & \begin{tabular}{|l|} 
Valid N \\
Group 1 \\
\end{tabular} & \begin{tabular}{|l|} 
Valid N \\
Group 2
\end{tabular} & $\begin{array}{l}2^{*} 1 \text { sided } \\
\text { exact } p\end{array}$ \\
\hline Score Debris apical & 270,0000 & 195,0000 & 60,00000 & $-1,73777$ & 0,082253 & $-1,81315$ & 0,069809 & & & 0,082209 \\
\hline
\end{tabular}

Tabelle 20: Ergebnisse des Mann-Whitney-U-Tests für Debris apikal (Gruppe 1 gegen Gruppe 5) (Statistica) 
Vergleich Gruppe 2 (EndoActivator®) und Gruppe 3 (IrriSafe ${ }^{\mathrm{TM}}$ )

\begin{tabular}{|c|c|c|c|c|c|c|c|c|c|c|}
\hline \multirow[b]{2}{*}{ variable } & \multicolumn{10}{|c|}{ Mann-Whitney U Test (w/ continuity correction) (Ergebnisse Datensatz in Workbook1) By variable Gruppe Marked tests are significant at p<,05000 Gruppe 2 - 3} \\
\hline & \begin{tabular}{|l|} 
Rank Sum \\
Group 1 \\
\end{tabular} & \begin{tabular}{|l|} 
Rank Sum \\
Group 2 \\
\end{tabular} & & 8 & p-value & \begin{tabular}{|c|}
$Z$ \\
adjusted
\end{tabular} & p-value & \begin{tabular}{|l|} 
Valid N \\
Group 1
\end{tabular} & & \begin{tabular}{|l|}
$2 * 1$ sided \\
exact $p$
\end{tabular} \\
\hline Score Debris apical & 379,5000 & 440,5000 & 169,5000 & $-0,811503$ & 0,417078 & $-0,882196$ & 0,377671 & 2 & & 0,413526 \\
\hline
\end{tabular}

Tabelle 21: Ergebnisse des Mann-Whitney-U-Tests für Debris apikal (Gruppe 2 gegen Gruppe 3) (Statistica)

Vergleich Gruppe 2 (EndoActivator $\left.{ }^{\circledR}\right)$ und Gruppe 4 (EDDY ${ }^{\circledR}$ )

\begin{tabular}{|c|c|c|c|c|c|c|c|c|c|c|}
\hline \multirow[b]{2}{*}{ variable } & \multicolumn{10}{|c|}{ Mann-Whitney U Test (w/ continuity correction) (Ergebnisse Datensatz in Workbook1) By variable Gruppe Marked tests are significant at p<,05000 Gruppe 2 - 4} \\
\hline & \begin{tabular}{|c|} 
Rank Sum \\
Group 1 \\
\end{tabular} & \begin{tabular}{|l|} 
Rank Sum \\
Group 2
\end{tabular} & U & Z & p-value & \begin{tabular}{|c|}
$\mathrm{Z}$ \\
adjusted
\end{tabular} & p-value & \begin{tabular}{|l|} 
Valid N \\
Group 1 \\
\end{tabular} & \begin{tabular}{|l|} 
Valid N \\
Group 2 \\
\end{tabular} & \begin{tabular}{|l|}
$2^{*} 1$ sided \\
exact $p$
\end{tabular} \\
\hline Score Debris apical & 370,0000 & 450,0000 & 160,0000 & $-1,06848$ & 0,285306 & $-1,17028$ & 0,241888 & & 2 & 0,288767 \\
\hline
\end{tabular}

Tabelle 22: Ergebnisse des Mann-Whitney-U-Tests für Debris apikal (Gruppe 2 gegen Gruppe 4) (Statistica)

Vergleich Gruppe 2 (EndoActivator®) und Gruppe 5 (Kontrollgruppe)

\begin{tabular}{|c|c|c|c|c|c|c|c|c|c|c|}
\hline \multirow[b]{2}{*}{ variable } & \multicolumn{10}{|c|}{ Mann-Whitney U Test (w/ continuity correction) (Ergebnisse Datensatz in Workbook1) By variable Gruppe Marked tests are significant at p<,05000 Gruppe 2 - 5} \\
\hline & \begin{tabular}{|l|} 
Rank Sum \\
Group 1 \\
\end{tabular} & \begin{tabular}{|l|} 
Rank Sum \\
Group 2 \\
\end{tabular} & U & Z & $p$-value & $\begin{array}{c}Z \\
\text { adjusted }\end{array}$ & p-value & \begin{tabular}{|l|} 
Valid N \\
Group 1 \\
\end{tabular} & \begin{tabular}{|l|} 
Valid N \\
Group 2 \\
\end{tabular} & $\begin{array}{l}2^{*} 1 \text { sided } \\
\text { exact p }\end{array}$ \\
\hline Score Debris apical & 248,0000 & 217,0000 & 38,00000 & $-2,70564$ & 0,006818 & $-2,83782$ & 0,004542 & & 20 & 0,005287 \\
\hline
\end{tabular}

Tabelle 23: Ergebnisse des Mann-Whitney-U-Tests für Debris apikal (Gruppe 2 gegen Gruppe 5) (Statistica)

Vergleich Gruppe 3 (IrriSafe ${ }^{\mathrm{TM}}$ ) und Gruppe 4 (EDDY ${ }^{\circledR}$ )

\begin{tabular}{|c|c|c|c|c|c|c|c|c|c|c|}
\hline \multirow[b]{2}{*}{ variable } & \multicolumn{10}{|c|}{ Mann-Whitney U Test (w/ continuity correction) (Ergebnisse Datensatz in Workbook1) By variable Gruppe Marked tests are significant at p<,05000 Gruppe 3 - 4} \\
\hline & \begin{tabular}{|c|} 
Rank Sum \\
Group 1 \\
\end{tabular} & \begin{tabular}{|l|} 
Rank Sum \\
Group 2 \\
\end{tabular} & (1) & 8 & p-value & \begin{tabular}{|c|}
$Z$ \\
adjusted \\
\end{tabular} & p-value & \begin{tabular}{|l|} 
Valid N \\
Group 1 \\
\end{tabular} & \begin{tabular}{|l|} 
Valid N \\
Group 2 \\
\end{tabular} & \begin{tabular}{|l|}
$2 * 1$ sided \\
exact $p$ \\
\end{tabular} \\
\hline Score Debris apical & 401,0000 & 419,0000 & 191,0000 & $-0,229926$ & 0,818150 & $-0,250651$ & 0,802084 & 20 & 20 & 0,820148 \\
\hline
\end{tabular}

Tabelle 24: Ergebnisse des Mann-Whitney-U-Tests für Debris apikal (Gruppe 3 gegen Gruppe 4) (Statistica) 
Vergleich Gruppe 3 (IrriSafe $^{\mathrm{TM}}$ ) und Gruppe 5 (Kontrollgruppe)

\begin{tabular}{|c|c|c|c|c|c|c|c|c|c|c|c|}
\hline \multirow[b]{2}{*}{ variable } & \multicolumn{11}{|c|}{ Mann-Whitney U Test (w/ continuity correction) (Ergebnisse Datensatz in Workbooki) By variable Gruppe Marked tests are significant at p<,05000 Gruppe 3-5 } \\
\hline & \begin{tabular}{|c|} 
Rank Sum \\
Group 1 \\
\end{tabular} & \begin{tabular}{|l|} 
Rank Sum \\
Group 2
\end{tabular} & 0 & $z$ & & p-value & \begin{tabular}{|c|}
$Z$ \\
adjusted
\end{tabular} & p-value & \begin{tabular}{|l|}
$\begin{array}{l}\text { Valid N } \\
\text { Group 1 }\end{array}$ \\
\end{tabular} & \begin{tabular}{|l|} 
Valid \\
Group 2
\end{tabular} & $\mid$\begin{tabular}{l|}
$2^{*} 1$ sided \\
exact $p$
\end{tabular} \\
\hline Score Debris apical & 258.0000 & & & 48,00000 & -2.26570 & 0.023471 & -2.37002 & 0,017788 & & 20 & 0,021557 \\
\hline
\end{tabular}

Tabelle 25: Ergebnisse des Mann-Whitney-U-Tests für Debris apikal (Gruppe 3 gegen Gruppe 5) (Statistica)

Vergleich Gruppe 4 (EDDY ${ }^{\circledR}$ ) und Gruppe 5 (Kontrollgruppe)

\begin{tabular}{|c|c|c|c|c|c|c|c|c|c|c|c|}
\hline \multirow[b]{2}{*}{ variable } & \multicolumn{11}{|c|}{ Mann-Whitney U Test (w/ continuity correction) (Ergebnisse Datensatz in Workbook1) By variable Gruppe Marked tests are significant atp <,05000 Gruppe 4-5 } \\
\hline & \begin{tabular}{|l|} 
Rank Sum \\
Group 1
\end{tabular} & \begin{tabular}{|l|} 
Rank Sum \\
Group 2
\end{tabular} & U & $z$ & & p-value & \begin{tabular}{|c|}
$Z$ \\
adjusted
\end{tabular} & p-value & \begin{tabular}{|l|} 
Valid N \\
Group 1
\end{tabular} & \begin{tabular}{|l|} 
Valid N \\
Group 2 \\
\end{tabular} & $\mid \begin{array}{l}2^{*} 1 \text { sided } \\
\text { exact p p }\end{array}$ \\
\hline Score Debris apical & 260,5000 & 204, & & 50,50000 & $-2,15571$ & 0,031107 & $-2,27106$ & 0,023144 & & 20 & 0,027603 \\
\hline
\end{tabular}

Tabelle 26: Ergebnisse des Mann-Whitney-U-Tests für Debris apikal (Gruppe 4 gegen Gruppe 5) (Statistica)

Vergleich Gruppe 1 (Dynamische Handspülung) und Gruppe 2 (EndoActivator®)

\begin{tabular}{|c|c|c|c|c|c|c|c|c|c|c|}
\hline \multirow[b]{2}{*}{ variable } & \multicolumn{10}{|c|}{ Mann-Whitney U Test (w/ continuity correction) (Ergebnisse Datensatz in Workbook1) By variable Gruppe Marked tests are significant at p<,05000 Gruppe 1 - 2} \\
\hline & \begin{tabular}{|c|} 
Rank Sum \\
Group 1 \\
\end{tabular} & \begin{tabular}{|c|} 
Rank Sum \\
Group 2 \\
\end{tabular} & & 2 & p-value & $\begin{array}{c}Z \\
\text { adjusted }\end{array}$ & p-value & \begin{tabular}{|l|} 
Valid N \\
Group 1 \\
\end{tabular} & \begin{tabular}{|l|} 
Valid N \\
Group 2 \\
\end{tabular} & \begin{tabular}{|l|}
$2 * 1$ sided \\
exactp
\end{tabular} \\
\hline Score Smearlayer apical & 476,0000 & 344,0000 & 134,0000 & 1,771781 & 0,076432 & 1,863546 & 0,062386 & 20 & 2 & 0,076266 \\
\hline
\end{tabular}

Tabelle 27: Ergebnisse des Mann-Whitney-U-Tests für smear layer apikal (Gruppe 1 gegen Gruppe 2) (Statistica)

Vergleich Gruppe 1 (Dynamische Handspülung) und Gruppe 3 (IrriSafe ${ }^{\mathrm{TM}}$ )

\begin{tabular}{|c|c|c|c|c|c|c|c|c|c|c|}
\hline \multirow[b]{2}{*}{ variable } & \multicolumn{10}{|c|}{ Mann-Whitney U Test (w/ continuity correction) (Ergebnisse Datensatz in Workbook1) By variable Gruppe Marked tests are significant at p<,05000 Gruppe 1 - 3} \\
\hline & \begin{tabular}{|c|} 
Rank Sum \\
Group 1 \\
\end{tabular} & \begin{tabular}{|c|} 
Rank Sum \\
Group 2
\end{tabular} & (1) & 2 & p-value & $\begin{array}{c}Z \\
\text { adjusted }\end{array}$ & p-value & \begin{tabular}{|l|} 
Valid N \\
Group 1 \\
\end{tabular} & \begin{tabular}{|l|} 
Valid N \\
Group 2 \\
\end{tabular} & \begin{tabular}{|l|}
$2^{*} 11$ sided \\
exact p
\end{tabular} \\
\hline Score Smearlayer apical & 496,5000 & 323,5000 & 113,5000 & 2,326308 & 0,020003 & 2,429459 & 0,015122 & & 20 & 0,018094 \\
\hline
\end{tabular}

Tabelle 28: Ergebnisse des Mann-Whitney-U-Tests für smear layer apikal (Gruppe 1 gegen Gruppe 3) (Statistica) 
Vergleich Gruppe 1 (Dynamische Handspülung) und Gruppe 4 (EDDY ${ }^{\circledR}$ )

\begin{tabular}{|c|c|c|c|c|c|c|c|c|c|c|c|}
\hline \multirow[b]{2}{*}{ variable } & \multicolumn{11}{|c|}{ Mann-Whitney U Test (w/ continuity correction) (Ergebnisse Datensatz in Workbook1) By variable Gruppe Marked tests are significant at p<,05000 Gruppe 1 - 4} \\
\hline & \begin{tabular}{|c|} 
Rank Sum \\
Group 1 \\
\end{tabular} & \begin{tabular}{|c|} 
Rank Sum \\
Group 2 \\
\end{tabular} & U & 8 & p-value & \begin{tabular}{|c|}
$Z$ \\
adjusted
\end{tabular} & p-value & \begin{tabular}{|l|} 
Valid N \\
Group 1 \\
\end{tabular} & \begin{tabular}{|l|} 
Valid N \\
Group 2 \\
\end{tabular} & & $\begin{array}{l}2^{*} 1 \text { sided } \\
\text { exact p }\end{array}$ \\
\hline Score Smearlayer apical & 479,0000 & 341,0000 & 131,0000 & 1,852931 & 0,063893 & 1,972688 & 0,048532 & 2 & & 20 & 0,063432 \\
\hline
\end{tabular}

Tabelle 29: Ergebnisse des Mann-Whitney-U-Tests für smear layer apikal (Gruppe 1 gegen Gruppe 4) (Statistica)

Vergleich Gruppe 1 (Dynamische Handspülung) und Gruppe 5 (Kontrollgruppe)

\begin{tabular}{|c|c|c|c|c|c|c|c|c|c|c|}
\hline \multirow[b]{2}{*}{ variable } & \multicolumn{10}{|c|}{ Mann-Whitney U Test (w/ continuity correction) (Ergebnisse Datensatz in Workbook1) By variable Gruppe Marked tests are significant at p<,05000 Gruppe 1 - 5} \\
\hline & \begin{tabular}{|c|} 
Rank Sum \\
Group 1 \\
\end{tabular} & \begin{tabular}{|c|} 
Rank Sum \\
Group 2
\end{tabular} & U & 8 & p-value & \begin{tabular}{|c|}
$z$ \\
adjusted
\end{tabular} & p-value & \begin{tabular}{|l|} 
Valid N \\
Group 1 \\
\end{tabular} & \begin{tabular}{|l|} 
Valid N \\
Group 2 \\
\end{tabular} & $\begin{array}{l}2 * 1 \text { sided } \\
\text { exact } p\end{array}$ \\
\hline Score Smearlayer apical & 279,0000 & 186,0000 & 69,00000 & $-1,34182$ & 0,179655 & $-1,42260$ & 0,154852 & & 0 & 0,182884 \\
\hline
\end{tabular}

Tabelle 30: Ergebnisse des Mann-Whitney-U-Tests für smear layer apikal (Gruppe 1 gegen Gruppe 5) (Statistica)

Vergleich Gruppe 2 (EndoActivator®) und Gruppe 3 (IrriSafe ${ }^{\mathrm{TM}}$ )

\begin{tabular}{|c|c|c|c|c|c|c|c|c|c|c|}
\hline \multirow[b]{2}{*}{ variable } & \multicolumn{10}{|c|}{ Mann-Whitney U Test (w/ continuity correction) (Ergebnisse Datensatz in Workbook1) By variable Gruppe Marked tests are significant at p<,05000 Gruppe 2 - 3} \\
\hline & \begin{tabular}{|c|} 
Rank Sum \\
Group 1 \\
\end{tabular} & \begin{tabular}{|l|} 
Rank Sum \\
Group 2 \\
\end{tabular} & U & Z & p-value & \begin{tabular}{c|}
$Z$ \\
adjusted
\end{tabular} & p-value & \begin{tabular}{|l|l|} 
Valid N \\
Group 1 \\
\end{tabular} & \begin{tabular}{|l|} 
Valid N \\
Group 2 \\
\end{tabular} & \begin{tabular}{|l|}
$2^{*} 1$ sided \\
exactp
\end{tabular} \\
\hline Score Smearlayer apical & 430,5000 & 389,5000 & 179,5000 & 0,541002 & 0,588507 & 0,573322 & 0,566427 & & 20 & 0,583114 \\
\hline
\end{tabular}

Tabelle 31: Ergebnisse des Mann-Whitney-U-Tests für smear layer apikal (Gruppe 2 gegen Gruppe 3) (Statistica)

Vergleich Gruppe 2 (EndoActivator $\left.{ }^{\circledR}\right)$ und Gruppe 4 (EDDY ${ }^{\circledR}$ )

\begin{tabular}{|c|c|c|c|c|c|c|c|c|c|c|}
\hline \multirow[b]{2}{*}{ variable } & \multicolumn{10}{|c|}{ Mann-Whitney U Test (w/ continuity correction) (Ergebnisse Datensatz in Workbook1) By variable Gruppe Marked tests are significant at p<,05000 Gruppe 2 - 4} \\
\hline & \begin{tabular}{|c|} 
Rank Sum \\
Group 1 \\
\end{tabular} & \begin{tabular}{|l|} 
Rank Sum \\
Group 2 \\
\end{tabular} & 4 & 2 & p-value & \begin{tabular}{|c|}
$Z$ \\
adjusted \\
\end{tabular} & p-value & \begin{tabular}{|l|} 
Valid N \\
Group 1 \\
\end{tabular} & \begin{tabular}{|l|} 
Valid N \\
Group 2 \\
\end{tabular} & \begin{tabular}{|l|}
$\begin{array}{l}2^{*} 1 \text { sided } \\
\text { exact p }\end{array}$ \\
\end{tabular} \\
\hline Score Smearlayer apical & 411,0000 & 409,0000 & 199,0000 & 0,01353 & 0,989209 & 0,01489 & 0,988118 & 20 & 2 & 0,989332 \\
\hline
\end{tabular}

Tabelle 32: Ergebnisse des Mann-Whitney-U-Tests für smear layer apikal (Gruppe 2 gegen Gruppe 4) (Statistica) 
Vergleich Gruppe 2 (EndoActivator®) und Gruppe 5 (Kontrollgruppe)

\begin{tabular}{|c|c|c|c|c|c|c|c|c|c|c|}
\hline \multirow{2}{*}{ variable } & \multicolumn{10}{|c|}{ Mann-Whitney U Test (w/ continuity correction) (Ergebnisse Datensatz in Workbook1) By variable Gruppe Marked tests are significant at p<,05000 Gruppe 2 - 5} \\
\hline & \begin{tabular}{|l|} 
Rank Sum \\
Group 1
\end{tabular} & \begin{tabular}{|c|} 
Rank Sum \\
Group 2
\end{tabular} & 0 & 8 & p-value & $\begin{array}{c}Z \\
\text { adjusted }\end{array}$ & p-value & \begin{tabular}{|l|} 
Valid N \\
Group 1
\end{tabular} & & $\begin{array}{l}2 * 1 \text { sided } \\
\text { exact p }\end{array}$ \\
\hline Score Smearlayer apical & 249,5000 & 215,5000 & 39,50000 & $-2,63965$ & 0,008300 & $-2,76186$ & 0,005748 & & 0 & 0,006169 \\
\hline
\end{tabular}

Tabelle 33: Ergebnisse des Mann-Whitney-U-Tests für smear layer apikal (Gruppe 2 gegen Gruppe 4) (Statistica)

Vergleich Gruppe 3 (IrriSafe ${ }^{\mathrm{TM}}$ ) und Gruppe 4 (EDDY $\left.{ }^{\circledR}\right)$

\begin{tabular}{|c|c|c|c|c|c|c|c|c|c|c|}
\hline \multirow[b]{2}{*}{ variable } & \multicolumn{10}{|c|}{ Mann-Whitney U Test (w/ continuity correction) (Ergebnisse Datensatz in Workbook1) By variable Gruppe Marked tests are significant at p<,05000 Gruppe 3 - 4} \\
\hline & \begin{tabular}{|c|} 
Rank Sum \\
Group 1 \\
\end{tabular} & \begin{tabular}{|l|} 
Rank Sum \\
Group 2 \\
\end{tabular} & (U) & 2 & p-value & \begin{tabular}{|c|}
$Z$ \\
adjusted
\end{tabular} & p-value & \begin{tabular}{|l|} 
Valid N \\
Group 1 \\
\end{tabular} & \begin{tabular}{|l|} 
Valid N \\
Group 2 \\
\end{tabular} & \begin{tabular}{|l|}
$2^{*} 1$ sided \\
exact p
\end{tabular} \\
\hline Score Smearlayer apical & 391,0000 & 429,0000 & 181,0000 & $-0,500427$ & 0,616775 & $-0,539083$ & 0,589830 & 20 & 20 & 0,620465 \\
\hline
\end{tabular}

Tabelle 34: Ergebnisse des Mann-Whitney-U-Tests für smear layer apikal (Gruppe 3 gegen Gruppe 4) (Statistica)

Vergleich Gruppe 3 (IrriSafe $^{\mathrm{TM}}$ ) und Gruppe 5 (Kontrollgruppe)

\begin{tabular}{|c|c|c|c|c|c|c|c|c|c|c|c|}
\hline \multirow[b]{2}{*}{ variable } & \multicolumn{11}{|c|}{ Mann-Whitney U Test (w/ continuity correction) (Ergebnisse Datensatz in Workbook1) By variable Gruppe Marked tests are significant at p<,05000 Gruppe 3 - 5} \\
\hline & \begin{tabular}{|c|} 
Rank Sum \\
Group 1 \\
\end{tabular} & \begin{tabular}{|c|} 
Rank Sum \\
Group 2 \\
\end{tabular} & 0 & & 8 & p-value & $\begin{array}{c}Z \\
\text { adjusted }\end{array}$ & p-value & \begin{tabular}{|l|} 
Valid N \\
Group 1 \\
\end{tabular} & \begin{tabular}{|l|} 
Valid N \\
Group 2 \\
\end{tabular} & $\begin{array}{l}2^{*} 1 \text { sided } \\
\text { exactp }\end{array}$ \\
\hline Score Smearlayer apical & 239,5000 & & & 29,50000 & $-3,07959$ & 0,002073 & $-3,19735$ & 0,001387 & & 20 & 0,001116 \\
\hline
\end{tabular}

Tabelle 35: Ergebnisse des Mann-Whitney-U-Tests für smear layer apikal (Gruppe 3 gegen Gruppe 5) (Statistica)

Vergleich Gruppe 4 (EDDY ${ }^{\circledR}$ ) und Gruppe 5 (Kontrollgruppe)

\begin{tabular}{|c|c|c|c|c|c|c|c|c|c|c|}
\hline \multirow[b]{2}{*}{ variable } & \multicolumn{10}{|c|}{ Mann-Whitney U Test (w/ continuity correction) (Ergebnisse Datensatz in Workbook1) By variable Gruppe Marked tests are significant at p<,05000 Gruppe 4 - 5} \\
\hline & \begin{tabular}{|c|} 
Rank Sum \\
Group 1 \\
\end{tabular} & \begin{tabular}{|c|} 
Rank Sum \\
Group 2
\end{tabular} & 4 & 2 & p-value & $\begin{array}{c}Z \\
\text { adjusted }\end{array}$ & p-value & \begin{tabular}{|l|} 
Valid N \\
Group 1 \\
\end{tabular} & \begin{tabular}{|l|} 
Valid N \\
Group 2 \\
\end{tabular} & \begin{tabular}{|l|}
$2 * 1$ sided \\
exact $p$
\end{tabular} \\
\hline Score Smearlayer apical & 247,5000 & 217,5000 & 37,50000 & $-2,72764$ & 0,006379 & $-2,89475$ & 0,003795 & 2 & 20 & 0,004515 \\
\hline
\end{tabular}

Tabelle 36: Ergebnisse des Mann-Whitney-U-Tests für smear layer apikal (Gruppe 4 gegen Gruppe 5) (Statistica) 


\subsection{Herstellerverzeichnis/Materialliste}

\begin{tabular}{|l|l|}
\hline $\begin{array}{l}\text { Aluminiumnieten } \\
\text { DIN 661, 3,0 mm x 12,0 mm }\end{array}$ & $\begin{array}{l}\text { Gesipa, Mörfelden-Walldorf, } \\
\text { Deutschland }\end{array}$ \\
\hline $\begin{array}{l}\text { BA Optima 10 LED } \\
\text { Polymerisationslampe }\end{array}$ & $\begin{array}{l}\text { B.A. International, Northhampton, } \\
\text { England }\end{array}$ \\
\hline BioRaCe ${ }^{\text {TM_System }}$ & $\begin{array}{l}\text { FKG Dentaire, La-Chaux des-Fonds, } \\
\text { Schweiz }\end{array}$ \\
\hline CanoScan 9000F Durchlichtscanner & Canon, Tokio, Japan \\
\hline $\begin{array}{l}\text { Diamantierte Trennscheibe (Komet } \\
\text { Dental, Lemgo) }\end{array}$ & Komet Dental, Lemgo, Deutschland \\
\hline Diamantierte zylindrische Schleifer & Komet Dental, Lemgo, Deutschland \\
\hline EDDY® Spülspitze & VDW, München, Deutschland \\
\hline EndoActivator® & $\begin{array}{l}\text { Dentsply Sirona, York, Pennsylvania, } \\
\text { USA }\end{array}$ \\
\hline FinoDisc Diamantierte Trennscheibe & Fino, Bad Bocklet, Deutschland \\
\hline Geodreieck & Pelikan AG, Berlin, Deutschland \\
\hline Guttapercha ISO Größe 35 & VDW, München, Deutschland \\
\hline Hedströmfeilen ISO Größen 10 und 15 & VDW, München, Deutschland \\
\hline Heidemannspatel & Heraeus, Hanau, Deutschland \\
\hline $\begin{array}{l}\text { ImageJ 1.50i } \\
\text { Bildbearbeitungssoftware }\end{array}$ & $\begin{array}{l}\text { Wayne Rasband, National Institutes } \\
\text { of Health, Rockville, Maryland, USA }\end{array}$ \\
\hline IrriSafe ${ }^{\text {TM }}$ Spülspitze Größe 20/21 & Acteon Group, Norwich, England \\
\hline Kunststoffspritze 5 ml & B. Braun, Melsungen, Deutschland \\
\hline Leica EM ACE200 Feinvakuum- & $\begin{array}{l}\text { Leica Microsystems, Wetzlar, } \\
\text { Deutschland }\end{array}$ \\
\hline Lichthärtendes Komposit Venus & Heraeus, Hanau, Deutschland \\
\hline
\end{tabular}




\begin{tabular}{|c|c|}
\hline Microsoft Office & $\begin{array}{l}\text { Microsoft Corporation, Redmond, } \\
\text { Washington, USA }\end{array}$ \\
\hline Natriumhypochlorit 3\% & $\begin{array}{l}\text { Apotheke des Universitätsklinikums } \\
\text { Göttingen, Deutschland }\end{array}$ \\
\hline Paladur Prothesenkunststoff & Heraeus, Hanau, Deutschland \\
\hline Peripheriewachs, blau & $\begin{array}{l}\text { American Dental Systems, } \\
\text { Vaterstetten, Deutschland }\end{array}$ \\
\hline $\begin{array}{l}\text { Photoshop CC } 2015 \\
\text { Bildbearbeitungssoftware }\end{array}$ & $\begin{array}{l}\text { Adobe Incorporated, San José, } \\
\text { Kalifornien, USA }\end{array}$ \\
\hline Proxeo ZA 55LM Airscaler & W\&H, Bürmoos, Salzburg, Österreich \\
\hline Reamer ISO Größe 10 & Orbis Dental, Münster, Deutschland \\
\hline Reamer ISO Größen 10 und 15 & VDW, München, Deutschland \\
\hline Röntgenfilm Kodak Insight & Kodak, Rochester, New York, USA \\
\hline Röntgengerät Heliodent DS & Sirona, Bensheim, Deutschland \\
\hline Statistica Statistiksoftware & $\begin{array}{l}\text { StatSoft Europe GmbH, Hamburg, } \\
\text { Deutschland }\end{array}$ \\
\hline Vaseline, weiß & $\begin{array}{l}\text { Bombastus-Werke AG, Freital, } \\
\text { Deutschland }\end{array}$ \\
\hline VDW Ultra UItraschall-Mikromotor & VDW, München, Deutschland \\
\hline $\begin{array}{l}\text { VMK Endoneedle nach Dr. J. Buquet } \\
\text { Spülkanüle }\end{array}$ & Vedefar NV, Dilbeek, Belgien \\
\hline Wachsmesser & Heraeus, Hanau, Deutschland \\
\hline $\begin{array}{l}\text { Zeiss Ultra Plus Feldemissions- } \\
\text { Rasterelektronenmikroskops }\end{array}$ & $\begin{array}{l}\text { Carl Zeiss AG, Oberkochen, } \\
\text { Deutschland }\end{array}$ \\
\hline Zirkel & Pelikan AG, Berlin, Deutschland \\
\hline
\end{tabular}

Tabelle 37: Herstellerverzeichnis und Materialliste 


\section{$9 \quad$ Literaturverzeichnis}

Ahmad M, Pitt Ford TR, Crum LA (1987): Ultrasonic debridement of root canals: Acoustic streaming and its possible role. J Endod 13, 490-499

Al-Ali M, Sathorn C, Parashos P (2012): Root canal debridement efficacy of different final irrigation protocols. Int Endod J 논, 898-906

Alam MS, Bashar AK, Begumr JA, Kinoshita JI (2006): A study on FlexMaster: a Ni-Ti rotary engine driven system for root canal preparation. Mymensingh Med J MMJ $\underline{15}, 135-141$

Ari H, Erdemir A (2005): Effects of endodontic irrigation solutions on mineral content of root canal dentin using ICP-AES technique. J Endod $\underline{31}$, 187-189 Ari H, Erdemir A, Belli S (2004): Evaluation of the effect of endodontic irrigation solutions on the microhardness and the roughness of root canal dentin. J Endod 30, 792-795

Arslan D, Guneser MB, Dincer AN, Kustarci A, Er K, Siso SH (2016): Comparison of Smear Layer Removal Ability of QMix with Different Activation Techniques. J Endod 42, 1279-1285

Arslan H, Akcay M, Capar ID, Ertas H, Ok E, Uysal B (2014): Efficacy of needle irrigation, EndoActivator, and photon-initiated photoacoustic streaming technique on removal of double and triple antibiotic pastes. $J$ Endod 40, 1439-1442

Aslantas EE, Buzoglu HD, Altundasar E, Serper A (2014): Effect of EDTA, sodium hypochlorite, and chlorhexidine gluconate with or without surface modifiers on dentin microhardness. J Endod $\underline{40}, 876-879$

Ballal NV, Jain I, Tay FR (2016): Evaluation of the smear layer removal and decalcification effect of QMix, maleic acid and EDTA on root canal dentine. J Dent $\underline{51}, 62-68$

Barrieshi-Nusair KM (2002): Gutta-percha retreatment: effectiveness of nickel-titanium rotary instruments versus stainless steel hand files. J Endod 
$\underline{28}, 454-456$

Baumgartner JC, Mader CL (1987): A scanning electron microscopic evaluation of four root canal irrigation regimens. J Endod $\underline{13}, 147-157$

Behrend GD, Cutler CW, Gutmann JL (1996): An in-vitro study of smear layer removal and microbial leakage along root-canal fillings. Int Endod J $\underline{29}$, 99107

Bramante CM, Berbert A, Borges RP (1987): A methodology for evaluation of root canal instrumentation. J Endod 13, 243-245

Brannström M, Nordenvall KJ, Glantz P-O (1980): The Effect of EDTAcontaining Surface-active Solutions on the Morphology of Prepared Dentin: An in vivo Study. J Dent Res $\underline{59}, 1127-1131$

Bueno CE da S, Delboni MG, de Araújo RA, Carrara HJ, Cunha RS (2006): Effectiveness of rotary and hand files in gutta-percha and sealer removal using chloroform or chlorhexidine gel. Braz Dent J $\underline{17}, 139-143$

Byström A, Sunvqvist G (1985): The antibacterial action of sodium hypochlorite and EDTA in 60 cases of endodontic therapy. Int Endod J $\underline{18}$, $35-40$

Calt S, Serper A (2002): Time-dependent effects of EDTA on dentin structures. J Endod 28, $17-19$

Campos JM, del Rio C (1990): Comparison of mechanical and standard hand instrumentation techniques in curved root canals. J Endod 16, 230-234

Çapar ID, Ari Aydinbelge H (2014): Effectiveness of various irrigation activation protocols and the self-adjusting file system on smear layer and debris removal. Scanning $\underline{36}, 640-647$

Caron G, Nham K, Bronnec F, Machtou P (2010): Effectiveness of different final irrigant activation protocols on smear layer removal in curved canals. $J$ Endod $\underline{36}, 1361-1366$

Castelo-Baz P, Varela-Patiño P, Cantatore G, Domínguez-Perez A, RuízPiñón M, Miguéns-Vila R, Martín-Biedma B (2016): In vitro comparison of passive and continuous ultrasonic irrigation in curved root canals. J Clin Exp Dent $\underline{8}$, e437-e441 
Chailertvanitkul P, Saunders WP, MacKenzie D (1996): The effect of smear layer on microbial coronal leakage of gutta-percha root fillings. Int Endod $\mathrm{J}$ $\underline{29}, 242-248$

Chang YC, Huang FM, Tai KW, Chou MY (2001): The effect of sodium hypochlorite and chlorhexidine on cultured human periodontal ligament cells. Oral Surg Oral Med Oral Pathol Oral Radiol Endod 92, 446-450

Chen S, Liu J, Dong G, Peng B, Yang P, Chen Z, Yang F, Guo D (2016): Comparison between ultrasonic irrigation and syringe irrigation in clinical and laboratory studies. J Oral Sci $\underline{58}, 373-378$

Chow TW (1983): Mechanical effectiveness of root canal irrigation. J Endod $\underline{9}, 475-479$

Clarkson R, Moule A, Podlich H, Kellaway R, Macfarlane R, Lewis D, Rowell $J$ (2006): Dissolution of porcine incisor pulps in sodium hypochlorite solutions of varying compositions and concentrations. Aust Dent J $\underline{51}, 245-251$

Conde AJ, Estevez R, Loroño G, Valencia de Pablo Ó, Rossi-Fedele G, Cisneros R (2016): Effect of sonic and ultrasonic activation on organic tissue dissolution from simulated grooves in root canals using sodium hypochlorite and EDTA. Int Endod J 무, 976-982

Cruz-Filho AM, Sousa-Neto MD, Savioli RN, Silva RG, Vansan LP, Pécora JD (2011): Effect of chelating solutions on the microhardness of root canal lumen dentin. J Endod $\underline{37}, 358-362$

Da Silva LAB, Leonardo MR, Assed S, Tanomaru Filho M (2004): Histological study of the effect of some irrigating solutions on bacterial endotoxin in dogs. Braz Dent J $\underline{15}, 109-114$

De Castro FPL, Pinheiro SL, Duarte MAH, Duque JA, Fernandes SL, Anchieta RB, da Silveira Bueno CE (2016): Effect of time and ultrasonic activation on ethylenediaminetetraacetic acid on smear layer removal of the root canal. Microsc Res Tech $\underline{79}$, 1062-1068

Del Carpio-Perochena A, Monteiro Bramante C, Hungaro Duarte $M$, Bombarda de Andrade F, Zardin Graeff M, Marciano da Silva M, Cavalini Cavenago B, Lucas Fernandes S (2015): Effect of Temperature, 
Concentration and Contact Time of Sodium Hypochlorite on the Treatment and Revitalization of Oral Biofilms. J Dent Res Dent Clin Dent Prospects $\underline{9}$, $209-215$

DGZMK/DGZ: Leitlinie zur Wurzelkanalspülung 2006 Version 1.0, 2006. Gemeinsame Stellungnahme der Deutschen Gesellschaft für Zahn-, Mundund Kieferheilkunde (DGZMK) und der Deutschen Gesellschaft für Zahnerhaltung (DGZ). [http://www.dgzmk.de/zahnaerzte/wissenschaftforschung/leitlinien/ details/document/wurzelkanalspuelung.html] abgerufen am: 05.11.2017

Duque JA, Duarte MAH, Canali LCF, Zancan RF, Vivan RR, Bernardes RA, Bramante CM (2016): Comparative Effectiveness of New Mechanical Irrigant Agitating Devices for Debris Removal from the Canal and Isthmus of Mesial Roots of Mandibular Molars. J Endod $\underline{43}$, 326-331

Eick JD, Wilko RA, Anderson CH, Sorensen SE (1970): Scanning Electron Microscopy of Cut Tooth Surfaces and Identification of Debris by Use of the Electron Microprobe. J Dent Res 49, 1359-1368

Elnaghy AM, Mandorah A, Elsaka SE (2016): Effectiveness of XP-endo Finisher, EndoActivator, and File agitation on debris and smear layer removal in curved root canals: a comparative study. Odontology $\underline{105}, 178-183$

Europäische Gesellschaft für Endodontologie (2006): Qualitätsrichtlinien endodontischer Behandlung. Endodontie 15, 387-401.

Frough-Reyhani M, Ghasemi N, Soroush-Barhaghi M, Amini M, Gholizadeh Y (2016): Antimicrobial efficacy of different concentration of sodium hypochlorite on the biofilm of Enterococcus faecalis at different stages of development. J Clin Exp Dent $\underline{8}$, 480-484

Garcia AJA, Kuga MC, Palma-Dibb RG, Só MVR, Matsumoto MA, Faria G, Keine KC (2013): Effect of sodium hypochlorite under several formulations on root canal dentin microhardness. J Investig Clin Dent 4 , 229-232

Gençoğlu N, Samani S, Günday M (1993a): Dentinal wall adaptation of thermoplasticized gutta-percha in the absence or presence of smear layer: a scanning electron microscopic study. J Endod 19, 558-562 
Gençoğlu N, Samani S, Günday M (1993b): Evaluation of sealing properties of Thermafil and Ultrafil techniques in the absence or presence of smear layer. J Endod 19, 599-603

Gergi R, Sabbagh C (2007): Effectiveness of two nickel-titanium rotary instruments and a hand file for removing gutta-percha in severely curved root canals during retreatment: an ex vivo study. Int Endod J $\underline{40}, 532-537$

Giardino L, Mohammadi Z, Beltrami R, Poggio C, Estrela C, Generali L (2016): Influence of Temperature on the Antibacterial Activity of Sodium Hypochlorite. Braz Dent J 27, 32-36

Goldberg F, Spielberg C (1982): The effect of EDTAC and the variation of its working time analyzed with scanning electron microscopy. Oral Surg Oral Med Oral Pathol $\underline{53}, 74-77$

Goldberg F, Massone JE, Spielberg C (1986): Effect of irrigation solutions on the filling of lateral root canals. Endod Dent Traumatol 2, 65-66

Gomes BPFA, Ferraz CCR, Vianna ME, Berber VB, Teixeira FB, Souza-Filho FJ (2001): In vitro antimicrobial activity of several concentrations of sodium hypochlorite and chlorhexidine gluconate in the elimination of Enterococcus faecalis. Int Endod J $\underline{34}$, 424-428

Grawehr M, Sener B, Waltimo T, Zehnder M (2003): Interactions of ethylenediamine tetraacetic acid with sodium hypochlorite in aqueous solutions. Int Endod J $\underline{36}, 411-417$

Groves Cooke H, Grower MF, del Rio C (1976): Effects of instrumentation with a chelating agent on the periapical seal of obturated root canals. J Endod 2, 312-314

Gulsahi K, Tirali RE, Cehreli SB, Karahan ZC, Uzunoglu E, Sabuncuoglu B (2014): The effect of temperature and contact time of sodium hypochlorite on human roots infected with Enterococcus faecalis and Candida albicans. Odontology $\underline{102}, 36-41$

Guo X, Miao H, Li L, Zhang S, Zhou D, Lu Y, Wu L (2014): Efficacy of four different irrigation techniques combined with $60^{\circ} \mathrm{C} 3 \%$ sodium hypochlorite and $17 \%$ EDTA in smear layer removal. BMC Oral Health 14, 114-131 
Gutiérrez JH, Jofré A, Villena F (1990): Scanning electron microscope study on the action of endodontic irrigants on bacteria invading the dentinal tubules. Oral Surg Oral Med Oral Pathol $\underline{69}, 491-501$

Haapasalo M, Endal U, Zandi H, Coil JM (2005): Eradication of endodontic infection by instrumentation and irrigation solutions. Endod Top $\underline{10}$, 77-102

Hergt A, Reus A, Hülsmann M (2017): Removal of calcium hydroxide paste from root canals using four different irrigation techniques. Int Endod J 2017, 50: special issue No. $1, \mathrm{R} 034$

Hsieh YD, Gau CH, Kung Wu SF, Shen EC, Hsu PW, Fu E (2007): Dynamic recording of irrigating fluid distribution in root canals using thermal image analysis. Int Endod J 느, 11-17

Hülsmann M (2006): Die Desinfektion des endodontischen Systems. Endodontie 15, 147-168

Hülsmann M: Endodontie. Checklisten der Zahnmedizin. Georg Thieme Verlag, Stuttgart 2008

Hülsmann M, Stryga F (1993): Comparison of root canal preparation using different automated devices and hand instrumentation. J Endod $\underline{19}, 141-145$ Hülsmann M, Rümmelin C, Schäfers F (1997): Root canal cleanliness after preparation with different endodontic handpieces and hand instruments: a comparative SEM investigation. J Endod 23, 301-306

Hülsmann M, Heckendorff M, Schäfers F (2002): Comparative in-vitro evaluation of three chelator pastes. Int Endod J $\underline{35}, 668-679$

Hülsmann M, Gressmann G, Schäfers F (2003): A comparative study of root canal preparation using FlexMaster and HERO 642 rotary $\mathrm{Ni}$-Ti instruments. Int Endod J $\underline{36}, 358-366$

Hülsmann M, Peters OA, Dummer PMH (2005): Mechanical preparation of root canals: shaping goals, techniques and means. Endod Top $\underline{10}$, 30-76 Jiang L-M, Verhaagen B, Versluis M, van der Sluis LWM (2010): Evaluation of a Sonic Device Designed to Activate Irrigant in the Root Canal. J Endod $\underline{36}, 143-146$

Kahn FH, Rosenberg PA, Gliksberg J (1995): An in vitro evaluation of the 
irrigating characteristics of ultrasonic and subsonic handpieces and irrigating needles and probes. J Endod 21, 277-280

Khalap N, Kokate S, Hegde V (2016): Ultrasonic versus sonic activation of the final irrigant in root canals instrumented with rotary/reciprocating files: An invitro scanning electron microscopy analysis. J Conserv Dent $\underline{19}$, 368-372

Kiran S, Prakash S, Siddharth PR, Saha S, Geojan NE, Ramachandran M (2016): Comparative Evaluation of Smear Layer and Debris on the Canal Walls prepared with a Combination of Hand and Rotary ProTaper Technique using Scanning Electron Microscope. J Contemp Dent Pract 17, 574-581

Klyn SL, Kirkpatrick TC, Rutledge RE (2010): In Vitro Comparisons of Debris Removal of the EndoActivator System, the F File, Ultrasonic Irrigation, and $\mathrm{NaOCl}$ Irrigation Alone after Hand-rotary Instrumentation in Human Mandibular Molars. J Endod $\underline{36}, 1367-1371$

Leitlinie Wurzelkanalspülung s. DGZMK/DGZ Leitlinie zur Wurzelkanalspülung 2006

Littman SH (1977): Evaluation of root canal debridement by use of a radiopaque medium. J Endod $\underline{3}, 135-138$

Llena C, Cuesta C, Forner L, Mozo S, Segura J-J (2015): The effect of passive ultrasonic activation of $2 \%$ chlorhexidine or $3 \%$ sodium hypochlorite in canal wall cleaning. J Clin Exp Dent $\underline{7}, 69-73$

Lumley PJ, Walmsley AD, Walton RE, Rippin JW (1992): Effect of precurving endosonic files on the amount of debris and smear layer remaining in curved root canals. J Endod 18, 616-619

Mader CL, Baumgartner JC, Peters DD (1984): Scanning electron microscopic investigation of the smeared layer on root canal walls. J Endod $\underline{10}, 477-483$

Mancini M, Cerroni L, lorio L, Armellin E, Conte G, Cianconi L (2013): Smear Layer Removal and Canal Cleanliness Using Different Irrigation Systems (EndoActivator, EndoVac, and Passive Ultrasonic Irrigation): Field Emission Scanning Electron Microscopic Evaluation in an In Vitro Study. J Endod $\underline{39}$, 1456-1460 
Martin H (1976): Ultrasonic disinfection of the root canal. Oral Surg Oral Med Oral Pathol $\underline{42}, 92-99$

McComb D, Smith DC (1975): A preliminary scanning electron microscopic study of root canals after endodontic procedures. J Endod 1, 238-242

Meryon SD, Brook AM (1990): Penetration of dentine by three oral bacteria in vitro and their associated cytotoxicity. Int Endod J $\underline{23}, 196-202$

Moorer WR, Wesselink PR (1982): Factors promoting the tissue dissolving capability of sodium hypochlorite. Int Endod J $\underline{15}, 187-196$

Mozo S, Llena C, Chieffi N, Forner L, Ferrari M (2014): Effectiveness of passive ultrasonic irrigation in improving elimination of smear layer and opening dentinal tubules. J Clin Exp Dent $\underline{6}, 47-52$

Munoz HR, Camacho-Cuadra K (2012): In Vivo Efficacy of Three Different Endodontic Irrigation Systems for Irrigant Delivery to Working Length of Mesial Canals of Mandibular Molars. J Endod 38, 445-448

Naenni N, Thoma K, Zehnder M (2004): Soft tissue dissolution capacity of currently used and potential endodontic irrigants. J Endod $\underline{30}, 785-787$

Neuhaus KW, Liebi M, Stauffacher S, Eick S, Lussi A (2016): Antibacterial Efficacy of a New Sonic Irrigation Device for Root Canal Disinfection. J Endod 42, $1799-1803$

Niu L, Luo X, Li G, Bortoluzzi EA, Mao J, Chen J, Gutmann JL, Pashley DH, Tay FR (2014): Effects of different sonic activation protocols on debridement efficacy in teeth with single-rooted canals. J Dent $\underline{42}, 1001-1009$

Oliveira LD, Carvalho CAT, Nunes W, Valera MC, Camargo CHR, Jorge AOC (2007): Effects of chlorhexidine and sodium hypochlorite on the microhardness of root canal dentin. Oral Surg Oral Med Oral Pathol Oral Radiol Endod 104, e125-e128

Ordinola-Zapata R, Bramante CM, Aprecio RM, Handysides R, Jaramillo DE (2014): Biofilm removal by $6 \%$ sodium hypochlorite activated by different irrigation techniques. Int Endod J $\underline{47}, 659-666$

Ørstavik D, Haapasalo M (1990): Disinfection by endodontic irrigants and dressings of experimentally infected dentinal tubules. Endod Dent Traumatol 
$\underline{6}, 142-149$

Ørstavik D, Kerekes K, Molven O (1991): Effects of extensive apical reaming and calcium hydroxide dressing on bacterial infection during treatment of apical periodontitis: a pilot study. Int Endod J $\underline{24}, 1-7$

Paqué F, Balmer M, Attin T, Peters OA (2010): Preparation of oval-shaped root canals in mandibular molars using nickel-titanium rotary instruments: a micro-computed tomography study. J Endod $\underline{36}, 703-707$

Park E, Shen Y, Khakpour M, Haapasalo M (2013): Apical pressure and extent of irrigant flow beyond the needle tip during positive-pressure irrigation in an in vitro root canal model. J Endod $\underline{39}, 511-515$

Pashley DH (1992): Smear layer: overview of structure and function. Proc Finn Dent Soc $\underline{88}$, 215-224

Pashley DH, Michelich V, Kehl T (1981): Dentin permeability: effects of smear layer removal. J Prosthet Dent $\underline{46}, 531-537$

Pashley EL, Birdsong NL, Bowman K, Pashley DH (1985): Cytotoxic effects of $\mathrm{NaOCl}$ on vital tissue. J Endod 11, 525-528

Peters OA, Schönenberger K, Laib A (2001): Effects of four Ni-Ti preparation techniques on root canal geometry assessed by micro computed tomography. Int Endod J 34, 221-230

Plotino G, Pameijer CH, Maria Grande N, Somma F (2007): Ultrasonics in Endodontics: A Review of the Literature. J Endod 33, 81-95

Prado MC, Leal F, Gusman H, Simão RA, Prado M (2016): Effects of auxiliary device use on smear layer removal. J Oral Sci $\underline{58}, 561-567$

Ram Z (1977): Effectiveness of root canal irrigation. Oral Surg Oral Med Oral Pathol $\underline{44}, 306-312$

Ramamoorthi S, Nivedhitha MS, Divyanand MJ (2015): Comparative evaluation of postoperative pain after using endodontic needle and EndoActivator during root canal irrigation: A randomised controlled trial. Aust Endod J 41, 78-87

Retamozo B, Shabahang S, Johnson N, Aprecio RM, Torabinejad M (2010): Minimum contact time and concentration of sodium hypochlorite required to 
eliminate Enterococcus faecalis. J Endod $\underline{36}, 520-523$

Retsas A, Koursoumis A, Tzimpoulas N, Boutsioukis C (2016): Uncontrolled Removal of Dentin during In Vitro Ultrasonic Irrigant Activation in Curved Root Canals. J Endod 42, 1545-1549

Rico-Romano C, Zubizarreta-Macho Á, Baquero-Artigao M-R, Mena-Álvarez $J$ (2016): An analysis in vivo of intracanal bacterial load before and after chemo-mechanical preparation: A comparative analysis of two irrigants and two activation techniques. J Clin Exp Dent $\underline{8}$, e9-e13

Rödig T, Hülsmann M, Nordmeyer S, Drebenstedt S: Grundlagen der modernen Endodontie. 1. Auflage; Spitta Verlag, Balingen 2009

Rödig T, Döllmann S, Konietschke F, Drebenstedt S, Hülsmann M (2010): Effectiveness of different irrigant agitation techniques on debris and smear layer removal in curved root canals: a scanning electron microscopy study. $\mathrm{J}$ Endod $\underline{36}, 1983-1987$

Roy RA, Ahmad M, Crum LA (1994): Physical mechanisms governing the hydrodynamic response of an oscillating ultrasonic file. Int Endod J 27, 197207

Ruddle CJ (2002): Finishing the apical one third. Endodontic considerations. Dent Today 21, 66-70, 72-73

Scelza MFZ, Pierro V, Scelza P, Pereira M (2004): Effect of three different time periods of irrigation with EDTA-T, EDTA, and citric acid on smear layer removal. Oral Surg Oral Med Oral Pathol Oral Radiol Endod $\underline{98}$, 499-503

Schäfer E (2007): Irrigation of the Root Canal. Endo 1, 11-27

Schäfer E, Zapke K (2000): A comparative scanning electron microscopic investigation of the efficacy of manual and automated instrumentation of root canals. J Endod 26, 660-664

Schirrmeister JF, Wrbas K-T, Schneider FH, Altenburger MJ, Hellwig E (2006): Effectiveness of a hand file and three nickel-titanium rotary instruments for removing gutta-percha in curved root canals during retreatment. Oral Surg Oral Med Oral Pathol Oral Radiol Endod 101, 542-547 Sen BH, Safavi KE, Spångberg LS (1999): Antifungal effects of sodium 
hypochlorite and chlorhexidine in root canals. J Endod 25, 235-238

Sen BH, Ertürk O, Pişkin B (2009): The effect of different concentrations of EDTA on instrumented root canal walls. Oral Surg Oral Med Oral Pathol Oral Radiol Endod 108, 622-627

Shahravan A, Haghdoost A-A, Adl A, Rahimi H, Shadifar F (2007): Effect of smear layer on sealing ability of canal obturation: a systematic review and meta-analysis. J Endod 33, 96-105

Shovelton DS (1964): The presence and distribution of microorganisms within non vital teeth. $\mathrm{Br}$ Dent $\mathrm{J} \underline{117}, 101-107$

Singh N, Chandra A, Tikku A, Verma P (2014): A comparative evaluation of different irrigation activation systems on smear layer removal from root canal: An in-vitro scanning electron microscope study. J Conserv Dent 17, 159-163

Sirtes G, Waltimo T, Schaetzle M, Zehnder M (2005): The effects of temperature on sodium hypochlorite short-term stability, pulp dissolution capacity, and antimicrobial efficacy. J Endod 31, 669-671

Sjögren U, Figdor D, Persson S, Sundqvist G (1997): Influence of infection at the time of root filling on the outcome of endodontic treatment of teeth with apical periodontitis. Int Endod J 30, 297-306

Šnjarić D, Čarija Z, Braut A, Halaji A, Kovačević M, Kuiš D (2012): Irrigation of human prepared root canal - ex vivo based computational fluid dynamics analysis. Croat Med J $\underline{53}, 470-479$

Soares JA, Roque de Carvalho MA, Cunha Santos SM, Mendonça RMC, Ribeiro-Sobrinho AP, Brito-Júnior M, Magalhães PP, Santos MH, de Macêdo Farias L (2010): Effectiveness of chemomechanical preparation with alternating use of sodium hypochlorite and EDTA in eliminating intracanal Enterococcus faecalis biofilm. J Endod $\underline{36}, 894-898$

Spratt DA, Pratten J, Wilson M, Gulabivala K (2001): An in vitro evaluation of the antimicrobial efficacy of irrigants on biofilms of root canal isolates. Int Endod J $\underline{34}, 300-307$

Stevens RW, Strother JM, McClanahan SB (2006): Leakage and sealer penetration in smear-free dentin after a final rinse with $95 \%$ ethanol. J Endod 
$\underline{32}, 785-788$

Stojicic S, Zivkovic S, Qian W, Zhang H, Haapasalo M (2010): Tissue Dissolution by Sodium Hypochlorite: Effect of Concentration, Temperature, Agitation, and Surfactant. J Endod $\underline{36}, 1558-1562$

Subramaniam P, Girish Babu KL, Tabrez TA (2016): Effectiveness of Rotary Endodontic Instruments on Smear Layer Removal in Root Canals of Primary Teeth: A Scanning Electron Microscopy Study. J Clin Pediatr Dent 느, 141146

Tanomaru-Filho M, Franco Silveira BR, Martelo RB, Guerreiro-Tanomaru JM (2015): Influence of Concentration and Agitation of Sodium Hypochlorite and Peracetic Acid Solutions on Tissue Dissolution. J Contemp Dent Pract $\underline{16}$, 876-879

Tartari T, Oda DF, Zancan RF, da Silva TL, de Moraes IG, Duarte MH, Bramante CM (2017): Mixture of alkaline tetrasodium EDTA with sodium hypochlorite promotes in vitro smear layer removal and organic matter dissolution during biomechanical preparation. Int Endod J $\underline{50}, 106-114$

Teixeira CS, Felippe MCS, Felippe WT (2005): The effect of application time of EDTA and $\mathrm{NaOCl}$ on intracanal smear layer removal: an SEM analysis. Int Endod J $\underline{38}, 285-290$

Tewari RK, Ali S, Mishra SK, Kumar A, Andrabi SM-U-N, Zoya A, Alam S (2016): Mechanical reduction of the intracanal Enterococcus faecalis population by Hyflex CM, K3XF, ProTaper Next, and two manual instrument systems: an in vitro comparative study. J Investig Clin Dent $\underline{7}, 168-173$

Timpawat S, Vongsavan N, Messer HH (2001): Effect of removal of the smear layer on apical microleakage. J Endod 27, 351-353

Topcu KM, Karatas E, Ozsu D, Ersoy I (2014): Efficiency of the Self Adjusting File, WaveOne, Reciproc, ProTaper and hand files in root canal debridement. Eur J Dent $\underline{8}, 326-329$

Torabinejad M, Handysides R, Khademi AA, Bakland LK (2002): Clinical implications of the smear layer in endodontics: A review. Oral Surg Oral Med Oral Pathol Oral Radiol Endodontology $\underline{94}, 658-666$ 
Tronstad L, Niemczyk SP (1986): Efficacy and safety tests of six automated devices for root canal instrumentation. Dent Traumatol 2, 270-276

Turk T, Kaval ME, Şen BH (2015): Evaluation of the smear layer removal and erosive capacity of EDTA, boric acid, citric acid and desy clean solutions: an in vitro study. BMC Oral Health $\underline{15}, 1-5$

Urban K, Donnermeyer D, Schäfer E, Bürklein S (2017): Canal cleanliness using different irrigation activation systems: a SEM evaluation. Clin Oral Investig 21, 2681-2687

Uroz-Torres D, González-Rodríguez MP, Ferrer-Luque CM (2010): Effectiveness of the EndoActivator System in Removing the Smear Layer after Root Canal Instrumentation. J Endod $\underline{36}$, 308-311

Violich DR, Chandler NP (2010): The smear layer in endodontics - a review. Int Endod J $\underline{43}, 2-15$

Walters MJ, Baumgartner JC, Marshall JG (2002): Efficacy of irrigation with rotary instrumentation. J Endod 28, 837-839

Wang Y, Xiao S, Ma D, Huang X, Cai Z (2015): Minimizing Concentration of Sodium Hypochlorite in Root Canal Irrigation by Combination of Ultrasonic Irrigation with Photodynamic Treatment. Photochem Photobiol 91, 937-941

Wennberg A, Ørstavik D (1990): Adhesion of root canal sealers to bovine dentine and gutta-percha. Int Endod J 23, 13-19

Yamada RS, Armas A, Goldman M, Lin PS (1983): A scanning electron microscopic comparison of a high volume final flush with several irrigating solutions: Part 3. J Endod 9, 137-142

Yamaguchi M, Yoshida K, Suzuki R, Nakamura H (1996): Root canal irrigation with citric acid solution. J Endod 22, 27-29

Yeung W, Raldi DP, Cunha RS, Mello I (2014): Assessment of smear layer removal protocols in curved root canals. Aust Endod J $\underline{40}, 66-71$

Yost RA, Bergeron BE, Kirkpatrick TC, Roberts MD, Roberts HW, Himel VT, Sabey KA (2015): Evaluation of 4 Different Irrigating Systems for Apical Extrusion of Sodium Hypochlorite. J Endod 41, 1530-1534

Zaparolli D, Saquy PC, Cruz-Filho AM (2012): Effect of sodium hypochlorite 
and EDTA irrigation, individually and in alternation, on dentin microhardness at the furcation area of mandibular molars. Braz Dent J $\underline{23}, 654-658$

Zehnder M, Schmidlin P, Sener B, Waltimo T (2005): Chelation in root canal therapy reconsidered. J Endod $\underline{31}, 817-820$ 
Danksagung

Herzlichst danke ich Herrn Prof. Dr. M. Hülsmann für die freundliche Überlassung des Themas, die vielen Anregungen und Hinweise, die stets konstruktive Kritik sowie die immer sehr zeitnahe Korrektur und stete Motivation.

Herzlichen Dank an Frau Carola Hartje für den stets schnellen Nachschub an Materialien sowie Geräten.

Herrn Hans-Georg Sydow aus dem Zentrum Anatomie danke ich für die gute und sehr freundliche Betreuung während der Bedampfung der Proben.

Frau Xenia Schulz aus der Medizinischen Statistik danke ich für die geduldige und freundliche Betreuung bei der statistischen Auswertung. 
